\title{
Synchronization of Coupled Chemical Oscillators and Collective Behavior of Self-Propelled Particles
}

\author{
Desmond Tutu Soti Yengi
}

Follow this and additional works at: https://researchrepository.wvu.edu/etd

\section{Recommended Citation}

Yengi, Desmond Tutu Soti, "Synchronization of Coupled Chemical Oscillators and Collective Behavior of Self-Propelled Particles" (2017). Graduate Theses, Dissertations, and Problem Reports. 7284.

https://researchrepository.wvu.edu/etd/7284

This Dissertation is protected by copyright and/or related rights. It has been brought to you by the The Research Repository @ WVU with permission from the rights-holder(s). You are free to use this Dissertation in any way that is permitted by the copyright and related rights legislation that applies to your use. For other uses you must obtain permission from the rights-holder(s) directly, unless additional rights are indicated by a Creative Commons license in the record and/ or on the work itself. This Dissertation has been accepted for inclusion in WVU Graduate Theses, Dissertations, and Problem Reports collection by an authorized administrator of The Research Repository @ WVU.

For more information, please contact researchrepository@mail.wvu.edu. 


\title{
Synchronization of Coupled Chemical Oscillators and Collective Behavior of Self-Propelled Particles
}

\author{
Desmond Tutu Soti Yengi \\ Dissertation submitted \\ to the Eberly College of Arts and Sciences \\ at West Virginia University
}

in partial fulfillment of the requirements for the degree of

Doctor of Philosophy in

Chemistry

Kenneth Showalter, Ph.D., Chair

Charles Jaffé, Ph.D.

Terry Gullion, Ph.D.

Justin Legleiter, Ph.D.

Adam Halasz, Ph.D.

Mark Tinsley, Ph.D.

C. Eugene Bennett Department of Chemistry

Morgantown, West Virginia

2017

Keywords: Photosensitive BZ reaction, chemical oscillators, phase response curves, synchronization, collective behaviors, autonomous switching pattern, excitatory and inhibitory coupling, self-propelled janus particles

Copyright 2017 Desmond Tutu Soti Yengi 


\section{Abstract \\ Synchronization of Coupled Chemical Oscillators and Collective Behavior of Self-Propelled Particles}

\section{Desmond Tutu Soti Yengi}

We experimentally and computationally investigate the collective behavior of coupled chemical oscillators and non-biological self-propelled particles (SPP). To characterize the collective behavior, we must understand how the individual chemical oscillators respond to stimuli. These oscillators have been shown in previous studies to self-organize through quorum sensing and be influenced by light. The sensitivity of the oscillators to light stimuli can be characterized by their phase response curves (PRC). The PRC of the Belousov-Zhabotinsky (BZ) oscillator quantifies the excitatory-inhibitory degree of the BZ reaction system. By using catalytic $\mathrm{Ru}(\mathrm{bpy})_{3}^{2+}$-loaded cation exchange beads in catalyst-free BZ solutions, we realize various network topologies by coupling these beads via their light sensitivity at $440-460 \mathrm{~nm}$. We characterize the PRC of the BZ oscillator and the dynamical behavior in networks of heterogeneous BZ oscillators with varying degrees of excitatory-inhibitory dynamics. Phase diagrams of heterogeneity versus excitatory-inhibitory dynamics and of heterogeneity versus coupling strength show regions of in-phase, out-of-phase, bistable, and autonomous switching patterns.

Inspired by the collective motions found in the schooling of fish, flocking of birds, and swarming of insects, we also examine the dynamical behavior of agglomerates of micrometer sized platinum-silica particles in solutions of hydrogen peroxide. The motion of platinum-silica particles ( $1 \mu \mathrm{m}$ in size) dispersed in hydrogen peroxide solutions occurs due to chemically produced concentration gradients (diffusiophoresis). We quantitatively demonstrate how the orientations of the particles in the agglomerates affect the resultant motion pattern. This work has a potential to enable the control of motion in SPP agglomerates by varying the orientation of the particles. 


\title{
Dedication
}

\author{
To my families
}

Rojo and Soti family especially my grandmother, Raele Rojo, and mother, Mary Yangi

Anne Perkins and Mark Everall family

Joanna Showcross and Charles Peck family

Linda and Bob Hansen family

I am grateful for your love, patience, encouragement, and support.

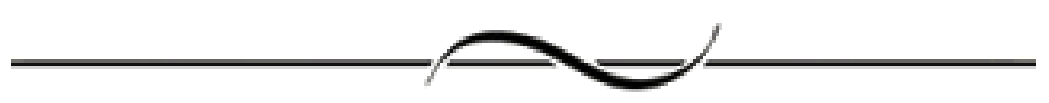

In memory of Dani Stephen Woja 


\section{Acknowledgments}

This work has indeed been a labor of love and a test of endurance. In this journey prior to and during the graduate program, I have been fortified and encouraged by countless individuals who I want to recognize here. Thank you for helping in opening doors for me into many possibilities. I am fortunate to have encountered you on this journey.

First, I would like to acknowledge the guidance of my research advisor Dr. Kenneth Showalter who gave me the incalculable benefit of his experience and wisdom. Thank you for being supportive and patiently reading the various drafts and providing valuable comments. Special thanks also go to Dr. Mark Tinsley for giving me insights, pointers, and suggestions throughout my research projects. I like to also thank my undergraduate research mentor Dr. Glen Frerichs in Westminster College, MO for getting me interested in the world of nonlinear dynamics. I am grateful to my Ph.D. committee members Dr. Terry Gullion, Dr. Charles Jaffé, Dr. Justin Legleiter, and Dr. Adam Halasz for taking the time in reviewing and evaluating my dissertation.

I want to take the opportunity to thank Dr. Ayusman Sen of Penn State University for providing us with the Janus particles that we used in the self-propelled particle project. Dr. Tatsunari Sakurai of Chiba University, Tokyo, Japan, Jan Totz and Winnie Poel of Technical University of Berlin, Germany, and Jirapa Rueangsuwan from Mahidol University, Bangkok, Thailand, thank you all for collaborating on some of the projects. To former and current Showaltees Dr. Calistus Ngonghala, Dr. Simbarashe Nkomo, Dr. Hua Ke, Dr. Darrell Collison, and Dr. Razan Sanari, Tianran Chen; Sadegh Ganjabad, Syed Jazli Syed Jamaluddin, and David Mersing, thank you for providing the supportive and friendly environment. I am grateful to Rebecca Secrist for being patient dealing with me in several of my paperwork. Thanks for the kindness and care. I would also like to recognize the help of other staff, to mention few Brenda Prentiss, Sherman Adams, Allen Burns, and Randall Eaglen.

I owe a special debt to the Shelby Davis Foundation for the generous scholarship; I wouldn't have been in a position to attend college without the scholarship. I am also grateful to Senator Angus King's office for taking my visa application case to inquire on my behalf in 2014- 
2015. To my high school, United World College of Atlantic, thank you! I hope I will get to be able to pay it forward.

I want to thank Syed Jazli Syed Jamaluddin, Henry Peck, Fraser Harland, and Claire KbHarland for patiently reading through at least one chapter of my first drafts of this dissertation and providing valuable feedbacks. I am so grateful to Michael Modi, Robert Andruga, Charles Bafaki, Martha Hewison, Gareth Rees, and Dr. Patrick Kirby.

I couldn't have asked for more solid and supportive friends and family members. I will not be able to mention you all here individually but will mention few, especially Precious Simalundu, Emmi Antinoja, Chelsea Hodgkins, Henry Peck, Fraser Harland, David Lao Yifeng, Jimmy Longun, George Sworo, Kennedy Maring, Michael Dusman, Moses Dani, Francis Yengistic, Patrick Amanzuru, and Noel Bejur, thank you for the support and encouragements. 


\section{Table of Contents}

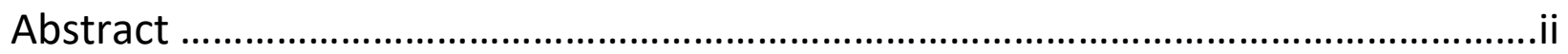

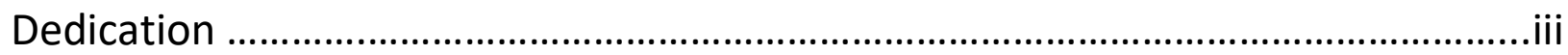

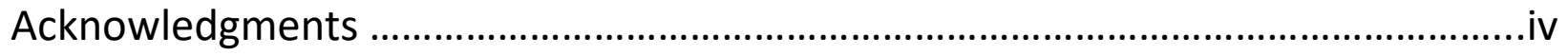

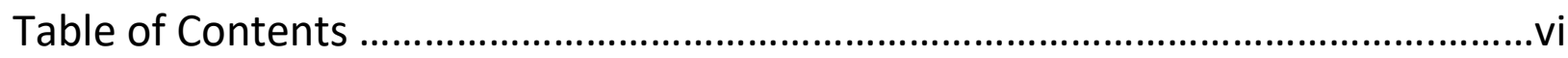

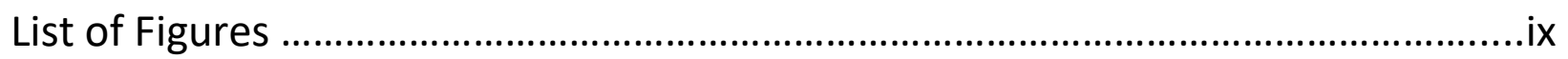

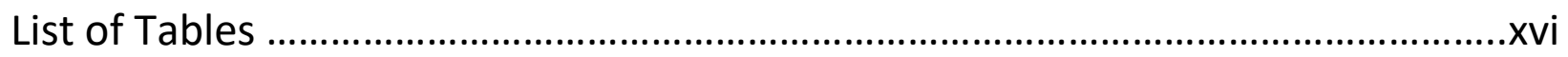

1 Synchronization of Biological and Chemical Oscillators. ........................1

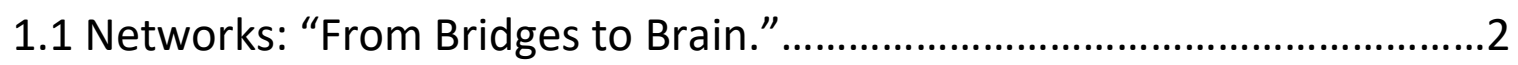

1.2 Network in the Kuramoto Model. .........................................................

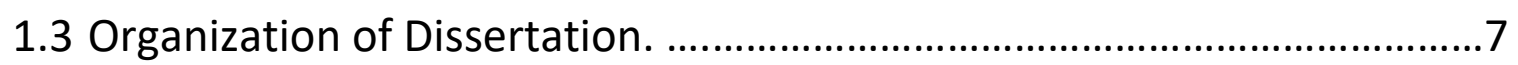

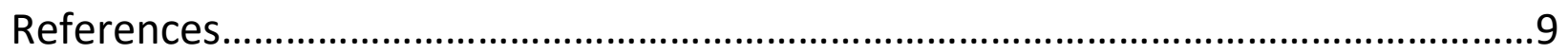

2 The Belousov-Zhabotinsky (BZ) Reaction. .............................................................12

2.1 Background/Introduction. ................................................................... 12

2.2.1 The BZ Reaction Mechanism. ....................................................13

2.2.2 The Oregonator Model. .............................................................. 15

2.3.1 ZBKE Reaction Mechanism. .......................................................18

2.3.2 Kinetic Model: ZBKE Model. ..................................................19

2.4.1 Effect of Light on the BZ Reaction. ...........................................23

2.4.2 The Photo-Chemical Mechanism. ..............................................23

2.4.3 Light sensitive ZBKE model modification. ..................................24

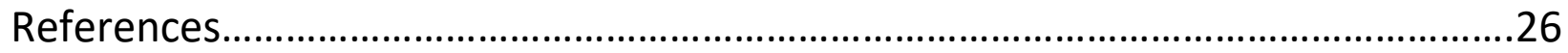


3 Discretization and Coupling. .................................................................................. 28

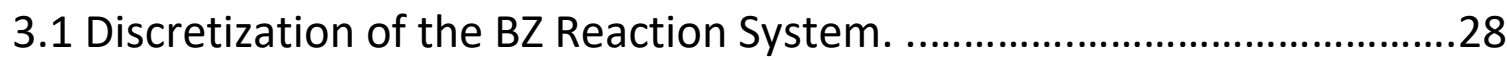

3.2 Photochemical Coupling of BZ Oscillators in a Network. ........................29

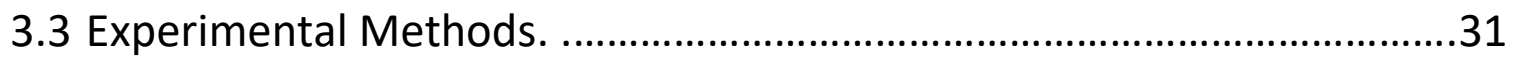

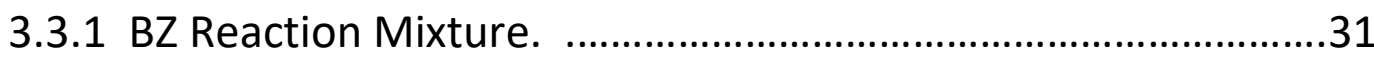

3.3.2 Ruthenium (II) Loaded Oscillator Beads Preparation. ................31

3.3.3 The Oscillator System Preparation. …..........................................31

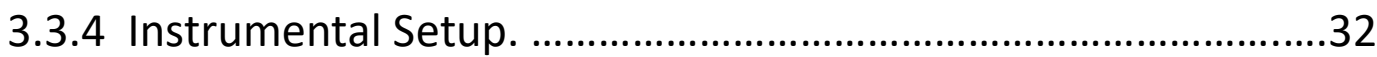

3.3.5 Computer-Controlled and Photochemical Coupling. .................33

3.4 Numerical Simulations: Coupled ZBKE Oscillator Model. ........................35

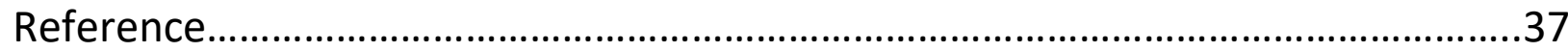

4 Coupled BZ Chemical Oscillators. ...........................................................................40

4.1 Phase Response Curve: Photosensitive $\mathrm{Ru}(\mathrm{bpy})_{3}^{2+}$-Catalyzed

BZ Oscillator.

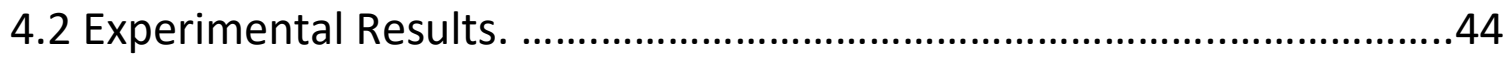

4.2.1 Phase Response Curve and Phase Transition Curve. .................44

4.2.1.1 The Effect of Bromate Concentration in the BZ Reaction Mixture. ..........................................................44

4.2.1.2 The Effect of Bromomalonic Acid Concentration in the BZ Reaction Mixture. ............................................46

4.3 PRC Numerical Simulations Results. ........................................................48

4.4 Coupling Pair of BZ Oscillators. .............................................49 
4.4.1 Phase-Locked Synchronization. .................................................51

4.4.2 Autonomously Switching Pattern. ..........................................53

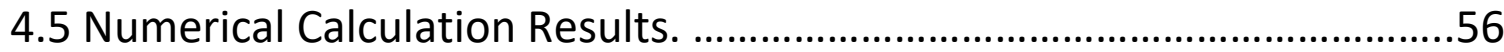

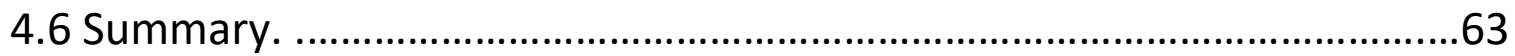

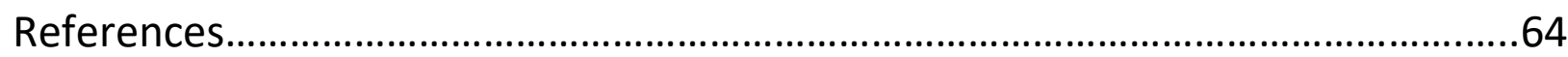

5 Collective Behavior of Self-Propelled Particles. .................................................66

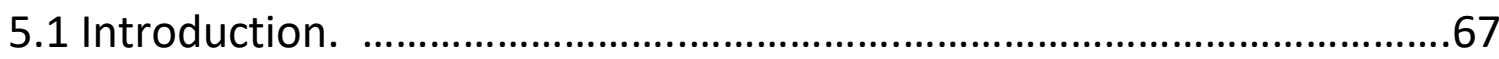

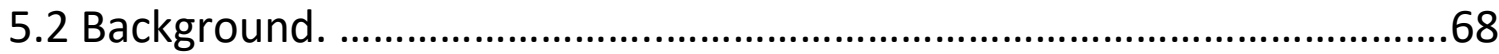

5.2.1 Solving the Challenges: Overcoming Brownian Motion in Microscale Particles. ................................................................69

5.2.1.1 Reaction-Driven Propulsion. .........................................70

5.2.1.2 Propulsion by Broken Symmetry. ...................................70

5.2.2 Hydrogen Peroxide Fueled Platinum-Silica Janus

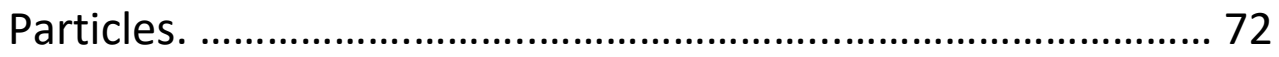

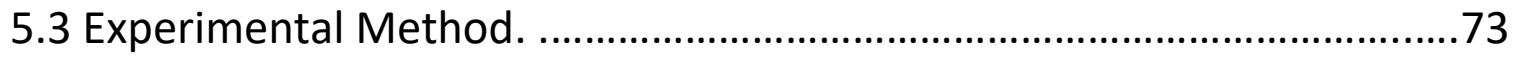

5.3.1 Platinum-Silica Particles Fabrication. ........................................73

5.3.2 Experimental Procedure. .........................................................73

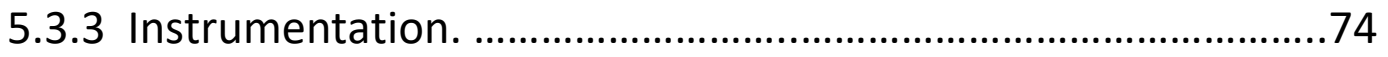

5.4 Analysis of the motion of self-propelled particles. ...................................75

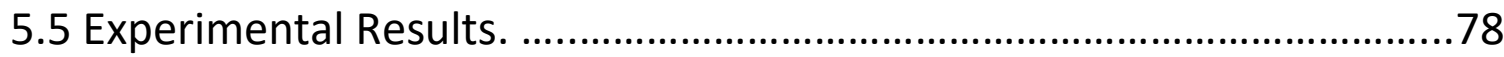

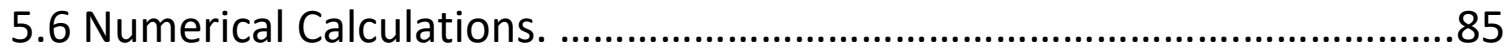

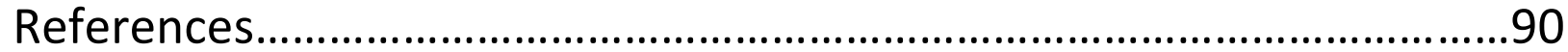




\section{List of Figures}

Figure 1.1: Map showing Königsberg with layout of the seven bridges in Euler's time from Bogdan Giuşcă $[16,17]$.

Figure 1.2: Representation of Königsberg bridges with river banks and the Island represented with nodes and the bridges connecting them represented by links. 2

Figure 1.3: Illustrations of networks. (a) A statistical model of the network of connections between brain regions [19]. (b) Friendship network with nodes representing people and links representing friendships. Colors indicate friendship clusters (from Matt Baggott) [18]. (c) Internet network with nodes representing computers and links representing wired or wireless connections between computers [20]. 3

Figure 1.4: Illustration of global, all-to-all, ring, linear, mesh, tree, and star network topologies [24].

Figure 1.5: Phase plots showing a collection of phase oscillators with an average phase, $\psi$, in the unit circle. The order parameter, $r$, measures the degree of phase synchronization. . .5

Figure 1.6: Synchronization diagram showing the Kuramoto order parameter, $r$, as a function of $\mathrm{K}$. Typical transition curve from asynchronization to synchronization with the Kuramoto model. The critical coupling strength $K_{c} \approx 1.0 \times 10^{-3}$ for the distribution $g(\omega)$................................6

Figure 1.7: Phase plots showing a collection of oscillators in the unit circle, where the color of each oscillator represents its natural period. As coupling strength $\mathrm{K}$ increases, oscillators undergo synchronization.

Figure 2.1: Spatiotemporal pattern formation in the ferroin-catalyzed BZ reaction. The pictures were taken at time intervals of 6 hours from (a) - (c). (Outside the ring the image is reflected by the container walls.) .14

Figure 2.2: Time series showing oscillation for the oxidized catalyst $(\mathrm{Z})$, bromous acid $\left(\mathrm{HBrO}_{2}\right)$ $(X)$, and bromide ion $(Y)$ from Oregonator model simulations of the BZ reaction. The concentrations were calculated by the numerical integration of the differential equations Eq. (2.6) with the rate constants $\mathrm{k}_{1}=6.28, \mathrm{k}_{2}=2.4 \times 10^{6}, \mathrm{k}_{3}=3.0, \mathrm{k}_{4}=240.0, \mathrm{k}_{\mathrm{c}}=1.0$, $h=1.0$, and initial concentrations of bromate $(A)=0.4$ and malonic acid $(B)=0.12$.....16 
Figure 2.3: Plot of $Z$ vs. $X$ and $Z$ vs. $Y$ showing the limit cycle of the $B Z$ reaction from the Oregonator model simulation.

Figure 2.4: Time series showing the oscillations of the oxidized form of the catalyst $(Z)$ and bromous acid (X) from ZBKE model simulations of the BZ reaction system.

Figure 2.5: A plot of $Z$ vs. $X$ and $Z$ vs. $Y$ showing the limit cycle of the $B Z$ reaction from $Z B K E$ model simulations. .22

Figure 3.1: Network of five coupled oscillators. 30

Figure 3.2: Catalytic $\mathrm{Ru}(\mathrm{bpy})_{3}{ }^{2+}-$ loaded cation exchange beads (200-250 microns in diameter) in catalyst-free Belousov-Zabotinsky (BZ) solutions.

Figure 3.3: Schematic diagram of the experimental setup for the BZ reaction system with discrete Ru-catalyst loaded beads [18]. .33

Figure 3.4: Time series showing grayscale values of Ru-catalyst loaded bead. .35

Figure 4.1: Time series plots showing phase shifts (phase advancing and phase delaying). (a) After perturbation, the period of oscillation decreases, and (b) after perturbation, the period of oscillation increases. The solid blue line shows the oscillations with the natural period, while the dotted blue line shows the course of oscillations after a perturbation at the point shown by the vertical arrow after time $t$ from the previous oscillation peak.

Figure 4.2: Phase response curves for the BZ reaction that show the transition from inhibitory to excitatory regimes as bromate concentration is increased. Experimental PRCs (left) and PTCS (corresponding right) of $\mathrm{Ru}(\mathrm{bpy})_{3}^{2+}$-catalyzed $\mathrm{BZ}$ oscillators. (a) $\left[\mathrm{BrO}_{3}^{-}\right]=0.29 \mathrm{M}$ (b) $\left[\mathrm{BrO}_{3}^{-}\right]=$ $0.41 \mathrm{M}(\mathrm{c})\left[\mathrm{BrO}_{3}^{-}\right]=0.61 \mathrm{M}$.

Figure 4.3: PRC and PTC plots that show the transition from inhibitory to excitatory regimes as bromide concentration is decreased. Experimental PRCs (left) and PTCs (corresponding right) of $\mathrm{Ru}(\mathrm{bpy})_{3}^{2+}$-catalyzed BZ oscillators. (a) $[\mathrm{BrMA}]=0.096 \mathrm{M},[\mathrm{MA}]=0.000 \mathrm{M},\left[\mathrm{Br}^{-}\right]=0.026$ (b) $[\mathrm{BrMA}]=0.096 \mathrm{M},[\mathrm{MA}]=0.000 \mathrm{M}$ (c) $[\mathrm{BrMA}]=0.03 \mathrm{M},[\mathrm{MA}]=0.066 \mathrm{M}$. 
Figure 4.4: Simulations of the PRC and PTC using the modified ZBKE model. The plots show the transition from the inhibitory regime to excitatory regime as $\alpha$ increases. The plots on the left are the PRCs and the plots on the right are the PTCs described by Eqs. (4.1)-(4.3). (a) $\alpha_{0}=0.10$ (b) $\alpha_{0}=0.50$ (c) $\alpha_{0}=0.70$. 48

Figure 4.5: Time series (blue line) showing oscillations of interacting oscillators (1) and (2). The interaction signals are shown by the dotted red line. .50

Figure 4.6: PRC and PTC plots for coupled BZ oscillators with competing inhibitory and excitatory regimes. .50

Figure 4.7: A pair of coupled Ru-catalyzed BZ oscillators, displaying anti-phase synchronization: (a) time series, (b) calculated periods, and (c) perturbation phases. Coupling is turned on after 500 seconds and turned off close to 3000 seconds; the experiment runs for approximately one hour. Coupling is applied between the green lines. Initial concentrations: $[\mathrm{MA}]=0.006 \mathrm{M},[\mathrm{BrMA}]=0.09 \mathrm{M},\left[\mathrm{BrO}_{3}^{-}\right]=0.45 \mathrm{M}$, and $\left[\mathrm{H}_{2} \mathrm{SO}_{4}\right] \approx 0.78 \mathrm{M}$. .52

Figure 4.8: A pair of coupled Ru-catalyzed BZ oscillators displaying in-phase synchronization: (a) time series, (b) calculated periods, and (c) perturbation phases. Coupling is applied between the green lines. Initial concentrations: $[\mathrm{MA}]=0.006 \mathrm{M},[\mathrm{BrMA}]=0.09 \mathrm{M},\left[\mathrm{BrO}_{3}^{-}\right]=0.45 \mathrm{M}$, and $\left[\mathrm{H}_{2} \mathrm{SO}_{4}\right] \approx 0.78 \mathrm{M}$.

Figure 4.9: A pair of coupled Ru-catalyzed BZ oscillators displaying switching patterns from inphase to out-of-phase synchronization: (a) time series, (b) calculated periods, and (c) perturbation phases. Coupling is applied between the green lines. Initial concentrations: $[\mathrm{MA}]=0.006 \mathrm{M},[\mathrm{BrMA}]=0.09 \mathrm{M},\left[\mathrm{BrO}_{3}^{-}\right]=0.45 \mathrm{M}$, and $\left[\mathrm{H}_{2} \mathrm{SO}_{4}\right] \approx 0.78 \mathrm{M}$. .54

Figure 4.10: A pair of coupled Ru-catalyzed BZ oscillators displaying switching patterns from out-of-phase to in-phase synchronization: (a) time series, (b) calculated periods, and (c) perturbation phases. Coupling is applied between the green lines. Initial concentrations: $[\mathrm{MA}]=0.006 \mathrm{M},[\mathrm{BrMA}]=0.09 \mathrm{M},\left[\mathrm{BrO}_{3}^{-}\right]=0.45 \mathrm{M}$, and $\left[\mathrm{H}_{2} \mathrm{SO}_{4}\right] \approx 0.78 \mathrm{M}$. .55 
Figure 4.11: A pair of coupled Ru-catalyzed BZ oscillators displaying switching synchronization: (a) time series, (b) calculated periods, and (c) perturbation phases. Coupling is applied between the green lines. Initial concentrations: $[\mathrm{MA}]=0.006 \mathrm{M},[\mathrm{BrMA}]=0.09 \mathrm{M},\left[\mathrm{BrO}_{3}^{-}\right]=0.45 \mathrm{M}$, and $\left[\mathrm{H}_{2} \mathrm{SO}_{4}\right] \approx 0.78 \mathrm{M}$.

Figure 4.12: A pair of coupled oscillators displaying switching synchronization: (a) time series, (b) calculated periods, and (c) perturbation phases. Coupling is applied between the green lines. See section 3.3.1 for the modified ZBKE model with general parameters, additional specific parameters are: $\alpha_{0}=0.45, \mathrm{q}_{1}=0.97284, \mathrm{q}_{2}=0.74387$, and $\mathrm{k}=2.53 \times 10^{-3}$. 57

Figure 4.13: Switching synchronization patterns. Coupling is applied between the green lines. See section 3.3.1 for the modified ZBKE model with general parameters, additional specific parameters are: $\alpha_{0}=0.45$, (a) $\mathrm{q}_{1}=0.6673, \mathrm{q}_{2}=0.5426\left(\mathrm{~T}_{1}=33.1, \mathrm{~T}_{2}=30.4\right)$, (b) $\mathrm{q}_{1}=0.6528$, $\mathrm{q}_{2}=0.5598\left(\mathrm{~T}_{1}=32.8, \mathrm{~T}_{2}=30.8\right),(\mathrm{c}) \mathrm{q}_{1}=0.5875, \mathrm{q}_{2}=0.5026\left(\mathrm{~T}_{1}=31.4, \mathrm{~T}_{2}=29.6\right)$, and (d) $\mathrm{q}_{1}=0.6673, \mathrm{q}_{2}=0.5526\left(\mathrm{~T}_{1}=33.1, \mathrm{~T}_{2}=30.7\right)$. .58

Figure 4.14: Period $(n+1)$ as a function of period $(n)$ for the switching synchronized patterns. The parameters for panels in Figure 4.14 correspond to the parameters for panels in Figure 4.13 .

Figure 4.15: Iterative phase map (PRC and PTC). The perturbation phase of the light pulse from the coupled oscillators' interaction. The PRC shows shifting up or down according to whether the oscillators are being phase advanced or phase delayed. The parameters for panels in Figure 4.15 correspond to the parameters for panels in Figure 4.13 . .60

Figure 4.16: Simulations with a modified ZBKE model of heterogeneous, photosensitive BZ oscillators. The synchronization patterns with in-phase (dark blue), out-of-phase (green) synchronization patterns, bistable synchronizations (grey), and switching patterns (red) as a function of the heterogeneous oscillator period distribution and the inhibitory-excitatory regimes weight. See section 3.3.1 for the modified ZBKE model with general parameters; additional specific parameters are: $\alpha_{0}=\left[\begin{array}{ll}0.0 & 1.0\end{array}\right], q_{1}=0.8580, q_{2}=\left[\begin{array}{lll}0.8543 & 0.6993\end{array}\right]$, $k=2.53 \times 10^{-3}$ 61 
Figure 4.17: Simulations with a modified ZBKE model of heterogeneous, photosensitive BZ oscillators. The synchronization patterns with in-phase (dark blue), out-of-phase (light blue) synchronization patterns, and switching patterns (other colors) as a function of the oscillator period distribution and coupling strength, $k$, in three different inhibitory-excitatory regimes weight, $\alpha_{0}$. See section 3.3.1 for the modified ZBKE model with general parameters; additional specific parameters are: $q_{1}=0.8580, q_{2}=\left[\begin{array}{ll}0.85430 .6993\end{array}\right]$. 62

Figure 5.1: E. coli Bacterium. From Marshall Brain, HowStuffWorks.com. .69

Figure 5.2: A schematic representation of a spherical particle of radius $R$ with the enzymatic/catalytic site on the surface. Figure is from Ref. [3]. .71

Figure 5.3: Schematic representation of Janus $\mathrm{Pt}_{-} \mathrm{SiO}_{2}$ particle undergoing diffusiophoresis. ....72

Figure 5.4: A schematic illustration of the preparation of Janus particles. .73

Figure 5.5: Experimental setup. .74

Figure 5.6: Trajectories of $\mathrm{Pt}_{-} \mathrm{SiO}_{2}$ and control pure silica particles at different hydrogen peroxide concentrations. Figure adapted from Ref. [4]. .75

Figure 5.7: Plot of mean-square displacement $\left(\Delta \mathrm{L}^{2}\right)$ as a function of time $(\Delta \mathrm{t})$ of trajectories in Figure 5.6. Figure adapted from Ref. [8]. .76

Figure 5.8: Experimental images showing $\mathrm{Pt}_{-} \mathrm{SiO}_{2}$ particles agglomerates. . .78

Figure 5.9: Illustration of $\mathrm{Pt}_{-} \mathrm{SiO}_{2}$ particle agglomerates, (a) dimers, (b) trimers, and (c) tetramers, showing some possible relative particle orientations. Blue and white represent platinum and silica surfaces, respectively. .79

Figure 5.10: Motion of half-coated Pt-silica trimer in $5.0 \% \mathrm{w} / \mathrm{w}$ hydrogen peroxide solution. The orientations of the Pt-silica particles are shown in the insert of (a). The image sequences were collected at $10.40 \mathrm{fps}$. a) The trajectory of trimer and b) mean squared displacement as a function of time steps (blue line) fitted by Eq. 7. At short times, $\Delta t<\tau_{R}$, Eq. 8 is fitted by the parabola shown by the green line, and for long times, $\Delta t>\tau_{R}$, a straight line is fitted by Eq.9 
(red line). The fits yield the characteristic quantities, $v=2.10 \mu \mathrm{m} \mathrm{s}^{-1}, D_{t}=0.221 \mu \mathrm{m}^{2} \mathrm{~s}^{-1}$, and $\tau_{R}=3.66 \mathrm{~s}$. .80

Figure 5.11: (a) Distribution of the step length $\Delta$ for trimer Pt-silica particles. (b) Effective diffusion coefficient $D_{e f f}$ as a function of the observation time interval, $\Delta t$. .81

Figure 5.12: Circular trajectory and rotation motion of half-coated Pt-silica trimer in 5.0\% w/w hydrogen peroxide solution. The orientations of the Pt-silica particles are shown in the insert at (a).The image sequences were collected at $10.40 \mathrm{fps}$. (a) The trajectory of a trimer and (b) mean square displacement. 82

Figure 5.13: (a) Distribution of the step length $\Delta$ for trimer Pt-silica particles. (b) The time variation of $\Theta(t)$ in plots of cumulative rotational angle over time. .82

Figure 5.14: Plots of (a) mean-squared angular displacement fitted with Eq. 14 and (b) mean square displacement measurements with analytical fit of Eq. 13. .83

Figure 5.15: Spiral motion of half-coated Pt-silica trimer in $22.5 \% \mathrm{w} / \mathrm{w}$ hydrogen peroxide solution. The orientations of the Pt-silica particles are shown in the insert at (a). The image sequences were collected at $10.40 \mathrm{fps}$. (a) The trajectory of the trimer and (b) the mean square displacement. .84

Figure 5.16: (a) Plots of cumulative rotational angle over time and (b) mean square angular displacement. .84

Figure 5.17: (a) Distribution of the step length, $\Delta$, for trimer Pt-silica particles. (b) Mean square displacement measurements with fit using Eq. 13. .84

Figure 5.18: Schematic representation of Janus particle agglomerates with multiple catalytic sites oriented in different directions. . .86

Figure 5.19: Active trimer trajectory with simulation parameters $\varphi_{1}=60^{\circ}, \varphi_{2}=240^{\circ}, \varphi_{3}=90^{\circ}$, $D_{t}=0.22, D_{r}=0.4, \mathrm{v}=2.10$, and $\omega_{s} \leq \omega=0.5$. (a) - (b) The trajectories of the trimer and (c) mean square displacements. .86 
Figure 5.20: Active trimer trajectory with simulation parameters $\varphi_{1}=100^{\circ}, \varphi_{2}=80^{\circ}, \varphi_{3}=60^{\circ}$, $D_{t}=0.385, D_{r}=0.0432, \mathrm{v}=8.0, \omega_{s}=0.36$, and $\omega=5.0$. (a) The trajectory of the trimer and (b) mean square displacement.

Figure 5.21: Active trimer trajectory with simulation parameters $\varphi_{1}=150^{\circ}, \varphi_{2}=100^{\circ}, \varphi_{3}=$ $260^{\circ}, D_{t}=0.35, D_{r}=0.8565, \mathrm{v}=9.5, \omega_{s}=1.56$, and $\omega=4.75$. (a) The trajectory of the trimer and (b) mean square displacement.

Figure 5.22: Illustration of $\mathrm{Pt}-\mathrm{SiO}_{2}$ particle agglomerates with a possible resulting motion for (a) dimers, (b) trimers, and (c) tetramers. 


\section{List of Tables}

Table 1.1: Table showing different systems that can be represented as a network.

Table 2.1: Table shows the ZBKE mechanism, describing the simplified reaction scheme for the bromate-catalyst-bromomalonic acid system.

Table 3.1: Initial concentrations for the catalyst-free BZ solution and catalyst-loaded oscillator beads.

Table 5.1: Values of $\tau_{R}, D_{r}, v, D_{t}$, and $D_{e f f}$ from MSD and step length probability density distribution ( $\Delta$ Distribution) measurements.

Table 5.2: Values of $\omega, \tau_{R}, D_{r}, v$, and $D_{t}$ from mean-square displacement fit and meansquare angular displacement measurements.

Table 5.3: Values of $\omega, \tau_{R}, D_{r}, v$, and $D_{t}$ from mean-square displacement fit and mean-square angular displacement measurements. 


\section{Chapter One}

\subsection{Synchronization of Biological and Chemical Oscillators}

The Belousov-Zhabotinsky (BZ) reaction exhibits a rich variety of dynamical behaviors that are similar to behaviors found in biological systems. Examples include fireflies flashing in unison [1, 2], interaction of neurons in the brain $[3,4,5]$, and central pattern generators that control rhythmic actions [6]. The dynamical behavior of the oscillators involved in these systems shows remarkable selforganization as a result of their interactions. The fundamental principles that govern the dynamics of these oscillators are the same. For example, interacting oscillatory systems, such as yeast cells or BZ oscillators, tend to exhibit a collective rhythm. These interactions occur by mutual signaling in the form of stimuli that alter the behavior of the oscillators. Oscillatory self-organization in biological systems is essential for controlling the timing in many processes in living systems $[7,8]$.

These oscillators also share essential features such as nonlinearity, heterogeneity, and an excitatory-inhibitory response to stimuli. In the excitatory-inhibitory regimes, the excitatory signal is interlinked with the inhibitory signal to varying degrees [9]. The excitatory-inhibitory regulatory feature in an oscillator signaling is common in biological oscillators such as the circadian clock $[7,8,10]$ and cell cycle $[11,12]$. The nervous system also relies on excitatory and inhibitory signaling. For example, cognition involves a balance of excitation and inhibition neurotransmitters to maintain a certain pattern of activity [9]. Autism [9, 13] and epileptic seizure are conditions where the ratio of excitatory to inhibitory neurotransmitters increases beyond a normal range, giving rise to enhanced synchronization activity in the neurons [9, 14]. On the other hand, a condition like schizophrenia, with reduced neural activity, has a relatively low ratio of excitatory to inhibitory neurotransmitters. Glutamate and GABA (gammaaminobutyric acid) are chemical neurotransmitters found in brain synapses, which are associated with excitation and inhibition, respectively $[9,13,15]$.

Similarly, the BZ reaction consists of excitatory and inhibitory reaction channels, and the reaction mixture composition can be varied to produce a particular degree of excitatoryinhibitory behavior. Depending on the extent of the excitatory-inhibitory behavior and the heterogeneity of the coupled BZ oscillators, the oscillatory behavior can range from simple synchronization to complex patterns of oscillation. 


\subsection{Networks: "From Bridges to Brain."}

Dynamical behaviors can be often better understood using network theory, which began in the $18^{\text {th }}$ century in Königsberg, the ancient Prussian city now Kaliningrad, when Leonhard Euler solved the Bridges of Königsberg problem $[16,17]$. The city of Königsberg is divided into four land masses by the branches of Pregel River. The island and river banks are connected by seven bridges, as shown in Figure 1.1.

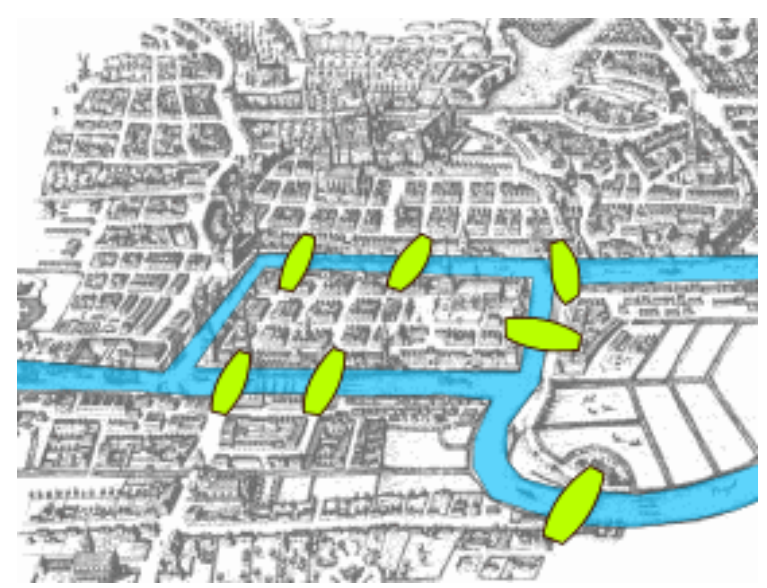

Figure 1.1: Map showing Königsberg with layout of the seven bridges in Euler's time from Bogdan Giuşcă $[16,17]$.

The Königsberg bridges problem was to determine whether it is possible to walk a path starting and ending on the same land mass by crossing each bridge exactly once. Euler thought about the different land masses in Königsberg as nodes linked by lines representing the different bridges, as illustrated in Figure 1.2. The Euler approach in solving the problem had a profound effect on the development of network theory.

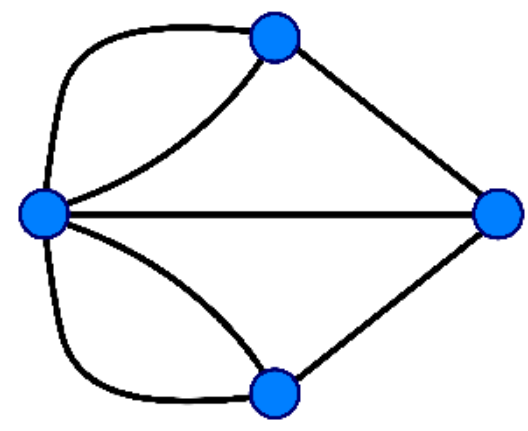

Figure 1.2: Representation of Königsberg bridges with river Banks and the Island represented with nodes and the bridges connecting them represented by links. 
The network consists of nodes connected by links. Nodes represent the individual elements in the network, and the links determine the interaction mechanism of the elements. Networks are found in systems such as ecologies, economies, societies, biological systems, and chemical systems. For example, in the brain action potential signals are transmitted via synapses, and in the internet network, binary digits are transmitted via wires or wirelessly.

(a)

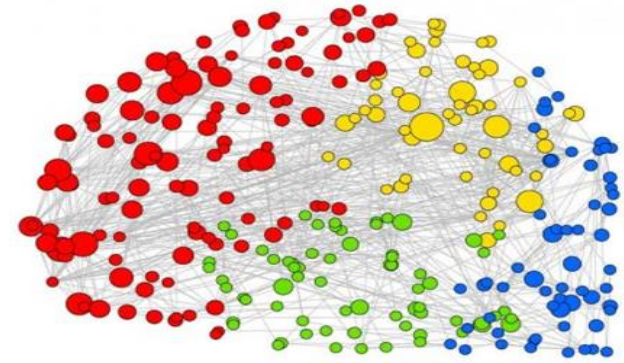

(b)

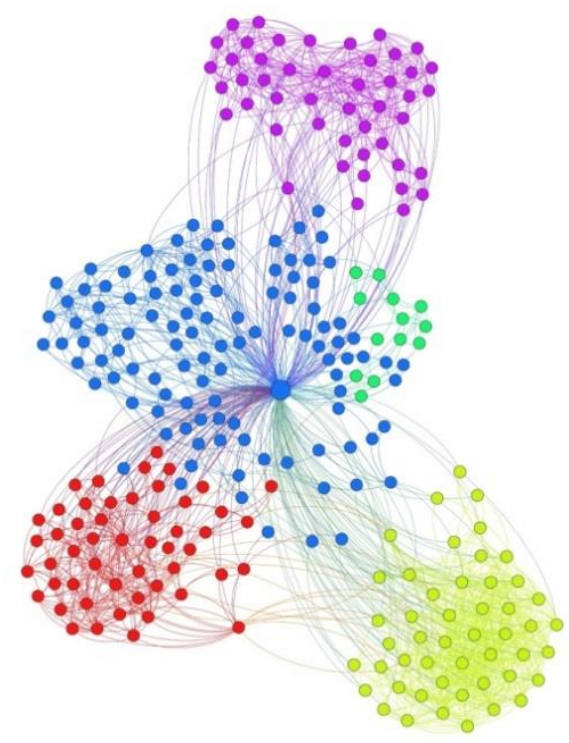

(c)

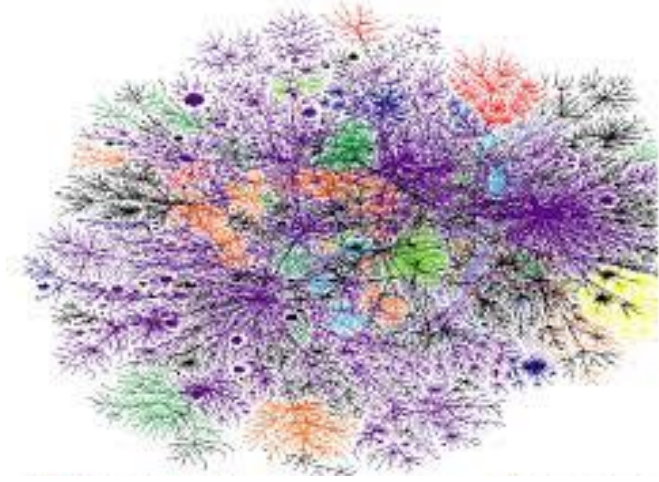

Figure 1.3: Illustrations of networks. (a) A statistical model of the network of connections between brain regions [19]. (b) Friendship network with nodes representing people and links representing friendships. Colors indicate friendship clusters (from Matt Baggott) [18]. (c) Internet network with nodes representing computers and links representing wired or wireless connections between computers [20].

\begin{tabular}{|l|l|l|l|}
\hline Network & Nodes & Links & Node states \\
\hline Internet & Routers & Wires/wireless & Routing state \\
\hline World wide web (www) & Pages & Hyperlinks & Popularity, number of visits \\
\hline Brain & Neurons & Synapses & Electrical (action) potentials \\
\hline Gene regulation & Proteins & Binding sites & Boundness, concentration \\
\hline Ecology web & Species & Competition & Fitness, density \\
\hline Hollywood & Actors & Movies & Celebrity level, contracts \\
\hline
\end{tabular}

Table 1.1: Table showing different systems that can be represented as a network $[25,26]$. 
Networks can be grouped into different categories depending on the connections between nodes. The node connections can be unidirectional, such as food webs and neurons, or bidirectional, such as in computer connectivity.

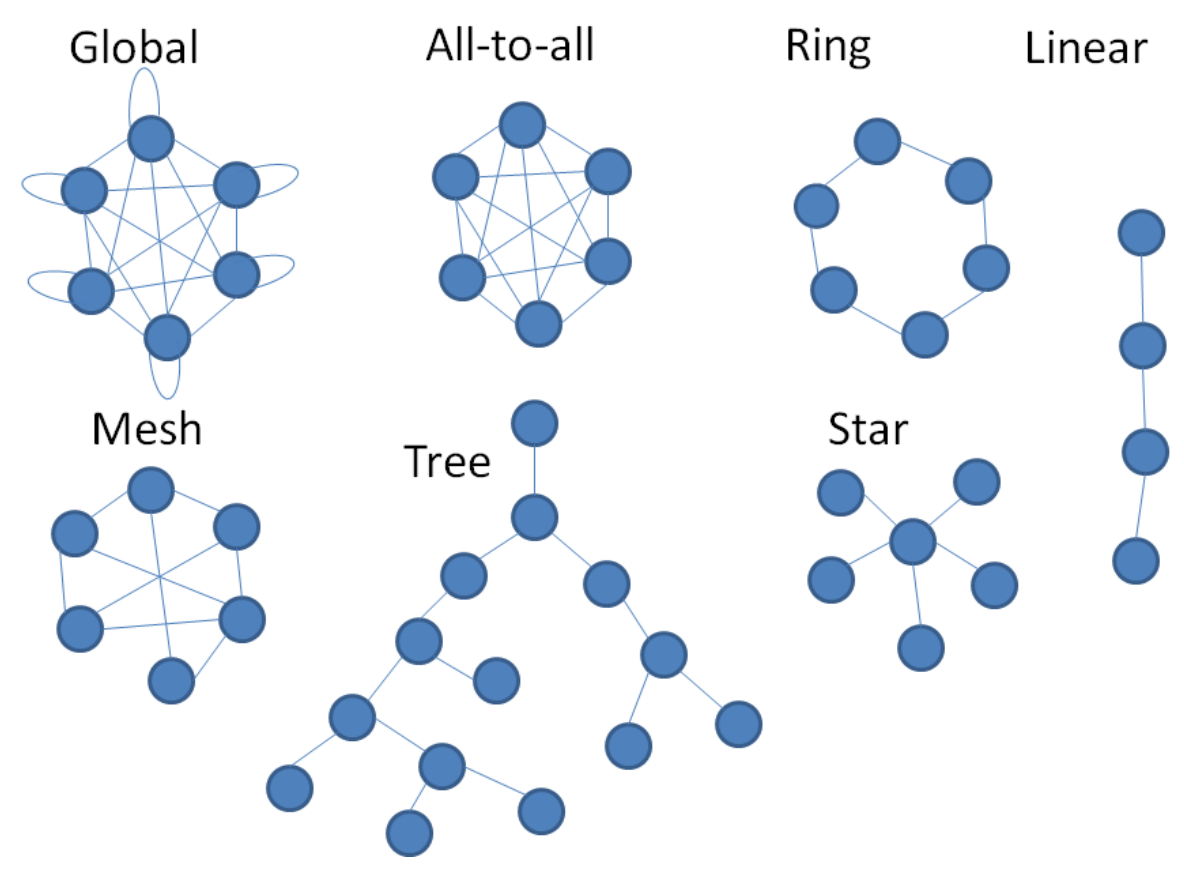

Figure 1.4: Illustration of global, all-to-all, ring, linear, mesh, tree and star network topologies [24].

\subsection{Network in the Kuramoto Model}

To study the effect of a network structure on the collective dynamical behavior of oscillators, we use the Kuramoto model [21,22], which consist of a population of $\mathrm{N}$ coupled phase oscillators, $\theta_{i}(\mathrm{t})$, with a given probability density distribution of the natural frequencies, $\omega_{i}$, given by $g(\omega)$, and the dynamics represented by the phase variable equation below:

$$
\begin{gathered}
\frac{d \theta_{i}}{d t}=\omega_{i}+K \sum_{j=1}^{N} A_{i j} \sin \left(\theta_{i}-\theta_{j}\right), i=1, \ldots \ldots, N, \\
A_{i j}= \begin{cases}1 & j \text { connected to } i \\
0 & j \text { not connected to } i\end{cases}
\end{gathered}
$$

where the adjacency matrix, $A_{i j}$, defines the network and $\mathrm{K}$ is the coupling constant. Each oscillator has its own frequency; however, the coupling tends to synchronize it to all the other oscillators. In synchronization, the interaction of the oscillators gives rise to the individual periods of the oscillators changing to a common period. When the coupling is weak, the 
oscillators are incoherent, whereas above a certain coupling strength, synchronization spontaneously emerges.

The Kuramoto model uses a complex-valued quantity known as the order parameter, $r$, to measure the state of synchronization among the phase oscillators with all-to-all coupling. For a large population of incoherent oscillators, the order parameter increases with increasing coherence:

$$
r e^{i \psi}=\frac{1}{N} \sum_{j=1}^{N} e^{i \theta_{j}}
$$

Here, $0 \leq r(t) \leq 1$ measures the coherence of the oscillator population, and $(t)$ is the average phase. A value of $r(t)$ close to one is for a coherent population of oscillators and a value close to zero is for an incoherent population of oscillators.

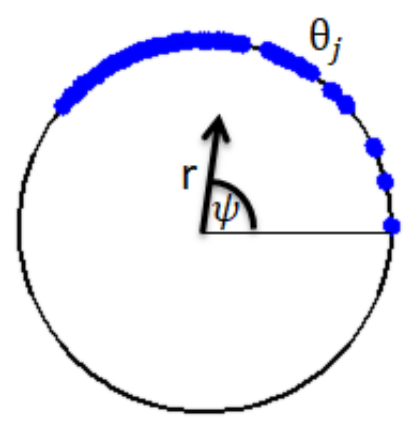

Figure 1.5: Phase plots showing a collection of phase oscillators with an average phase, $\psi$, in the unit circle. The order parameter, $r$, measures the degree of phase synchronization.

In a population of oscillators, the transition from unsynchronized to synchronized occurs above a critical coupling strength. The Kuramoto model defines the critical coupling strength $K_{c}$ as the coupling strength below which the oscillators oscillate with their natural frequencies and the coupling strength above which the oscillators synchronize. A typical order parameter versus coupling strength graph is shown below using BZ oscillators. 


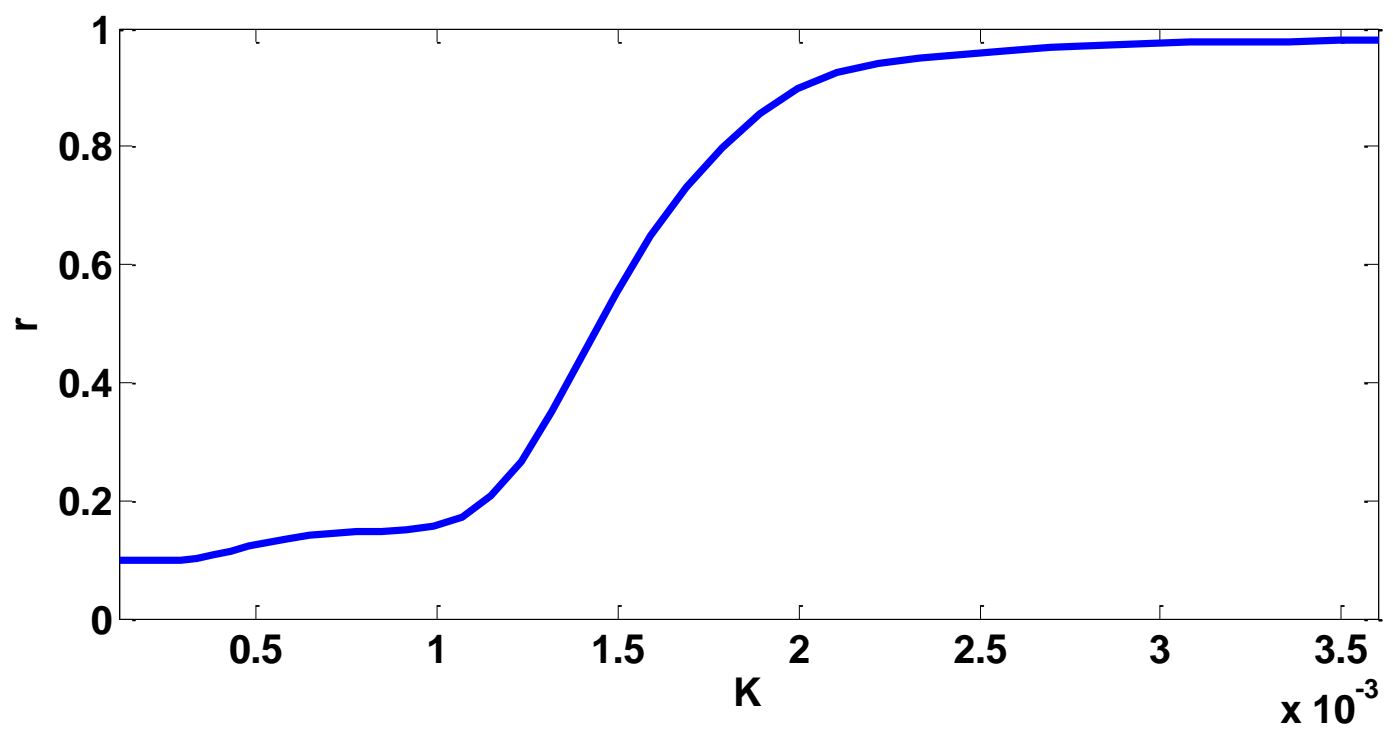

Figure 1.6: Synchronization diagram showing the Kuramoto order parameter, $r$, as a function of $\mathrm{K}$. Typical transition curve from asynchronization to synchronization with the Kuramoto model. The critical coupling strength $K_{c} \approx 1.0 \times 10^{-3}$ for the distribution $g(\omega)$.

For low coupling strength, below $K_{c}$, the oscillators continue to oscillate with their natural frequencies, and they are relatively unaffected by the coupling. When the critical coupling strength is exceeded, there is a quadratic increase of the order parameter with the coupling strength. The synchronization and order parameter described here apply for oscillators with predominately excitatory dynamics.
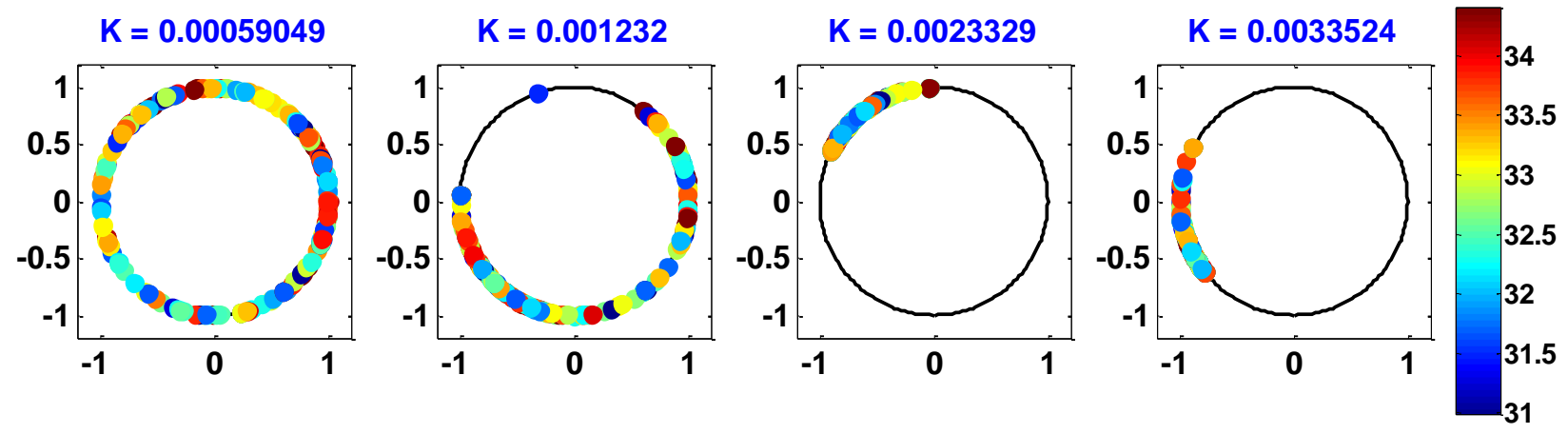

Figure 1.7: Phase plots showing a collection of oscillators in the unit circle, where the color of each oscillator represents its natural period. As coupling strength $\mathrm{K}$ increases, oscillators undergo synchronization. 
Synchronization is a fundamental property of self-organizing systems found throughout nature and technology. A system of oscillators generates a collective rhythm through mutual interactions. Examples of collective rhythms in nature include synchronization of neurons in the brain $[6,23]$, fireflies flashing in unison $[1,2]$, and epidemic outbreaks in ecosystems. In technology, examples of collective rhythms include synchronization of dynamical parts in machines and business cycles within markets. We use the order parameter to measure the state of synchronization of coupled BZ oscillators.

\subsection{Organization of Dissertation}

The dissertation is divided into five Chapters. Chapter 1 introduces synchronization in biological and chemical oscillators, network theory, and in the Kuramoto model. Chapter 2 introduces the BZ reaction mechanism and models. The Belousov-Zhabotinsky reaction was discovered in 1951 by Boris Belousov and was later further developed by Anatol Zhabotinsky. The reaction involves the bromination and oxidation of an organic substrate by bromate in an acidic solution in the presence of a metal ion catalyst. A modification of the BZ reaction mechanism and model to account for the effect of light on the ruthenium catalyzed reaction is described.

Chapter 3 is devoted to describing the experimental discretization of BZ oscillators and the photochemical coupling of BZ oscillators in networks using a spatial light modulator (SLM). Numerical simulation of coupled heterogeneous photosensitive oscillators using the ZBKE model is discussed.

In Chapter 4, we address the phase response curve (PRC) of the BZ reaction for inhibitory-excitatory regimes and synchronization patterns in a pair of coupled photosensitive BZ oscillators. The PRC of an oscillator is useful in determining the entrainment mechanisms of oscillators perturbed by external stimuli. To characterize the PRC of a BZ system, we vary the initial concentration of bromate or bromomalonic acid in the BZ reaction mixture. We report on phase-locked synchronization and autonomous switching patterns in a pair of coupled photosensitive $\mathrm{BZ}$ oscillators. The oscillators, $\mathrm{Ru}(\mathrm{bpy}){ }_{3}^{2+}$-loaded beads in catalyst-free $\mathrm{BZ}$ solutions, were perturbed with light pulses. We quantify the transient changes by constructing the PRC from the resultant oscillations. The PRCs measure the effect of an illumination pulse on the phase of a BZ oscillator. Depending on the phase at which the pulse is applied and the 
chemical composition of the BZ solution, the oscillations are either advanced or delayed relative to the unperturbed oscillations. We present both experimental measurements and numerical simulations of the dynamical behavior of a pair of photochemically coupled BZ oscillators. An analysis of the behavior reveals phase-locked oscillations and periodic and aperiodic switching of oscillations from in-phase to out-of-phase synchronization, which is shown by a phase diagram plot.

Chapter 5 describes how micro-swimmers such as bacteria exhibit various types of collective motion. The dynamical behavior of the individual synthetic micro-swimmers such as platinum-silica particles in hydrogen peroxide solutions has been experimentally studied. However, there are few experimental studies focused on the collective motion of agglomerated micro-swimmers. In chapter 6, we report experiments carried out with silica particles that were asymmetrically coated with Pt and immersed in hydrogen peroxide solutions. We report on the effect of the orientation of the particles in the agglomerate and the resultant motion pattern. Mean square displacement, step length distribution, and mean square angular displacement are used to quantify the resultant motion dynamics. Understanding the influence of orientation of the particles in agglomerates on the motion pattern is important in exploiting this feature of agglomerate micro-swimmers. We report experimental observations of the agglomerate motion and numerical simulations of the agglomerates with different orientations of the particles. We found that motion patterns such as linear or circular trajectories emerge in these agglomerates depending on the particle orientations.

The work is aimed at providing insights into the organizing principles that govern collective behavior in dynamical systems. Using experiments and numerical simulations, we examine the underlying physical principles that enable control and collective behavior in a network of coupled chemical oscillators and self-propelled particles. 


\section{Reference}

[1] J. Buck and E. Buck, "Mechanism of rhythmic synchronous flashing of fireflies. Fireflies of Southeast Asia may use anticipatory time-measuring in synchronizing their flashing," Science 159, 1319-1327 (1968).

[2] J. Buck, E. Buck, J. F. Case, and F E. Hanson, "Control of flashing in fireflies: V. Pacemaker synchronization in Pteroptyxcribellata," J. Comp. Physiol. 144, 287-298 (1981).

[3] Y. Yoshimura and E. M. Callaway, "Fine-scale specificity of cortical networks depends on inhibitory cell type and connectivity," Nat. Neurosci. 8, 1552-1559 (2005).

[4] Y. Yoshimura, J. L. M. Dantzker, and E. M. Callaway, "Excitatory cortical neurons form finescale functional network," Nature 433, 868-873 (2005).

[5] M. Bartos, I. Vida, and P. Jonas, "Synaptic mechanisms of synchronized gamma oscillations in inhibitory interneuron network," Nat. Rev. Neurosci. 8, 45-56 (2007).

[6] E. Marder and D. Bucher, "Central pattern generators and the control of rhythmic movements," Curr. Bio. 11 (13), 986-996 (2001).

[7] E. Bünning, "Circadian rhythms and the time measurement in Photoperiodism," Cold Spring Harb. Symp. Quant. Biol. 25, 249-256 (1960).

[8] S. M. Reppert and D. R. Weaver, "Coordination of circadian timing in mammals," Nature 18, 935-941 (2002).

[9] R. Gao and P. Penzes, "Common mechanisms of excitatory and inhibitory imbalance in schizophrenia and autism spectrum disorders," Curr. Mol. Med. 15 (2), 146-167 (2015).

[10] S. Panda, J. B. Hogenesch, and S. A. Kay, "Circadian rhythms from flies to human," Nature 417, 329-335 (2002).

[11] Y. Cao, H. Wang, Q. Ouyang, and Y. Tu, "The free-energy cost of accurate biochemical oscillations," Nat. Phys. 11, 772 (2015). 
[12] L. Cardelli and A. Csikász-Nagy, "The cell cycle switch computes approximate majority," Nat. Sci. Rep. 2, 656 (2012).

[13] C. E. Robertson, E. Ratai, and N. Kanwisher, "Reduced GABAergic action in the autistic brain," Curr. Bio. 26, 80-85 (2016).

[14] Y. Takumi, L. Bergersen, A. F. Landsend, E. Rinvik, and O. P. Ottersen, "Synaptic arrangement of glutamate receptors," Prog. Brain Res. 116, 105-121 (1998).

[15] P. J. Uhlhaas and W. Singer, "Neural synchrony in brain disorders: Relevance for cognitive dysfunctions and pathophysiology," Neuron 52, 155-168 (2006).

[16] J. H. Barnett, "Early writings on graph theory: Euler circuits and the Königsberg bridge problem," Colorado State University - $\quad$ Pueblo (2005). http://www-users.math.umn.edu/ reiner/Classes/Konigsberg.pdf

[17] R. Shields, "Cultural topology: The seven bridges of Königsberg 1736," Theory Culture and Society 29 (4-5), 43-57 (2012).

[18] https://mattbaggott.wordpress.com/tag/networks/

[19] P. E. Vértes, A. F. Alexander-Bloch, N. Gogtay, J. N. Giedd, J. L. Rapoport, and E. T. Bullmore, “Simple models of human brain functional networks," PNAS 109 (15), 5868-5873 (2012).

[20] Y. Tu, "How robust is the internet?" Nature 406, 6794 (2000).

[21] J. A. Acebrón, L. L. Bonilla, C. J. P. Vicente, F. Ritort, and R. Spigler, "The Kuramoto model: A simple paradigm for synchronization phenomena," Rev. Mod. Phys. 77, 137 (2005).

[22] S. H. Strogatz, "From Kuramoto to Crawford: Exploring the onset of synchronization in populations of coupled oscillators," Phys. D 143, 1-20 (2000).

[23] L. Glass, "Synchronization and rhythmic processes in physiology," Nature 410, 277-284 (2001).

[24] www.kingofnetworking.weebly.com/networktopologies.html 
[25] T. Gross and H. Sayama, Adaptive networks: Theory, models and applications (Understanding complex systems), (Springer Science, 2009).

[26] X. F. Wang and G. Chen, "Complex network: Small-world, scale-free and beyond," IEEE Xplore, 3 (1), 6-20 (2003). 


\section{Chapter Two}

\section{The Belousov-Zhabotinsky (BZ) Reaction}

\subsection{Background/Introduction}

The Belousov-Zhabotinsky (BZ) reaction system is an example of a temporal chemical oscillator - a complex reaction that oscillates with time. A Russian chemist, Boris Belousov, discovered the chemistry that is now known as the Belousov-Zhabotinsky reaction in 1951 [2]. He discovered the reaction accidentally while searching for a Krebs cycle model system. His early attempts to publish his work were rejected because chemists of the time thought that the oscillatory behavior was impossible. It took another decade before Russian biophysicist Anatoli Zhabotinsky, then a graduate student, investigated and further developed the BZ reaction and succeeded in publishing the first results on the system [3]. During the early years following the BZ reaction discovery, the scientific community did not readily accept the BZ reaction as a chemical oscillator. They assumed the reaction would pass its equilibrium point during oscillation, which would violate the second law of thermodynamics. Many scientists compared the $B Z$ reaction to a damped pendulum. In a damped pendulum, during each oscillation, the pendulum passes through its equilibrium position and eventually, comes to rest. However, the BZ reaction involves intermediate reaction steps far from equilibrium [1-5]. The oscillatory behavior in any chemical oscillator is a process involving the intermediates, and the oscillatory behavior occurs far from thermodynamic equilibrium. The BZ reaction is a system of several chemical reactions with dozens of elementary steps. The overall reaction involves the bromination and oxidation of an organic substrate by bromate in an acidic solution in the presence of a metal ion catalyst.

$$
2 \mathrm{BrO}_{3}^{-}+3 \mathrm{CH}_{2}(\mathrm{COOH})_{2}+2 \mathrm{H}^{+} \rightarrow 2 \mathrm{BrCH}(\mathrm{COOH})_{2}+3 \mathrm{CO}_{2}+4 \mathrm{H}_{2} \mathrm{O} \text {. }
$$

The $B Z$ reaction exhibits robust oscillations, and it has become the most widely studied chemical oscillator system. This chapter describes the mechanism and models, such as the

Oregonator and ZBKE models, with their modifications that allow realistic simulations of the dynamic behaviors in the BZ reaction. 


\subsubsection{The BZ Reaction Mechanism}

In 1972, a complete mechanism, known as the FKN mechanism, of the BZ reaction was published by Field, Körös, and Noyes [4]. However, the equations were too complex for numerical analysis. Consequently, Field and Noyes distilled the FKN mechanism to a smaller set of chemical reaction steps [5]. Belousov and Zhabotinsky initially used a cerium-catalyzed BZ reaction system, but Zhabotinsky later showed that other ion complexes, such as ferroin and ruthenium(II) complexes, could also be used in catalyzing the BZ reaction. Autocatalytic oxidation of the metal catalyst by bromous acid, the activator, and the autocatalysis inhibition by the bromide ion, the inhibitor, are important features of the BZ reaction.

The feedback mechanisms of the BZ reaction are illustrated by three processes, which qualitatively describe the mechanism of oscillatory behavior in the BZ system. In the first process, the available bromide is consumed when the bromide reduces the bromate. The first process continues until the bromide concentration drops to a critical level. When the bromide concentration drops below this critical level, the second process begins, involving the bromous acid $\left(\mathrm{HBrO}_{2}\right)$ reduction of bromate, which autocatalytically produces more bromous acid. The bromous acid concentration increases exponentially, which results in the oxidation of the metal catalyst. The autocatalytic process is terminated when the bromous acid converts to hypobromous acid ( $\mathrm{HOBr}$ ) and bromate. Meanwhile, the decomposition of bromomalonic acid and malonic acid in the third process reduces the metal catalyst from the oxidized state and bromide is produced, resetting the initial concentrations so that the process can restart. The different absorption spectra of the oxidation states of the metal catalyst cause the solution to change color as the concentrations change. For the iron catalyst, the oscillations can be clearly observed visually. 
Process 1: The inhibitor bromide ion is consumed to a critical value where the reaction rate of $\mathrm{BrO}_{3}^{-}+\mathrm{HBrO}_{2}$ is the same as reaction rate of $\mathrm{BrO}_{3}^{-}+\mathrm{Br}^{-}$.

$$
\begin{aligned}
& \mathrm{BrO}_{3}^{-}+\mathrm{Br}^{-}+2 \mathrm{H}^{+} \rightarrow \mathrm{HBrO}_{2}+\mathrm{HOBr}, \\
& \mathrm{HBrO}_{2}+\mathrm{Br}^{-}+\mathrm{H}^{+} \rightarrow 2 \mathrm{HOBr} \\
& \mathrm{HOBr}+\mathrm{Br}^{-}+\mathrm{H}^{+} \rightarrow \mathrm{Br}_{2}+\mathrm{H}_{2} \mathrm{O}
\end{aligned}
$$

Process 1 overall: $\mathrm{BrO}_{3}^{-}+5 \mathrm{Br}^{-}+6 \mathrm{H}^{+} \rightarrow 3 \mathrm{Br}_{2}+3 \mathrm{H}_{2} \mathrm{O}$.

Process 2: The Oscillator fires when the system switches to $\mathrm{HBrO}_{2}$ autocatalysis. $\mathrm{HBrO}_{2}$ and $\mathrm{M}^{3+}$ (oxidized metal catalyst) are produced.

$$
\begin{aligned}
& \mathrm{BrO}_{3}^{-}+\mathrm{HBrO}_{2}+\mathrm{H}^{+} \rightarrow 2 \mathrm{BrO}_{2}^{-}+\mathrm{H}_{2} \mathrm{O}, \\
& 2 \mathrm{x}\left(\mathrm{BrO}_{3}^{-}+\mathrm{M}^{2+}+\mathrm{H}^{+} \rightarrow \mathrm{HBrO}_{2}+\mathrm{M}^{3+}\right), \\
& 2 \mathrm{HBrO}_{2} \rightarrow \mathrm{BrO}_{3}^{-}+\mathrm{HOBr}+\mathrm{H}^{+},
\end{aligned}
$$

Process 2 overall: $\mathrm{BrO}_{3}^{-}+\mathrm{HBrO}_{2}+2 \mathrm{M}^{2+}+3 \mathrm{H}^{+} \rightarrow 2 \mathrm{HBrO}_{2}+2 \mathrm{M}^{3+}+\mathrm{H}_{2} \mathbf{O}$.

Process 3: The Oscillator cycle is reset as $\mathrm{M}^{3+}$ regenerates $\mathrm{Br}^{-}$and $\mathrm{M}^{2+}$ (reduced form of metal catalyst).

$$
2 \mathrm{M}^{3+}+\mathrm{MA}+\mathrm{BrMA} \rightarrow 2 \mathrm{M}^{2+}+\mathrm{fBr}^{-}+(\text {prod }) .
$$

In these processes, $\mathrm{BrO}_{3}^{-}$is bromate ion, $\mathrm{Br}^{-}$is bromide ion, $\mathrm{BrMA}$ is brominated malonic acid $\left(\mathrm{BrCH}(\mathrm{COOH})_{2}\right), \mathrm{HBrO}_{2}$ is bromous acid $(\mathrm{O}=\mathrm{Br}-\mathrm{OH}), \mathrm{HOBr}$ is hypobromous acid $(\mathrm{Br}-\mathrm{OH}),[5,9]$.

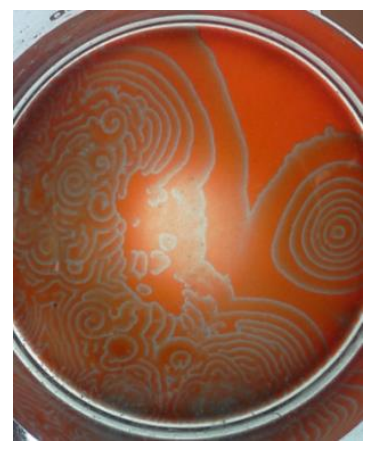

(a)

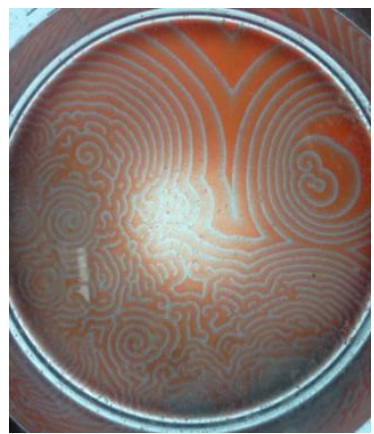

(b)

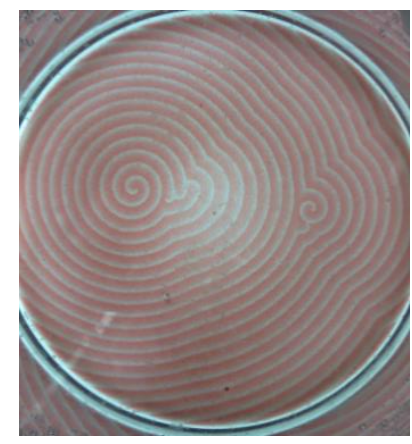

(c)

Figure 2.1: Spatiotemporal pattern formation in the ferroin-catalyzed BZ reaction. The pictures were taken at time intervals of 6 hours from (a) - (c). (Outside the ring the image is reflected by the container walls.) 


\subsubsection{The Oregonator Model}

A mathematical model designed to describe the $B Z$ reaction can be used to simulate the experimental results semi-quantitatively. Field and Noyes reduced the chemical reaction steps from the FKN mechanism to a set of ordinary differential equations with only three variables. They named the model the Oregonator after their home institution, the University of Oregon. The Oregonator model provides a mathematical description of the main features of the reaction, and is given by the equations below [5]:

$$
\begin{aligned}
& \mathrm{A}+\mathrm{Y} \stackrel{\mathrm{k}_{1}}{\rightarrow} \mathrm{X}+\mathrm{P}, \\
& \mathrm{X}+\mathrm{Y} \stackrel{\mathrm{k}_{2}}{\rightarrow} 2 \mathrm{P}, \\
& \mathrm{A}+\mathrm{X} \stackrel{\mathrm{k}_{3}}{\rightarrow} 2 \mathrm{X}+\mathrm{Z}, \\
& 2 \mathrm{X} \stackrel{\mathrm{k}_{4}}{\rightarrow} \mathrm{A}+\mathrm{P}, \\
& \mathrm{B}+\mathrm{Z} \stackrel{\mathrm{k}_{\mathrm{c}}}{\rightarrow} \mathrm{hY},
\end{aligned}
$$

where $\mathrm{A}$ is bromate, $\mathrm{B}$ is all oxidizable organic species, $\mathrm{P}$ is $\mathrm{HOBr}$ (hypobromous acid), $\mathrm{X}$ is $\mathrm{HBrO}_{2}$ (bromous acid), $\mathrm{Y}$ is $\mathrm{Br}^{-}$(bromide), and $\mathrm{Z}$ is the oxidized form of the metal catalyst.

The ordinary differential equations for the three variables $\mathrm{X}, \mathrm{Y}$, and $\mathrm{Z}$ can be written, describing the species $\mathrm{HBrO}_{2}, \mathrm{Br}^{-}$, and the oxidized form of the catalyst, respectively. A and $\mathrm{B}$ correspond to fixed concentrations of the reagents bromate and malonic acid, which are in large excess.

$$
\begin{aligned}
& \frac{d X}{d t}=k_{1} A Y-k_{2} X Y+k_{3} A X-2 k_{4} X^{2} \\
& \frac{d Y}{d t}=-k_{1} A Y-k_{2} X Y+h k_{c} B Z \\
& \frac{d Z}{d t}=2 k_{3} A X-k_{c} B Z
\end{aligned}
$$

The Oregonator model generates limit-cycle oscillations, which are characterized by a constant period. 


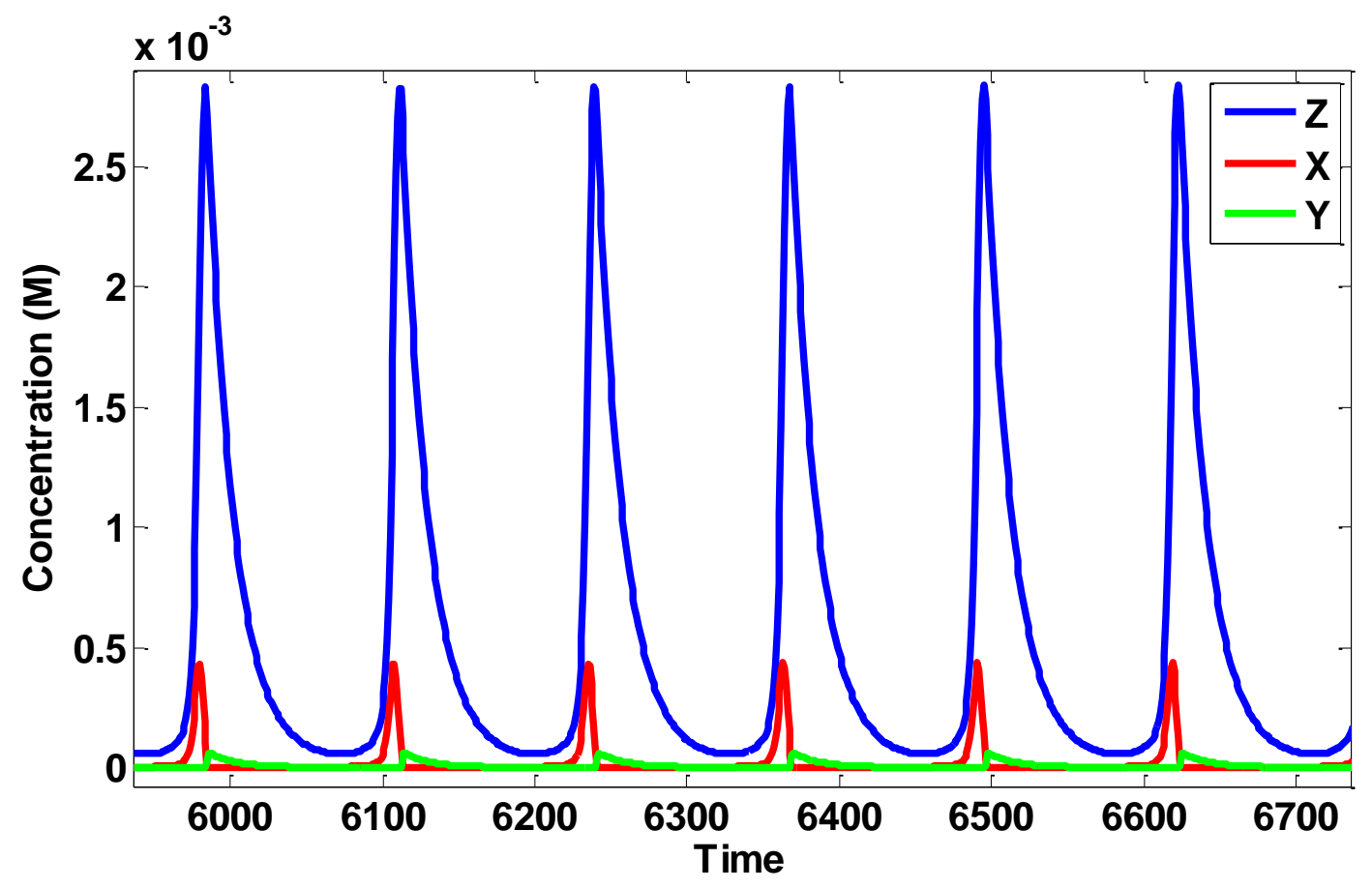

Figure 2.2: Time series showing oscillation for the oxidized catalyst $(\mathrm{Z})$, bromous acid $\left(\mathrm{HBrO}_{2}\right)$ $(X)$, and bromide ion $(Y)$ from Oregonator model simulations of the BZ reaction. The concentrations were calculated by the numerical integration of the differential equations Eq. (2.6) with the rate constants $\mathrm{k}_{1}=6.28, \mathrm{k}_{2}=2.4 \times 10^{6}, \mathrm{k}_{3}=3.0, \mathrm{k}_{4}=240.0, \mathrm{k}_{\mathrm{c}}=1.0$, $h=1.0$, and initial concentrations of bromate $(A)=0.4$ and malonic acid $(B)=0.12$
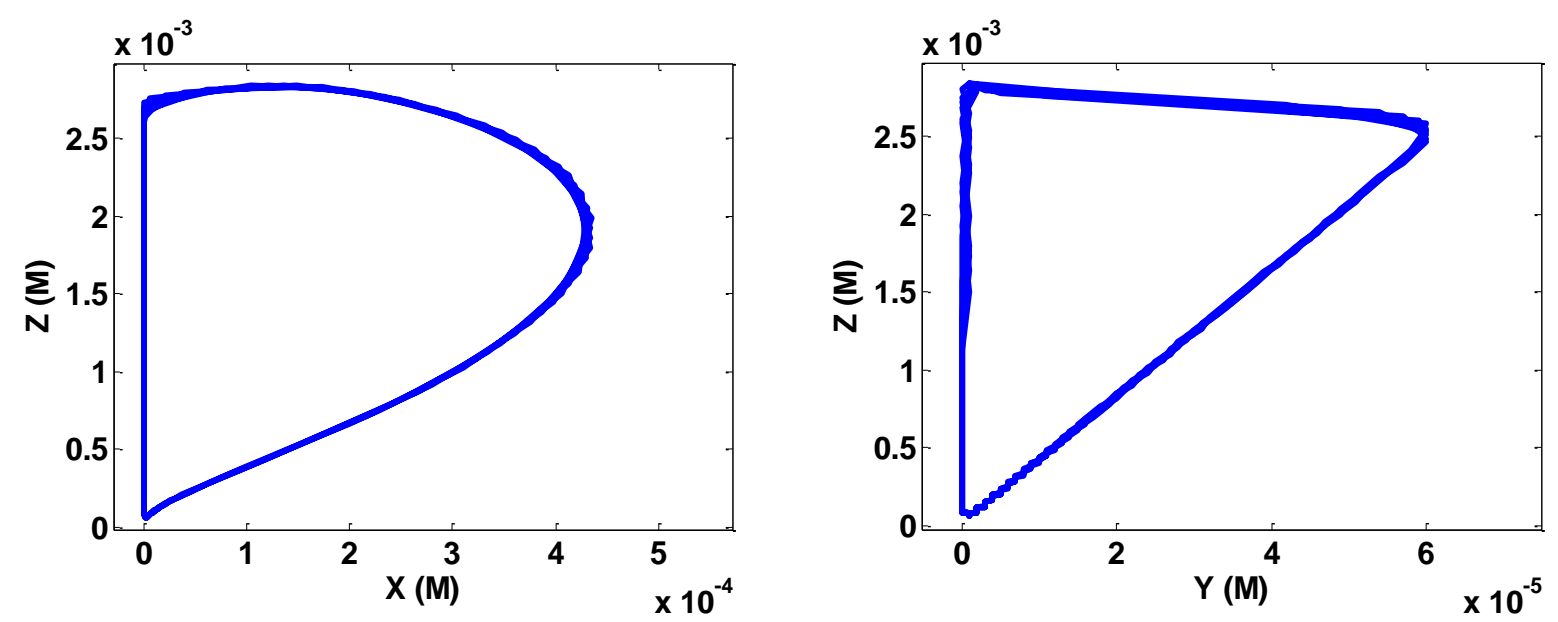

Figure 2.3: Plot of $Z$ vs. $X$ and $Z$ vs. $Y$ showing the limit cycle of the $B Z$ reaction from the Oregonator model simulation. 
The dynamical behavior depends on the concentrations of the reactants and the rate constants. To minimize the number of parameters for analysis, Tyson and Fife $[7,8]$ introduced the non-dimensional variables $\mathrm{u}, \mathrm{w}, \mathrm{v}$, and $\tau$ to replace $\mathrm{X}, \mathrm{Y}, \mathrm{Z}$, and $\mathrm{t}$, respectively, in Eq. (2.6):

$$
\begin{array}{ll}
\mathrm{u}=\left(\frac{2 \mathrm{k}_{4}}{\mathrm{k}_{3} \mathrm{~A}}\right) \mathrm{X}, \quad \mathrm{v}=\left(\frac{\mathrm{k}_{4} \mathrm{k}_{5} \mathrm{~B}}{\left(\mathrm{k}_{3} \mathrm{~A}\right)^{2}}\right) \mathrm{Z}, \quad \mathrm{w}=\left(\frac{\mathrm{k}_{2}}{\mathrm{k}_{3} \mathrm{~A}}\right) \mathrm{Y}, \quad \epsilon=\frac{\mathrm{k}_{5} \mathrm{~B}}{\mathrm{k}_{3} \mathrm{~A}}, \\
\epsilon^{\prime}=\frac{2 \mathrm{k}_{4} \mathrm{k}_{5} \mathrm{~B}}{\mathrm{k}_{2} \mathrm{k}_{3} \mathrm{~A}}, \quad \mathrm{q}=\frac{2 \mathrm{k}_{1} \mathrm{k}_{4}}{\mathrm{k}_{2} \mathrm{k}_{3}}, \quad \tau=\mathrm{k}_{5} \mathrm{Bt}, \quad \mathrm{f}=2 \mathrm{~h},
\end{array}
$$

where $\epsilon, \epsilon^{\prime}$, and $q$ are scaling parameters, and $f$ is the stoichiometry parameter.

The rate equations (2.6) become

$$
\begin{aligned}
& \epsilon \frac{d u}{d \tau}=q w-u w+u-u^{2}, \\
& \frac{d v}{d \tau}=u-v, \\
& \epsilon^{\prime} \frac{d w}{d t}=-q w-u w+f v .
\end{aligned}
$$

Since the values of $\epsilon^{\prime} \ll \epsilon$, the rate of change of $u$ and $v$ will be relatively greater than the rate of change of $\mathrm{w}$. Therefore, the three-variable model can be reduced to a two-variable model by solving for the steady state variable $\mathrm{w}$ :

$$
\mathrm{w}=\frac{\mathrm{fv}}{\mathrm{u}+\mathrm{q}}
$$

Replacing w in Eq. (2.7) above gives

$$
\begin{aligned}
& \frac{\mathrm{du}}{\mathrm{dt}}=\frac{1}{\epsilon}\left[\mathrm{u}-\mathrm{u}^{2}-\mathrm{fv} \frac{\mathrm{u}-\mathrm{q}}{\mathrm{u}+\mathrm{q}}\right] \\
& \frac{\mathrm{dv}}{\mathrm{dt}}=\mathrm{u}-\mathrm{v} .
\end{aligned}
$$

The dimensionless variable $u$ corresponds to $\mathrm{HBrO}_{2}$ and $v$ corresponds to the oxidized state of the catalyst concentrations. The two-variable model retains the qualitative features of the BZ reaction. 


\subsubsection{ZBKE Reaction Mechanism}

To effectively simulate experimental observations, Zhabotinsky, Buchholtz, Kiyatkin, and Epstein proposed a simplified reaction scheme for the bromate-catalyst-bromomalonic acid system, which is known as ZBKE mechanism [1].

\begin{tabular}{|c|c|}
\hline Reaction & Reaction number \\
\hline 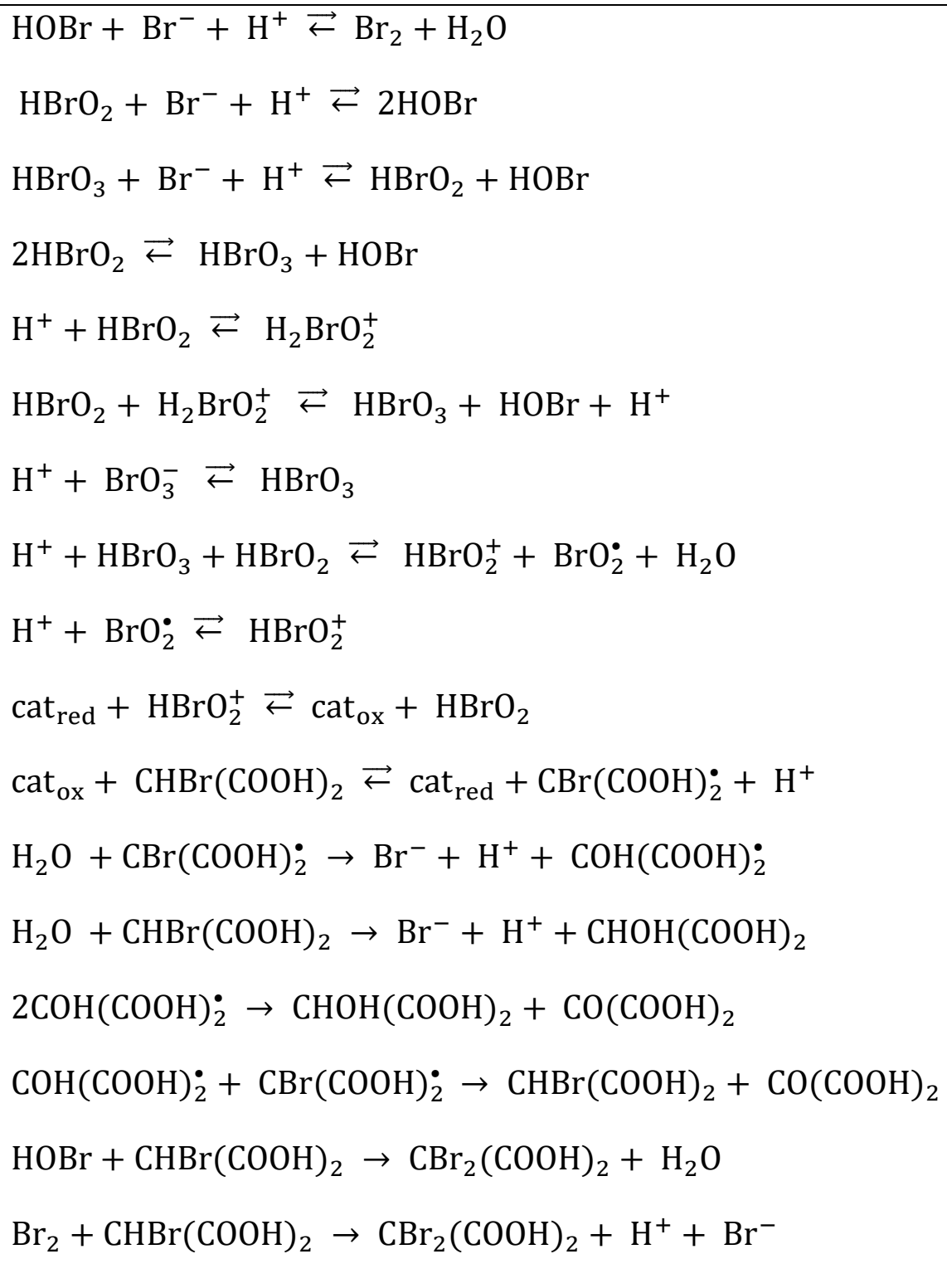 & $\begin{array}{l}\text { ZBKE (R1) } \\
\text { ZBKE (R2) } \\
\text { ZBKE (R3) } \\
\text { ZBKE (R4a) } \\
\text { ZBKE (R4b) } \\
\text { ZBKE (R4C) } \\
\text { ZBKE (R5a) } \\
\text { ZBKE (R5b) } \\
\text { ZBKE (R5C) } \\
\text { ZBKE (R6) } \\
\text { ZBKE (R7) } \\
\text { ZBKE (R8) } \\
\text { ZBKKE (R9) } \\
\text { ZBKE (R10) }\end{array}$ \\
\hline
\end{tabular}

Table 2.1: Table shows the ZBKE mechanism [1], describing the simplified reaction scheme for the bromate-catalyst-bromomalonic acid system. 
The ZBKE mechanism can be reduced to three processes of the BZ reaction system with the essential reaction intermediates for the ZBKE model construction as follows [17]:

Process A: Removal of the inhibitor, $\mathrm{Br}^{-}$.

$$
\begin{aligned}
& \text { (A1) } \mathrm{HBrO}_{3}+\mathrm{Br}^{-}+\mathrm{H}^{+} \rightleftarrows \mathrm{HBrO}_{2}+\mathrm{HOBr} \\
& \text { (A2) } \mathrm{HBrO}_{2}+\mathrm{Br}^{-}+\mathrm{H}^{+} \rightleftarrows 2 \mathrm{HOBr}
\end{aligned}
$$

(A) $\mathrm{HBrO}_{3}+2 \mathrm{Br}^{-}+2 \mathrm{H}^{+} \rightleftarrows 3 \mathrm{HOBr}$

Process B: Autocatalytic production of the activator, $\mathrm{HBrO}_{2}$.

$$
\begin{aligned}
& \text { (B1) } \mathrm{HBrO}_{3}+\mathrm{HBrO}_{2}+2 \mathrm{H}^{+} \rightleftarrows 2 \mathrm{HBrO}_{2}^{+}+\mathrm{H}_{2} \mathrm{O} \\
& \text { (B2) } 2 \mathrm{x}\left(\mathrm{cat}_{\text {red }}+\mathrm{HBrO}_{2}^{+} \rightleftarrows \mathrm{cat}_{\mathrm{ox}}+\mathrm{HBrO}_{2}\right) \\
& \text { (B) } \mathbf{H B r O}_{2}+\mathbf{2 c a t}_{\text {red }}+\mathbf{H B r O}_{3}+\mathbf{2} \mathbf{H}^{+} \rightarrow \mathbf{2} \mathbf{H B r O}_{2}+\mathbf{2} \mathbf{c a t}_{\mathbf{~ o x}}+\mathbf{H}_{2} \mathbf{O}
\end{aligned}
$$

The autocatalytic production of the activator is terminated by step (B3),

$$
\text { (B3) } 2 \mathrm{HBrO}_{2} \rightleftarrows \mathrm{HBrO}_{3}+\mathrm{HOBr}
$$

Process C: Regeneration of the inhibitor, $\mathrm{Br}^{-}$.

$$
\begin{aligned}
& \text { (C1) } \text { cat }_{\mathrm{ox}}+\mathrm{B} \rightleftarrows \text { cat }_{\text {red }}+\mathrm{B}^{\bullet}+\mathrm{H}^{+} \\
& \text {(C2) } \mathrm{B}^{\bullet} \rightarrow \mathrm{fBr}^{-}+\text {products } \\
& \text { (C3) } \mathrm{B} \rightarrow \mathrm{Br}^{-} \\
& \text {(C) } \text { cat }_{\mathrm{ox}}+\mathrm{B} \rightleftarrows \text { cat }_{\text {red }}+\mathbf{f B r}^{-}+\mathbf{H}^{+}+\text {products }
\end{aligned}
$$

\subsubsection{Kinetic Model: ZBKE Model}

The ZBKE model reduces the BZ mechanism to three kinetic variables representing concentrations of three key intermediates. The reaction species involved are defined as $[1,5$, 17]: $\mathrm{X}=\left[\mathrm{HBrO}_{2}\right], \mathrm{Y}=\left[\mathrm{Br}^{-}\right], \mathrm{Z}=\left[\mathrm{cat}_{\mathrm{ox}}\right], \mathrm{U}=\left[\mathrm{HBrO}_{2}^{+}\right], \mathrm{A}=\left[\mathrm{HBrO}_{3}\right]=\mathrm{h}_{0}\left[\mathrm{NaBrO}_{3}\right]_{0} /\left(0.2+\mathrm{h}_{0}\right)$, where $\mathrm{h}_{0}$ is the Hammet acidity function, $\mathrm{H}=\mathrm{h}_{0}, \mathrm{~K}_{\mathrm{HBrO}_{3}}=0.2+\mathrm{h}_{0}, B=\left[\mathrm{CHBr}(\mathrm{COOH})_{2}\right], \mathrm{B}^{\bullet}=$ $\left[\mathrm{CBr}(\mathrm{COOH})_{2}{ }^{\circ}\right], \mathrm{C}=\left(\left[\mathrm{cat}_{\mathrm{ox}}\right]+\left[\mathrm{cat}_{\mathrm{red}}\right]\right), P$ is $\mathrm{HOBr}$, and $\mathrm{Q}$ is organic products. $\mathrm{P}$ and $\mathrm{Q}$ do not affect the dynamics, while $\mathrm{q}$ is a stoichiometric factor. 


$$
\begin{aligned}
& \mathrm{X}+\mathrm{Y}+\mathrm{H} \stackrel{\mathrm{k}_{2}}{\rightarrow} 2 \mathrm{P}, \\
& \mathrm{A}+\mathrm{Y}+\mathrm{H} \stackrel{\mathrm{k}_{3}}{\rightarrow} \mathrm{X}+\mathrm{P}, \\
& 2 \mathrm{X} \stackrel{\mathrm{k}_{4}}{\rightarrow} \mathrm{P}+\mathrm{A}, \\
& \mathrm{A}+\mathrm{X}+\mathrm{H} \underset{\mathrm{k}_{-5}}{\stackrel{\mathrm{k}_{5}}{\rightarrow}} 2 \mathrm{U}, \\
& \mathrm{U}+(\mathrm{C}-\mathrm{Z}) \stackrel{\stackrel{\mathrm{k}_{6}}{\rightarrow}}{\stackrel{\leftarrow}{\mathrm{k}_{-6}}} \mathrm{X}+\mathrm{Z}, \\
& \mathrm{Z}+\mathrm{B} \underset{\mathrm{k}_{-7}}{\stackrel{\mathrm{k}_{7}}{\rightarrow}}(\mathrm{C}-\mathrm{Z})+\mathrm{B}^{\bullet}+\mathrm{H}, \\
& \mathrm{B} \stackrel{\mathrm{k}_{8}}{\rightarrow} \mathrm{qY}+\mathrm{Q}, \\
& \mathrm{B} \stackrel{\mathrm{k}_{9}}{\rightarrow} \mathrm{Y} .
\end{aligned}
$$

$\mathrm{B}$ is bromomalonic acid, $\mathrm{P}$ is hypobromous acid, $\mathrm{X}$ is bromous acid, $\mathrm{Y}$ is bromide, and $\mathrm{Z}$ is the oxidized form of the metal catalyst.

The ordinary differential equations for the three variables $\mathrm{X}, \mathrm{Y}$, and $\mathrm{Z}$ describing the species $\mathrm{HBrO}_{2}, \mathrm{Br}^{-}$, and the oxidized form of the catalyst, respectively, make up the mathematical model. $A, h_{0}$, and $B$ are held constant, as the concentrations of bromate, sulfuric acid, and malonic acid in the $B Z$ reaction are in large excess.

$$
\begin{aligned}
\frac{d X}{d T}= & -k_{2} h_{o} X Y+k_{3} h_{o} A Y-2 k_{4} X^{2}-k_{5} h_{o} A X+k_{-5} U_{s s}^{2}+ \\
& k_{6} U_{s s}(C-Z)-k_{-6} X Z \\
\frac{d Y}{d T}= & -k_{2} h_{0} X Y-k_{3} h_{0} A X+q\left[\frac{k_{7} k_{8} B Z}{k_{8}+k_{-7} h_{o}(C-Z)}\right]+k_{9} B \\
\frac{d z}{d T}= & k_{6} U_{s s}(C-Z)-k_{-6} X Z-\left[\frac{k_{7} k_{8} B Z}{k_{8}+k_{-7} h_{o}(C-Z)}\right]
\end{aligned}
$$

The parameter $U_{s s}$ is the concentration of $U$ at steady state and can be calculated as follows:

$$
\mathrm{U}_{\mathrm{ss}}=\frac{1}{4 \mathrm{k}_{-5}}\left[\mathrm{k}_{6}(\mathrm{c}-\mathrm{z})+\sqrt{\mathrm{k}_{6}^{2}(\mathrm{c}-\mathrm{z})^{2}+16 \mathrm{k}_{5} \mathrm{k}_{-5} \mathrm{~h}_{\mathrm{o}} \mathrm{Ax}+8 \mathrm{k}_{-5} \mathrm{k}_{-6} \mathrm{xz}}\right]
$$


The following differential equations describe the non-dimensional ZBKE oscillator's dynamics [20]:

$$
\begin{aligned}
& \frac{\mathrm{dx}}{\mathrm{dt}}=\frac{1}{\varepsilon_{1}}\left(-\mathrm{x}(\mathrm{x}+\mathrm{y}+1)+\varepsilon_{2} \gamma_{1} \mathrm{p}^{2}+\mathrm{p}(1-\mathrm{z})+\mu \mathrm{y}\right) \\
& \frac{\mathrm{dy}}{\mathrm{dt}}=\frac{1}{\varepsilon_{4}}\left(-\mathrm{y}(\mathrm{x}+\mu)+\mathrm{q} \alpha \frac{\mathrm{z}}{\varepsilon_{3}+1-\mathrm{z}}+\beta\right) \\
& \frac{\mathrm{dz}}{\mathrm{dt}}=\mathrm{p}(1-\mathrm{z})-\alpha \frac{\mathrm{z}}{\varepsilon_{3}+1-\mathrm{z}}
\end{aligned}
$$

The dimensionless state variables $\mathrm{x}, \mathrm{y}$, and $\mathrm{z}$ are the concentrations of $\mathrm{HBrO}_{2}, \mathrm{Br}^{-}$, and the oxidized form of the catalyst respectively. The non-dimensional steady state concentration of $\mathrm{U}_{\mathrm{ss}}$ is designated $p$ and can be calculated as follows:

$$
\begin{aligned}
& \mathrm{p}=-\frac{\gamma_{1}}{4 \varepsilon_{2}}\left(1-\mathrm{z}-\sqrt{(\mathrm{z}-1)^{2}+16 \gamma_{1} \varepsilon_{2} \mathrm{x}}\right) \\
& \mathrm{x}=\frac{\mathrm{k}_{5} \mathrm{~h}_{0}}{2 \mathrm{k}_{4}} \mathrm{AX}, \quad \mathrm{y}=\frac{\mathrm{k}_{5}}{\mathrm{k}_{2}} \mathrm{AY}, \quad \mathrm{z}=\mathrm{CZ}, \\
& \mathrm{u}=\frac{\left(\mathrm{k}_{5} \mathrm{~h}_{0} \mathrm{~A}\right)^{2} \mathrm{U}}{2 \mathrm{k}_{4} \mathrm{k}_{6} \mathrm{C}}, \quad \mathrm{t}=\frac{2 \mathrm{k}_{4} \mathrm{CZ}}{\left(\mathrm{k}_{5} \mathrm{~h}_{0} \mathrm{~A}\right)^{2}}
\end{aligned}
$$

These constant parameters contain information about the rate equations of the irreversible steps of the reduced mechanism and the concentration of the malonic acid.

$$
\begin{array}{lll}
\varepsilon_{1}=\frac{\mathrm{k}_{5} \mathrm{~h}_{0} \mathrm{~A}}{2 \mathrm{k}_{4} \mathrm{C}}, & \varepsilon_{2}=\frac{\left(\mathrm{k}_{5} \mathrm{~h}_{0} \mathrm{~A}\right)^{2}}{2 \mathrm{k}_{4} \mathrm{k}_{6} \mathrm{C}}, & \varepsilon_{3}=\frac{\mathrm{k}_{8}}{\mathrm{k}_{-7} \mathrm{~h}_{0} \mathrm{C}} \\
\varepsilon_{4}=\frac{\mathrm{k}_{5} \mathrm{~A}}{\mathrm{k}_{2} \mathrm{C}^{\prime}} & \alpha=\frac{2 \mathrm{k}_{4} \mathrm{k}_{7} \mathrm{k}_{8} \mathrm{~B}}{\mathrm{k}_{5}^{2} \mathrm{k}_{-7} \mathrm{~h}_{0}^{3} \mathrm{~A}^{2}}, & \beta=\frac{2 \mathrm{k}_{4} \mathrm{k}_{9} \mathrm{~B}}{\left(\mathrm{k}_{5} \mathrm{~h}_{0} \mathrm{~A}\right)^{2}} \\
\mu=\frac{2 \mathrm{k}_{3} \mathrm{k}_{4}}{\mathrm{k}_{2} \mathrm{k}_{5} \mathrm{~h}_{0}}, & \gamma=\frac{\mathrm{k}_{-5}}{\mathrm{k}_{6}}, & \delta=\frac{\mathrm{k}_{-6} \mathrm{C}}{\mathrm{k}_{5} \mathrm{~h}_{0} \mathrm{~A}}
\end{array}
$$




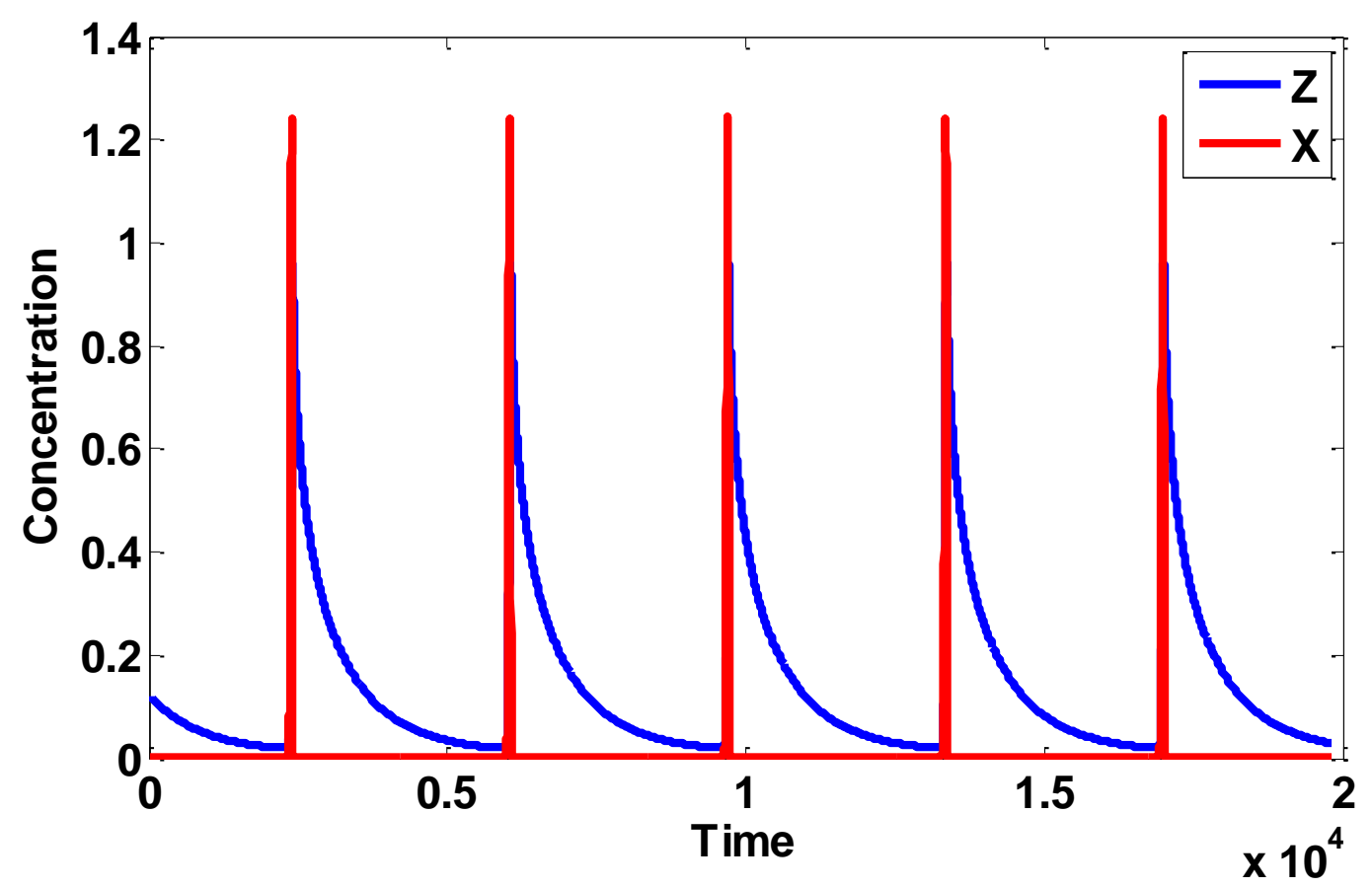

Figure 2.4: Time series showing the oscillations of the oxidized form of the catalyst $(Z)$ and bromous acid $(X)$ from ZBKE model simulations of the $B Z$ reaction system.
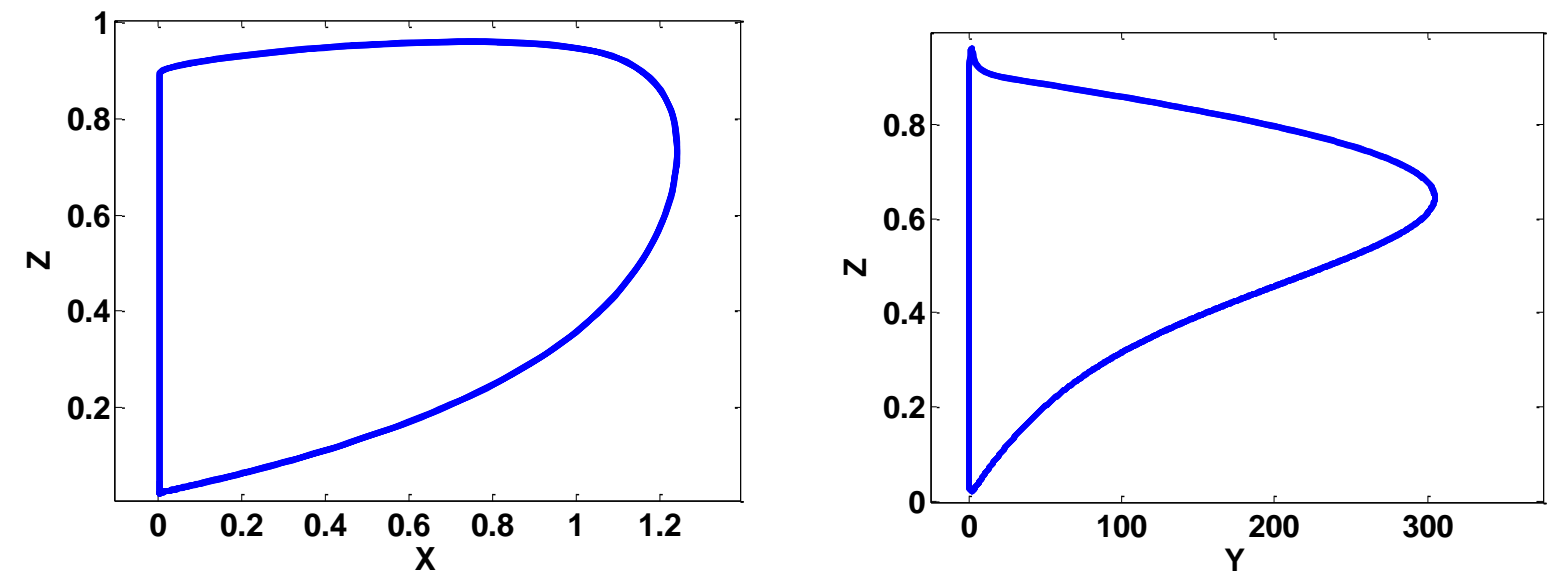

Figure 2.5: A plot of $Z$ vs. $X$ and $Z$ vs. $Y$ showing the limit cycle of the $B Z$ reaction from ZBKE model simulations.

The parameter values used for the ZBKE simulations follow:

$$
\begin{aligned}
& \varepsilon_{1}=0.11, \quad \varepsilon_{2}=1.7 \times 10^{-5}, \quad \varepsilon_{3}=1.6 \times 10^{-3}, \quad \varepsilon_{4}=4.2 \times 10^{-4}, \\
& \alpha=0.1, \quad \beta=1.7 \times 10^{-5}, \quad \mu=2.4 \times 10^{-4}, \quad \gamma=1.2, \quad q=0.35-1.18
\end{aligned}
$$




\subsubsection{Effect of Light on the BZ Reaction}

The photosensitivity of the catalyzed BZ reaction to illumination has been a subject of a number of studies. Researchers have carried out studies on photoinhibition and photoinduction by ultraviolet (UV) light irradiation of a cerium-catalyzed BZ reaction[12, 13]. In 1973, Demas and Diemente [14] used the $\mathrm{Ru}(\mathrm{bpy})_{3}{ }^{2+}$ catalyst as a luminescent indicator in the $\mathrm{BZ}$ reaction system. Using a batch reactor, Gáspár et al. [8] investigated the illumination effects on the $\mathrm{Ru}(\mathrm{bpy})_{3}{ }^{2+}$-catalyzed $\mathrm{BZ}$ reaction in 1983. In the study, ruthenium catalyst was photochemically excited by $440-460 \mathrm{~nm}$ light. The period of the oscillation in the $\mathrm{Ru}(\mathrm{bpy})_{3}{ }^{2+}$-catalyzed $\mathrm{BZ}$ reaction was altered by the light. Photoinduction and photoinhibition processes were observed in subsequent studies of the $B Z$ reaction $[8,9,18]$. The sensitivity to an illumination pulse in the $\mathrm{Ru}(\mathrm{bpy})_{3}{ }^{2+}$-catalyzed $\mathrm{BZ}$ reaction was found to be altered when the initial concentration of bromomalonic acid was varied. The response of the BZ reaction is attributed to two channels of photochemical reaction [9]. These processes involve the photochemical production of the bromide ion from $\mathrm{BrMA}$ and the photochemical production of $\mathrm{HBrO}_{2}$ from the bromate ion.

\subsubsection{The Photo-Chemical Mechanism}

Through the two reaction channels, the light sensitivity of both the inorganic and organic subsets of the Ru-catalyzed BZ reaction is incorporated in the overall reaction mechanism as shown below $[9,18]$.

$$
\mathrm{Ru}(\mathrm{II})+\mathrm{h} v \rightarrow \mathrm{Ru}(\mathrm{II})^{*}
$$

\section{Excitatory Channel}

$$
\begin{aligned}
& \mathrm{Ru}(\mathrm{II})^{*}+\mathrm{BrO}_{3}^{-}+2 \mathrm{H}^{+} \rightarrow \mathrm{BrO}_{2}^{-}+\mathrm{Ru}(\mathrm{III})+\mathrm{H}_{2} \mathrm{O} \\
& \mathrm{BrO}_{2}^{+}+\mathrm{Ru}(\mathrm{II})+\mathrm{H}^{+} \rightarrow \mathrm{HBrO}_{2}+\mathrm{Ru}(\mathrm{III})
\end{aligned}
$$

Inhibitory Channel

$$
\begin{aligned}
& \mathrm{Ru}(\mathrm{II})^{*}+\mathrm{BrMA} \rightarrow \mathrm{Ru}(\mathrm{III})+\mathrm{Br}^{-}+\text {org. prod. } \\
& \mathrm{Ru}(\mathrm{III})+\mathrm{BrMA} \rightarrow \mathrm{Ru}(\mathrm{II})+\mathrm{Br}^{-}+\text {org. prod. }
\end{aligned}
$$

Bromous acid is photochemically produced from bromate through the excitatory channel while bromide is photochemically generated from BrMA through the inhibitory channel. The 
bromomalonic acid that forms during the induction period is a bromide source that is critical in determining whether the oscillator will be photoinhibitory or photoexcitatory. Thus, the ratio of bromomalonic acid to bromate affects the BZ reaction and the photosensitive properties of the Ru-catalyst. On light perturbation, the two reaction processes in Eq. (2.17) and Eq. (2.18) compete. When the ratio of bromomalonic acid to bromate is below a critical value, then photoexcitation is seen with the application of light. If the ratio is above the critical value, then photoinhibition results from a light perturbation.

\subsubsection{Light sensitive ZBKE model modification}

In 1990, Krug et al. [15], proposed a model for the photosensitivity in the BZ reaction. The modification included in the Oregonator model accounted for the excitatory effects of visible light illumination. In 1997, Kádár et al. [9], further modified the model to account for the photoinhibitory effects of visible light irradiation. The inhibiting effects are due to the photochemical generation of bromide ions from bromomalonic acid, while excitatory effects are a result of bromous acid photochemically produced from bromate. The equations accounting for the photosensitivity of the BZ reaction system in the ZBKE model are shown

below. The reaction species are defined as $\mathrm{E}=\mathrm{Ru}(\mathrm{bpy}){ }_{3}^{2+*}$ (excited catalyst), $\mathrm{A}=$ bromate, $\mathrm{B}=$ bromomalonic acid (BrMA), $\mathrm{X}=$ bromous acid (activator), $\mathrm{Y}=$ bromide ion (inhibitor), and $\mathrm{Z}=$ $\mathrm{Ru}(\mathrm{bpy})_{3}{ }^{3+}$ (oxidized catalyst) [9].

\section{Photo-excitatory channel}

$$
\mathrm{E}+\mathrm{A} \rightarrow \mathrm{X}+2 \mathrm{Z}
$$

Photoinhibitory channel

$$
\mathrm{E}+\mathrm{B} \rightarrow \mathrm{Y}+\mathrm{Z}
$$

We use the ZBKE model to carry out numerical simulations to compare with the experimental BZ reaction measurements. The influence of these two photochemical processes in the overall sensitivity to a light pulse can be included in the ZBKE oscillator dynamics by modification of the differential equations using a parameter $\phi[16]$. 


$$
\begin{aligned}
& \frac{\mathrm{dx}}{\mathrm{dt}}=\frac{1}{\varepsilon_{1}}\left(\phi-\mathrm{x}(\mathrm{x}+\mathrm{y}+1)+\varepsilon_{2} \gamma_{1} \mathrm{p}^{2}+\mathrm{p}(1-\mathrm{z})+\mu \mathrm{y}\right), \\
& \frac{\mathrm{dy}}{\mathrm{dt}}=\frac{1}{\varepsilon_{4}}\left(\phi-\mathrm{y}(\mathrm{x}+\mu)+\mathrm{q} \alpha \frac{\mathrm{z}}{\varepsilon_{3}+1-\mathrm{z}}+\beta\right), \\
& \frac{\mathrm{dz}}{\mathrm{dt}}=\phi+\mathrm{p}(1-\mathrm{z})-\alpha \frac{\mathrm{z}}{\varepsilon_{3}+1-\mathrm{z}} .
\end{aligned}
$$

Depending on whether the BZ reaction system is excitatory or inhibitory, parameter $\phi$ appears in the Eq. (2.21) or Eq. (2.22), respectively. In an excitatory system, $\phi$ does not appear in Eq. (2.22), while in an inhibitory system, $\phi$ does not appear in Eq. (2.21).

The above modification is limited to either pure excitatory or pure inhibitory behavior, which does not capture all of the dynamics of the experimental observations. We modify the model further by introducing a tunable parameter, $\alpha_{0}$, that accounts for the photochemical processes that take place when light is applied to the Ru-catalyzed BZ reaction. The model below includes photochemical terms $\alpha_{0}$ and $\phi$ in the ZBKE model for the photosensitive BZ system.

$$
\begin{aligned}
& \frac{\mathrm{dx}}{\mathrm{dt}}=\frac{1}{\varepsilon_{1}}\left(\alpha_{0} \phi-\mathrm{x}(\mathrm{x}+\mathrm{y}+1)+\varepsilon_{2} \gamma_{1} \mathrm{p}^{2}+\mathrm{p}(1-\mathrm{z})+\mu \mathrm{y}\right) \\
& \frac{\mathrm{dy}}{\mathrm{dt}}=\frac{1}{\varepsilon_{4}}\left(\left(1-\alpha_{0}\right) \phi-\mathrm{y}(\mathrm{x}+\mu)+\mathrm{q} \alpha \frac{\mathrm{z}}{\varepsilon_{3}+1-\mathrm{z}}+\beta\right), \\
& \frac{\mathrm{dz}}{\mathrm{dt}}=\left(1+\alpha_{0}\right) \phi+\mathrm{p}(1-\mathrm{z})-\alpha \frac{\mathrm{z}}{\varepsilon_{3}+1-\mathrm{z}}
\end{aligned}
$$

The parameter $\alpha_{0}$ is introduced to specify the weight of the excitatory and inhibitory channels in the Ru-catalyzed BZ reaction when a light pulse of intensity $\phi$ is applied. The system is purely excitatory for $\alpha_{0}=1.0$ and purely inhibitory for $\alpha_{0}=0.0$. 


\section{References}

[1] A. M. Zhabotinsky, F. Buchholtz, A. B. Kiyatkin, and I. R. Epstein, "Oscillations and waves in metalion-catalyzed bromate oscillating reactions in highly oxidized states." J. Phys. Chem. 97, 7578 (1993).

[2] A. Pechenkin, “BP Belousov and his reaction." J. Biosci, 34(3), 365-371, 2009.

[3] A. M. Zhabotinsky, "A history of chemical oscillations and waves." Chaos 1, 379 - 386 (1991).

[4] R. J. Field, E. Körös, and R. M. Noyes, "Oscillations in chemical systems. II. Thorough analysis of temporal oscillation in the bromate-cerium-malonic acid system." J. Am. Chem. Soc. 94, 8649-8664 (1972).

[5] R. J. Field and R. M. Noyes, "Oscillations in chemical systems. IV. Limit cycle behavior in a model of a real chemical reaction." J. Chem. Phys. 60, 1877-1884 (1974).

[6] J. J. Tyson, "Scaling and reducing the Field-Körös-Noyes mechanism of the Belousov-Zhabotinsky reaction." J. Chem. Phys 86, 3006-3012 (1982).

[7] J. J. Tyson and P. Fife, "Target patterns in a realistic model of the Belousov-Zhabotinsky reaction." J. Chem. Phys. 3, 2224-2237 (1980).

[8] V. Gáspár, G. Bazas, and M. T. Beck, "The influence of visible light on the Belousov-Zhabotinskii oscillating reactions applying different catalysts." Z. Phys. Chem. (Leipzig) 264, 43-48 (1983).

[9] S. Kádár, T. Amemiya, and K. Showalter, "Reaction mechanism for light sensitivity of the Ru(bpy) ${ }_{3}^{2+}$ catalyzed Belousov-Zhabotinsky reaction." J. Phys. Chem. A 101, 8200-8206 (1997).

[10] I. R. Epstein and K. Showalter, "Nonlinear chemical dynamics: Oscillations, patterns, and chaos." J. Phys. Chem. 100, 13132-13147 (1996).

[11] A. S. Mikhailov and K. Showalter, "Control of waves, patterns and turbulence in chemical systems." Phys. Rep. 425, 79-194 (2006).

[12] V. A. Vavilin, A. M. Zhabotinsky, and A. N. Zaikin, "Effect of ultraviolet radiation on the self-oscillator oxidation of malonic acid derivatives." Russ. J. Phys. Chem. 42, 091-094 (1963).

[13] H. Buse and B. Hess, "Information transmission in a diffusion-coupled oscillatory chemical system." Nature 244, 203-205 (1973).

[14] J. N. Deas and D. J. Diemente, "An oscillating chemical reaction with a luminescent indicator." J. Chem. Ed. 50, 357-358 (1973).

[15] H. J. Krug, L. Pohlmann, and L. Kuhnert, "Analysis of the modified complete Oregonator accounting for oxygen sensitivity and photosensitivity of Belousov-Zhabotinsky systems." J. Phys. Chem. 94, 48624866 (1990). 
[16] V. K. Vanag, L. Yang, M. Dolnik, A. Zhabotinsky, and I. R. Epstein, "Oscillatory cluster patterns in a homogeneous chemical system with global feedback." Nature 406, 389-391 (2000).

[17] H. Sevcikova, I. Schreiber, and M. Marek, "Dynamics of oxidation Belousov-Zhabotinsky waves in an electric field." J. Phys. Chem. 100, 9153-19164 (1996).

[18] I. Hanazaki, Y. Mori, T. Sekiguchi, and G. Rabai, "Photo-response of chemical oscillators." Phys. D 84, 228-237 (1995).

[19] A. F. Taylor, P. Kapetanopoulos, B. J. Whitaker, R. Toth, L. Bull, and M. R. Tinsley, "Phase clustering in globally coupled photochemical oscillators." Eur. Phys. J. Spec. Top. 165, 137-149 (2008).

[20] R.Toth, A. F. Taylor, and M. R. Tinsley, "Collective behavior of a population of chemically coupled oscillators."J. Phys. Chem. B 110, 10170 (2006).

[21] J. Maselko and K. Showalter, "Chemical Waves on Spherical Surfaces" Nature 339, 609-611 (1989).

[22] J. Maselko, J. S. Reckley, and K. Showalter, "Regular and Irregular Spatial Patterns in an Immobilized-Catalyst Belousov-Zhabotinsky Reaction." J. Phys. Chem. 93, 2774-2780.

[23] A. F. Taylor, M. R. Tinsley, F. Wang, Z. Huang, K. Showalter, "Phase Clusters in Large Populations of Chemical Oscillators." Science 323, 614 (2009).

[24] M. R. Tinsley, A. F. Taylor, Z. Huang, K. Showalter, "Emergence of Collective Behavior in Groups of Excitable Catalyst-Loaded Particles: Spatiotemporal Dynamical Quorum Sensing." Phys. Rev. Lett. 102, 158301 (2009).

[25] M. R. Tinsley, S. Nkomo, and K. Showalter, "Chimera and phase-cluter states in populations of coupled chemical oscillators." Nat. Phys 8, 662-665 (2012).

[26] S. Nkomo, M. R. Tinsley, and K. Showalter, "Chimera states in populations of nonlocally coupled chemical oscillators." Phys. Rev. Lett. 110, 24402 (2013). 


\section{Chapter Three}

\section{Discretization and Coupling}

\subsection{Discretization of the BZ Reaction System}

Belousov's original experiment used a well-stirred solution to study temporal oscillations [1]. However, subsequent studies using an unstirred catalyzed BZ reaction in solutions [2, 3], gels [4], and membranes $[5,6,7,8]$ revealed the formation of spatial patterns. In the unstirred system, the intermediate species propagate by diffusion and initiate reaction in adjacent regions.

A BZ system with cation exchange beads loaded with the catalyst in a catalyst-free reaction solution was first studied by Maselko and Showalter [10, 11]. Depending on the size of cation exchange beads and the reaction mixture, uniform pattern formation, irregular behaviors, and broken waves were observed. Maselko and Showalter [9] also studied the spatiotemporal behavior of the BZ reaction on the bead surface. Uniform oxidation and reduction of the catalyst, propagation of waves, and formation of spirals were observed on the surface of these beads.

The use of cation exchange beads provides an effective means of discretizing the BZ reaction oscillation. Each discrete bead can be thought of as a single BZ reaction oscillatory unit. Discretizing the reaction system enables the study of collective behaviors in a population of the BZ oscillators. Collectively, these chemical oscillators can interact by mutual signaling in the form of stimuli that alters the behavior of the oscillators. Coupling a population of discrete BZ oscillators has allowed researchers to study emergent behaviors such as synchronization [12, $15,18]$, desynchronization [19], quorum sensing [14], and chimera states [16, 17].

In order to study populations of coupled BZ oscillators, coupling methods must be developed. Using the BZ reaction system with catalyst-loaded beads, two coupling methods have been utilized: diffusive coupling and photochemical coupling. Diffusive coupling involves the exchange of chemical species with the surrounding solution. On the other hand, photochemical coupling involves the light-induced production of chemical species by the oscillatory unit due to excitation of the photosensitive metal catalyst. In diffusive coupling, 
behaviors such as synchronization and quorum sensing have been studied, while photochemical coupling has allowed the study of synchronization and chimera states in a networks of BZ oscillators. Synchronization occurs when oscillators fire either simultaneously or with a constant phase difference, while quorum sensing is an oscillator density dependent behavior [15]. The chimera state is where the population of oscillators splits into coherent and incoherent groups. The photochemical coupling allows interactions of BZ oscillators to take place by timed light pulses, which enables the study of BZ oscillators in a network.

\subsection{Photochemical Coupling of BZ Oscillators in a Network}

The photosensitivity property has been successfully utilized in the coupling of discrete $\mathrm{Ru}(\mathrm{bpy})_{3}{ }^{2+}$-catalyzed BZ reaction oscillators [16-19]. Experiments have been carried out that involve coupling a population of $\mathrm{Ru}(\mathrm{bpy})_{3}{ }^{2+}$-catalyzed BZ oscillators in a network [18]. Using $\mathrm{Ru}(\mathrm{bpy})_{3}{ }^{2+}$-loaded beads in catalyst-free BZ solutions, arbitrary network topologies can be realized by photochemical coupling. This system has been used to investigate synchronization, chimera states, phase-lag synchronization, echo behavior, and desynchronization phenomena [16 - 20].

Discretizing the illumination pulse applied on the oscillator allows us to model a spatiotemporal network using an adjacency matrix for coupling the oscillators. Networks consist of nodes and links. In our case, the nodes are the oscillators and the links are the connections between the oscillators. Consider individual oscillators labeled $i=1,2, \ldots, N$. The interaction process of these oscillators is determined by an $N$ by $N$ matrix, $\mathrm{A}_{\mathrm{ij}}$. Thus, a network topology is established by introducing adjacency matrix, $A_{i j}$, to the coupling equation. The individual oscillators occupy the nodes of the network. The links determine the interaction of the oscillators. If $A_{i j}=1$, there is a link from node $i$ to $j$. If $A_{i j}=0$, there is no link leading from node $i$ to $j$. The example matrix $\mathrm{A}_{\mathrm{ij}}$ below has undirected links.

$$
A_{i j}=\left\{\begin{array}{c}
1 \text { if } j \text { is connected to } i \\
0 \text { if } j \text { is not connected to } i
\end{array}\right.
$$




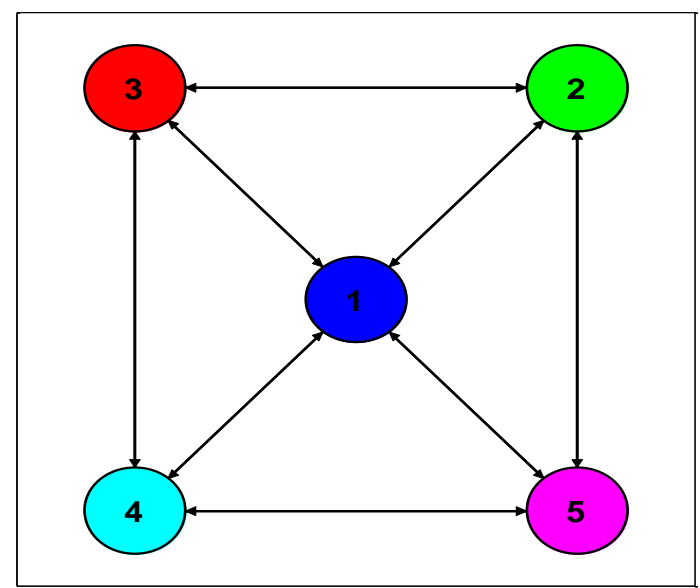

Figure 3.1: Network of five coupled oscillators.

$$
\mathrm{A}_{\mathrm{ij}}=\begin{array}{rrrrr}
0 & 1 & 1 & 1 & 1 \\
1 & 0 & 1 & 0 & 1 \\
1 & 1 & 0 & 1 & 0 \\
1 & 0 & 1 & 0 & 1 \\
1 & 1 & 0 & 1 & 0
\end{array}
$$

Adjacency matrix for the network in Figure 3.1.

The photochemical coupling between two oscillators $i$ and $j$ is carried out by determining the difference between the gray level of oscillator $i, z_{i}$, and gray level of oscillator $j, z_{j}$, and multiplying this difference by the coupling constant, $\delta$. The illumination pulse, $\varphi_{\mathrm{i}}$, is applied to the respective discrete oscillators via a space- and time-dependent feedback. The light intensity feedback, $\varphi_{i}$ is calculate by:

$$
\varphi_{\mathrm{i}}=\delta \sum_{\mathrm{j}=1}^{\mathrm{n}} \mathrm{A}_{\mathrm{ij}}\left(\mathrm{z}_{\mathrm{i}}-\mathrm{z}_{\mathrm{j}}\right)
$$

The coupling constant is $\delta$ and $\mathrm{A}_{\mathrm{ij}}$ is the adjacency matrix, which introduces the network topology. 


\subsection{Experimental Methods}

\subsubsection{BZ Reaction Mixture}

The experiments were carried out using a photosensitive BZ reaction system in which catalytic $\mathrm{Ru}(\mathrm{bpy}){ }_{3}{ }^{2+}$-loaded cation exchange beads were dispersed in catalyst-free $\mathrm{BZ}$ solutions. Each discrete bead represents a single oscillatory unit. The catalyst-free BZ solutions used for the experiments were prepared from commercially available analytical grade chemicals with deionized water. The catalyst-free BZ reaction mixture consists of the following reagents: sodium bromide $(\mathrm{NaBr})$, sodium bromate $\left(\mathrm{NaBrO}_{3}\right)$, sulfuric acid $\left(\mathrm{H}_{2} \mathrm{SO}_{4}\right)$, and malonic acid $\left(\mathrm{CH}_{2}(\mathrm{COOH})_{2}\right)$.

\subsubsection{Ruthenium (II) Loaded Oscillator Bead Preparation}

The $\mathrm{Ru}(\mathrm{bpy})_{3}{ }^{2+}$-loaded beads were prepared by mixing $1.0 \mathrm{ml}$ of $0.025 \mathrm{M} \mathrm{Ru(bpy)}{ }_{3}{ }^{2+}$ with 3.0 grams cation exchange beads (DOWEX 50WZ2-100; Sigma-Aldrich) and $9.0 \mathrm{ml}$ of deionized water. The mixture is stirred for 12-24 hours. The beads are filtered and air-dried (under dim light) before use. The dry beads are stored in a glass vial, under dim light conditions. The beads are inherently heterogeneous since individual beads have small differences in size and different adsorbed catalyst concentrations. The inherent heterogeneity causes the oscillators to have a distribution of natural oscillation periods.

\subsubsection{The Oscillator System Preparation}

The catalyst-free BZ solution is prepared by mixing reagents with initial concentrations, depending on the reaction regime listed in Table 3.1, to obtain the desired reaction concentrations. The discrete catalyst-loaded beads were dispersed and arranged in a glass petri dish filled with catalyst-free BZ solution of height approximately 2.0-3.0 mm. The beads are separated by at least two diameters to avoid diffusive coupling, as shown in Figure 3.2. 


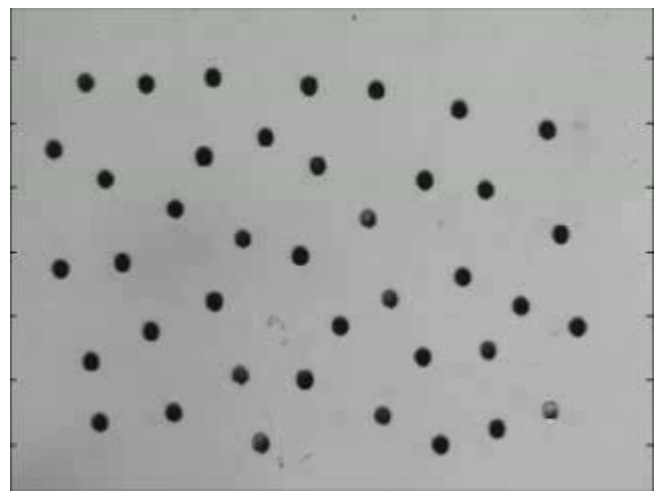

Figure 3.2: Catalytic $\mathrm{Ru}(\mathrm{bpy})_{3}{ }^{2+}$-loaded cation exchange beads (200-250 microns in diameter) in catalyst-free Belousov-Zabotinsky (BZ) solutions.

\begin{tabular}{|c|c|c|c|c|}
\hline \multirow[b]{2}{*}{ BZ Reagents } & \multicolumn{4}{|c|}{ Initial Concentrations (M) for Different Reaction Regimes } \\
\hline & $\begin{array}{l}\text { Excitatory } \\
\text { (E) }\end{array}$ & $\begin{array}{l}\text { Bromate varied } \\
(I->E)\end{array}$ & $\begin{array}{l}\text { Bromide varied } \\
\text { (E }>\text { I) }\end{array}$ & $\begin{array}{l}\text { Inhibitory } \\
\text { (I) }\end{array}$ \\
\hline MA & 0.08 & 0.096 & 0.096 & 0.08 \\
\hline $\mathrm{H}_{2} \mathrm{SO}_{4}$ & 0.78 & 0.72 & 0.72 & 0.78 \\
\hline $\mathrm{NaBr}$ & 0.02 & 0.06 & $0.02-0.09$ & 0.09 \\
\hline $\mathrm{NaBrO}_{3}$ & 0.48 & $0.32-0.76$ & 0.48 & 0.48 \\
\hline $\mathrm{Ru}(\text { bipy })_{3}{ }^{2+}$-catalyst & $8.3 \times 10^{-6} \mathrm{~mol} / \mathrm{g}$ & $8.3 \times 10^{-6} \mathrm{~mol} / \mathrm{g}$ & $8.3 \times 10^{-6} \mathrm{~mol} / \mathrm{g}$ & $8.3 \times 10^{-6} \mathrm{~mol} / \mathrm{g}$ \\
\hline
\end{tabular}

Table 3.1: Initial concentrations for the catalyst-free BZ solution and catalyst-loaded oscillator beads.

\subsubsection{Instrumental Setup}

The experimental setup is shown by the schematic diagram in Figure 3.3. The setup includes a glass petri dish (reactor), a beam splitter, a bandpass filter, and a spatial light modulator (SLM) connected to and controlled by a computer coupled to a charge-coupled device (CCD)-camera. The SLM projects the light, and the beam splitter reflects the light onto the reaction vessel while allowing real time observation with the camera. A bandpass filter improves the contrast of the grayscale signal from the photosensitive tris(2,2'bipyridine)ruthenium(II) loaded beads at $440-460 \mathrm{~nm}$. The projected light also serves as a 
background for observing the chemical oscillators. During an oscillation cycle, the beads change color based on the concentrations of the reduced, $\mathrm{Ru}(\mathrm{bipy})_{3}{ }^{2+}$ (orange), and oxidized, $\mathrm{Ru}\left(\right.$ bipy) ${ }_{3}{ }^{3+}$ (green), forms of the BZ catalyst. The color changes on the discrete beads can be processed in grayscale values from the images taken with the CCD-camera. The grayscale values of individual beads are plotted in real time.

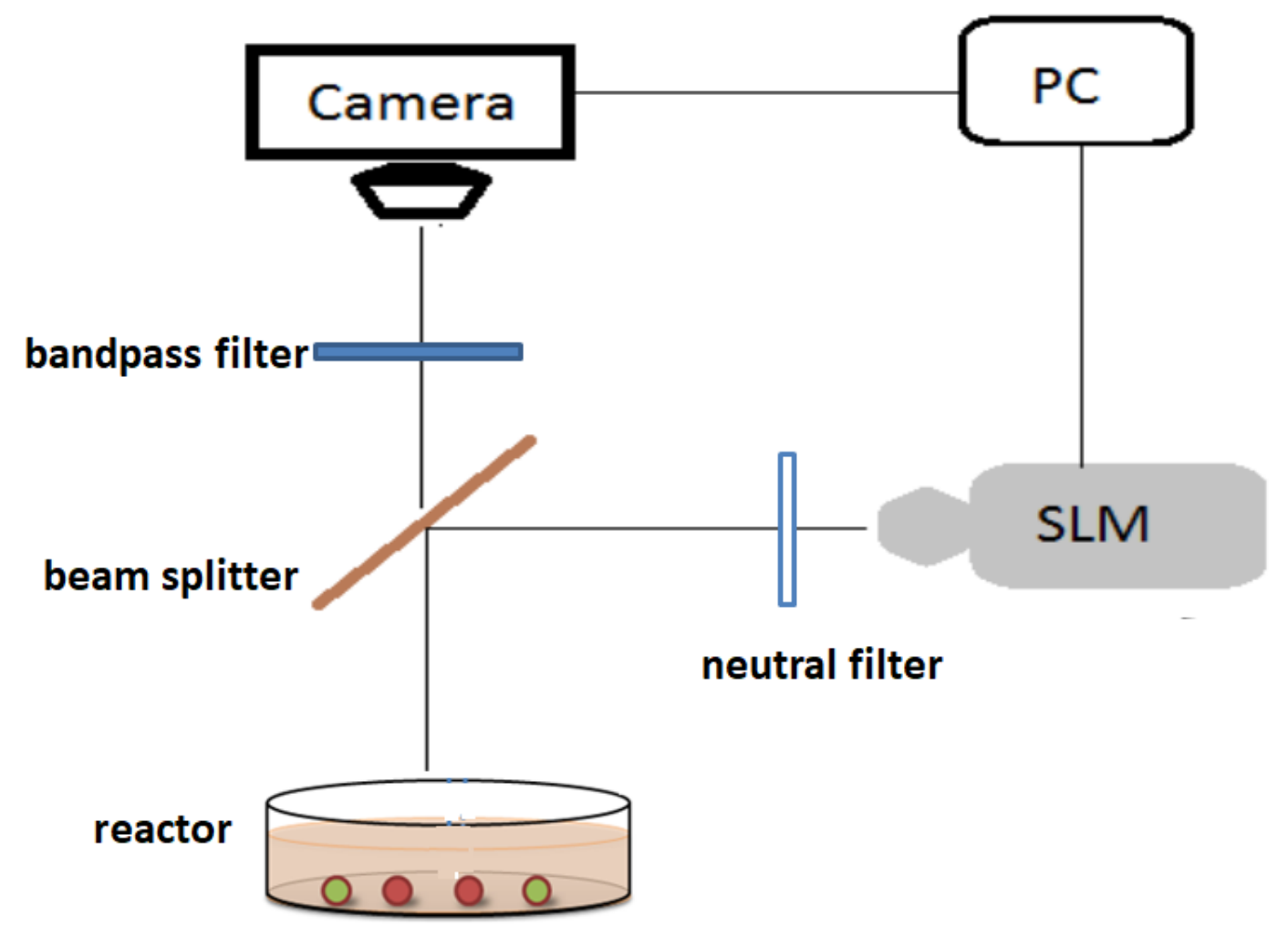

Figure 3.3: Schematic diagram of the experimental setup for the BZ reaction system with discrete Ru-catalyst loaded beads [18].

\subsubsection{Computer-Control and Photochemical Coupling}

The experiment is carried out with a duty cycle of 2.8 seconds, controlled by MatLab algorithms. A single cycle consists of exposing the reaction vessel to background light while the CCD-camera captures an image, followed by the light perturbation according to the feedback and the cycle then repeats. The CCD-camera sends images of the spatiotemporal behavior of the individual beads as shown in Figure 3.2 to the computer every cycle. The computer records the intensity (averaged grayscale value) of each bead from the image, and the measured 
intensity of individual beads is plotted. When coupling is turned on, the measured averaged grayscale values of the discrete beads can be used to calculate the illumination feedback according to Eq. (3.3). The feedback is applied for a duration of 1.5 seconds to the beads via irradiation with the SLM. Then the background light is turned on, another image is captured, and the cycle repeats.

The photochemical coupling between two beads $i$ and $j$ is carried out by determining the difference in the transmitted light intensity of the beads, in grayscale, and multiplying this difference by the normalized coupling constant, $\delta=255 \frac{\mathrm{k}}{\mathrm{d}_{\mathrm{i}}}$, according to Eq. (3.3):

$$
\varphi_{\mathrm{i}}=\varphi_{0}+255 \frac{\mathrm{k}}{\mathrm{d}_{\mathrm{i}}} \sum_{\mathrm{j}=1}^{\mathrm{n}} \mathrm{A}_{\mathrm{ij}}\left(\mathrm{z}_{\mathrm{i}}-\mathrm{z}_{\mathrm{j}}\right) \text {. }
$$

The light with intensity $\varphi_{i}$ is projected by the SLM onto a defined area where the individual bead $i$ is positioned. The normalized grayscale values $z_{i}$ and $z_{j}$ are proportional to the $\mathrm{Ru}$ (bipy) ${ }_{3}{ }^{3+}$ concentration of beads $i$ and $j$, respectively. The normalized coupling constant, $\delta$, the projected light intensities, $\varphi_{i}$, and background light intensity, $\varphi_{0}$, in Eq. (3.3) are dimensionless. The light intensity of grayscale value 100 corresponds to $0.925 \mathrm{~mW} \mathrm{~cm}^{-2}$ and the actual value of the applied light intensity can be calculated by multiplying the grayscale value of $\varphi_{i}$ by $9.25 \times 10^{-3} \mathrm{~mW} \mathrm{~cm}^{-2}$. The experimental time for determining the gray levels of beads $i$ and $j$ and projecting the light with intensity $\varphi_{i}$ on bead $i$ is approximately 0.4 seconds.

For light-induced phase resetting experiments, the SLM illuminates with a grayscale value of 250 , corresponding to $\mathrm{I}=2.31 \mathrm{~mW} \mathrm{~cm}^{-2}$, to individual beads in defined time frames (a sequence of three pulses separated by 1.3 seconds; for our experiment a total of 4.5 seconds illumination time was used). The background light is then turned on, an image is captured, and the cycle repeats. When there is no specific illumination, the background light intensity is resumed. 


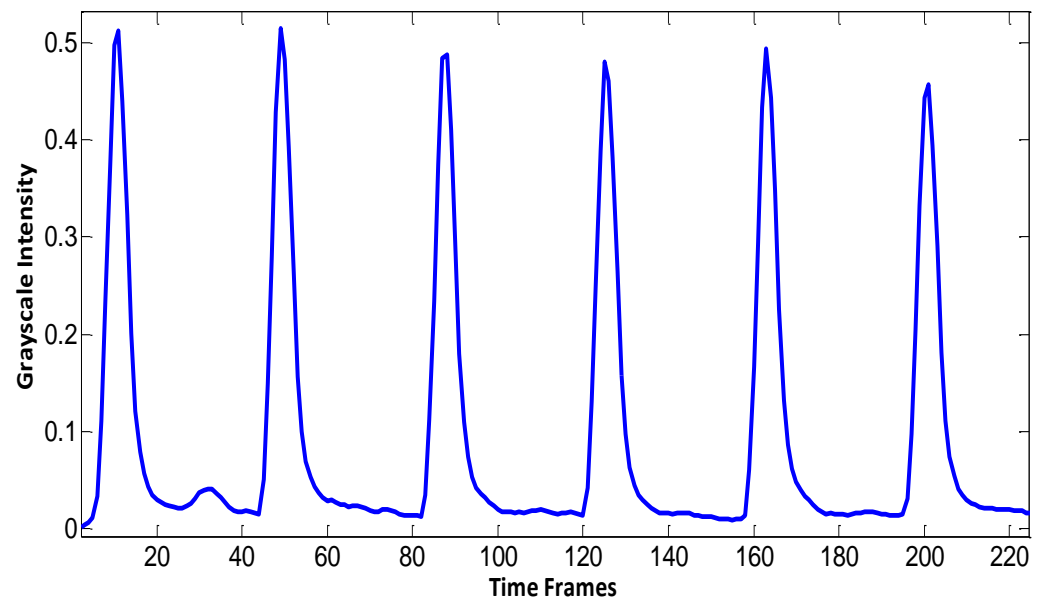

Figure 3.4: Time series showing grayscale values of Ru-catalyst loaded bead.

\subsection{Numerical Simulations: Coupled ZBKE Oscillator Model}

The chemical reaction taking place on each catalyst-loaded bead and the influence of the projected light perturbations on the reaction are numerically simulated with a modified ZBKE model for the photosensitive BZ reaction. For oscillators interacting via coupling, Eq. (2.22) is modified by replacing the parameter $\varphi$ by the coupling function $\varphi_{i}$, which characterizes the interaction between the coupled oscillators. Following the experimental setup for the coupled chemical oscillators, the model has been extended to include a term representing a feedback $\varphi_{i}$ on $N$ oscillators, label $i=1,2, \ldots, N$, whose individual dynamics are given by Eq. (3.4) below:

$$
\begin{aligned}
& \frac{\mathrm{dx}_{\mathrm{i}}}{\mathrm{dt}}=\frac{1}{\varepsilon_{1}}\left(\alpha_{0} \varphi_{\mathrm{i}}-\mathrm{x}_{\mathrm{i}}\left(\mathrm{x}_{\mathrm{i}}+\mathrm{y}_{\mathrm{i}}+1\right)+\varepsilon_{2} \gamma_{1} \mathrm{p}_{\mathrm{i}}^{2}+\mathrm{p}_{\mathrm{i}}\left(1-\mathrm{z}_{\mathrm{i}}\right)+\mu \mathrm{y}_{\mathrm{i}}\right), \\
& \frac{\mathrm{dy_{ \textrm {i } }}}{\mathrm{dt}}=\frac{1}{\varepsilon_{4}}\left(\left(1-\alpha_{0}\right) \varphi_{\mathrm{i}}-\mathrm{y}_{\mathrm{i}}\left(\mathrm{y}_{\mathrm{i}}+\mu\right)+\mathrm{q}_{\mathrm{i}} \alpha \frac{\mathrm{z}_{\mathrm{i}}}{\varepsilon_{3}+1-\mathrm{z}_{\mathrm{i}}}+\beta\right), \\
& \frac{\mathrm{dz} \mathrm{z}_{\mathrm{i}}}{\mathrm{dt}}=\left(1+\alpha_{0}\right) \varphi_{\mathrm{i}}+\mathrm{p}_{\mathrm{i}}\left(1-\mathrm{z}_{\mathrm{i}}\right)-\alpha \frac{\mathrm{z}_{\mathrm{i}}}{\varepsilon_{3}+1-\mathrm{z}_{\mathrm{i}}} .
\end{aligned}
$$

The constant values used in the ZBKE model simulations are listed below:

$$
\begin{array}{ll}
\varepsilon_{1}=0.11, & \alpha=0.10, \\
\varepsilon_{2}=1.7 \times 10^{-5}, & \beta=1.7 \times 10^{-5}, \\
\varepsilon_{3}=1.6 \times 10^{-2}, & \mu=2.4 \times 10^{-4},
\end{array}
$$




$$
\varepsilon_{4}=4.2 \times 10^{-4}, \quad \gamma_{1}=1.10,
$$

where $\varphi_{i}$ is given by

$$
\varphi_{\mathrm{i}}=\mathrm{K} \sum_{\mathrm{j}=1}^{\mathrm{n}} \mathrm{A}_{\mathrm{ij}}\left(\mathrm{z}_{\mathrm{i}}-\mathrm{z}_{\mathrm{j}}\right)
$$

where $K, \mathrm{~A}_{\mathrm{ij}}$, and $z$ are the coupling constant, adjacency matrix, and oxidized form of the catalyst respectively.

When studying a population of oscillators, the initial numerical simulation conditions can be set to homogeneous oscillators by using a single value of $q_{i}$, with $0.35<q_{i}<1.180$, where oscillations are observed. However, the interacting dynamical units in many real systems are not homogeneous, as pointed out earlier with the case of heterogeneous Ru-loaded beads in the BZ reaction. Some examples in nature include cells in the hippocampus and cortex, which are known to be diverse in terms of their biochemical content, morphology, electrophysiological characteristics, and neuromodulator sensitivities with theta and gamma having frequency bands of $(8-12 \mathrm{~Hz})$ and $(20-80 \mathrm{~Hz})$, respectively [13]. Networks of these cells have been found to be responsible for the generation and control of rhythmic brain activities. Studying the influence of intrinsic properties such as heterogeneity provides insights into the generation and control of collective behaviors in populations of coupled oscillators. Therefore, we investigated the effects of heterogeneities on the local dynamics of the BZ system using experimental methods and numerical simulations. We introduce heterogeneity in the modified ZBKE model by assigning the parameter values $q_{i}$ with the distribution $q_{i} \in[0.35,1.18]$, where these values are within the oscillatory regime. 


\section{Reference}

[1] A. Pechenkin, "BP Belousov and his reaction." J. Biosci, 34(3), 365-371, 2009.

[2] R. J. Field and R. M. Noyes, "Oscillations in chemical systems. V. Quantitative explanation of band migration in the Belousov-Zhabotinskii reaction." J. Am. Chem. Soc. 96(7), 2001-2006 (1974).

[3] J. Ross, S. C. Müller, and C. Vidal, “Chemical waves.” Science. 240 (4851), 460-465 (1988).

[4] B. Neumann, Z. Nagyungvarai, and S. C Müller, "Interaction between silica gel matrices and the Belousov-Zhabotinsky reaction." Chem. Phys. Lett. 95(15), 5831-5837 (1991).

[5] O. Steinbock, P. Kettunen, and K. Showalter, "Anisotropy and spiral organizing centers in patterned excitable media." Sciene, 269 (5232), 1857-1860 (1995).

[6] A. Lazar, Z. Noszticzius, HD. Forsterling, and Z. Nagy-Ungvarai, "Chemical waves in modified membranes I. Developing the technique." Physica D, 84 (1-2), 112-119 (1995).

[7] A. Lazar, Z. Noszticzius, H. Farkas, and HD. Forsterling, "Involutes: the geometry of chemical waves rotating in annular membranes." Chaos. 5(2), 443-447 (1995).

[8] D. Winston, M. Arora, J. Maselko, V.Gaspar, and K. Showalter, "Cross-membrane coupling of chemical spatiotemporal patterns." Nature, 351 (6322), 132-135 (1991).

[9] J. Maselko and K. Showalter, "Chemical waves on spherical surfaces." Nature 339, 609-611 (1989).

[10] J. Maselko, J. S. Reckley, and K. Showalter, "Regular and irregular spatial patterns in an immobilized-catalyst Belousov-Zhabotinsky reaction." J. Phys. Chem., 93 (7), 2774-2780 (1989).

[11] J. Maselko, and K. Showalter, "Chemical waves in inhomogeneous excitable media." Physica D, 9 (1-2), 21-32 (1991).

[12] R. Toth, A. F. Taylor, and M. R. Tinsley, "Collective behavior of a population of chemically coupled oscillators."J. Phys. Chem. B 110, 10170 (2006).

[13] A. Bragin, G. Jando, Z. Nadasdy, J. Hetke, K. Wise, and G. Buzsaki, "Gamma 40-100 Hz oscillation in the hippocampus of the behaving rate." J. Neurosci 15, 47-60 (1995).

[14] A. F. Taylor, M. R. Tinsley, F. Wang, Z. Huang, K. Showalter, "Phase clusters in large populations of chemical oscillators." Science 323, 614 (2009). 
[15] M. R. Tinsley, A. F. Taylor, Z. Huang, K. Showalter, "Emergence of collective behavior in groups of excitable catalyst-loaded particles: Spatiotemporal dynamical quorum sensing." Phys. Rev. Lett. 102, 158301 (2009).

[16] M. R. Tinsley, S. Nkomo, and K. Showalter, "Chimera and phase-cluster states in populations of coupled chemical oscillators." Nat. Phys 8, 662-665 (2012).

[17] S. Nkomo, M. R. Tinsley, and K. Showalter, "Chimera states in populations of nonlocally coupled chemical oscillators." Phys. Rev. Lett. 110, 24402 (2013).

[18] J. Totz, R. Snari, D. Yengi, M. R. Tinsley, H. Engel, K. Showalter, "Phase-lag synchronization in networks of coupled chemical oscillators.", Phys. Rev. E 92, 022819 (2015)

[19] R. Snari, M. R. Tinsley, D. Wilson, S. Faramrzi, T. I. Netoff, J. Moehlis, and K. Showalter, "Desynchronization of stochastically synchronized chemical oscillators." Chaos 25, 123116 (2015)

[20] T. Chen, M. R. Tinsley, E. Ott, and K. Showalter, "Echo behavior in large populations of chemical oscillators." Phys. Rev. X 6, 041054 (2016). 


\title{
Chapter Four
}

\begin{abstract}
The phase response curve (PRC) of an oscillator is useful in determining the entrainment mechanisms of oscillators perturbed by external stimuli. To characterize the PRC of a BelousovZhabotinsky (BZ) reaction system, we vary the initial concentration of bromate or bromide in the BZ solution. We report on phase-locked synchronization and switching patterns in a pair of coupled photosensitive BZ oscillators. The oscillators, $\mathrm{Ru}(\mathrm{bpy})_{3}^{2+}$-loaded beads in catalyst-free BZ solutions, were perturbed with light pulses. We quantify the transient changes by constructing the PRC from the resultant oscillations. The PRCs measure the effect of an illumination pulse on the phase of a BZ oscillator. Depending on the phase at which the pulse is applied and the chemical composition of the BZ solution, the oscillations are either advanced or delayed relative to the unperturbed oscillations. We present both experimental measurements and numerical simulations of the dynamical behavior of a pair of photochemically coupled BZ oscillators. An analysis of the behavior reveals phase-locked oscillations and periodic and aperiodic switching of oscillations from in-phase to out-of-phase synchronization.
\end{abstract}




\section{Chapter Four}

\subsection{Coupled BZ Chemical Oscillators}

The Belousov-Zhabotinsky (BZ) reaction exhibits a rich variety of dynamical behaviors that are similar to those found in biological systems, such as fireflies flashing in unison, rhythmic beating of cardiac pacemaker cells, and central pattern generators that control rhythmic actions. The dynamical behaviors of the organisms, cells, and neurons in these systems show remarkable self-organization and synchronization as a result of their interactions. Synchronization patterns of neurons in the brain are linked to diseases such as epilepsy, schizophrenia, and autism [1]. To understand the collective behavior of coupled oscillators, we need to understand how the individual oscillators respond to external stimuli. The sensitivity of the oscillators to external stimuli can be characterized by their Phase Response Curves (PRC) [25]. A PRC is a graphical representation that quantifies the change of the oscillator phase as a function of the phase at which the stimulus was applied.

In this chapter, we investigate the photochemical responses induced by light on BZ oscillators with two aims. The first aim is to characterize the PRCs in the photosensitive $\mathrm{Ru}(\mathrm{bpy}){ }_{3}^{2+}{ }^{+}$-catalyzed $\mathrm{BZ}$ reaction as a function of the $\mathrm{BZ}$ reaction mixture composition. The second aim is to examine the effect of the phase responses on the synchronization pattern of a pair of photochemically coupled BZ oscillators. The effect can be studied for various excitatoryinhibitory regimes and varying natural period heterogeneity. We report experimental results, followed by numerical results obtained using a modified ZBKE model $[15,19]$ described in Subsection 2.3.2. We describe phase-locked synchronization and switching patterns of the coupled oscillators. A parameter plot will demonstrate the influence of the PRC and heterogeneity of natural periods on the entrainment patterns of the coupled BZ oscillators at varying coupling strength. 


\subsection{Phase Response Curve: Photosensitive $\mathrm{Ru}(\mathrm{bpy})_{3}^{2+}$-Catalyzed BZ Oscillators}

The response of an oscillator to external stimuli such as a light pulse, composition change, or temperature variation can be quantified using a PRC. The PRC is widely used to study the response of dynamical systems to external stimuli in various fields, such as physiological rhythms, circadian rhythms, and neuroscience [2-5]. In the photosensitive BZ system, the periodic oscillation in a $\mathrm{Ru}(\mathrm{bpy})_{3}{ }^{2+}$-catalyzed $\mathrm{BZ}$ oscillator can be altered by applying a light pulse [11-18]. The phase shift in the BZ oscillator can be characterized with respect to reaction mixture composition and the phase of the oscillator when the perturbation is applied. To measure and characterize the phase responses of the BZ reaction oscillations due to perturbation by a light pulse, we construct a PRC and a phase transition curve (PTC). The PTC is presented in the form of a new phase relative to the old phase [6]. The old phase refers to the phase at the time of perturbation and the new phase denotes the phase at the perturbation plus the phase shift. The transient changes measured by PRC and PTC plots can aid in understanding the response properties of oscillating BZ systems to light pulse perturbations. The shape of the PRC can be useful in linking the dynamics of individual oscillators to network dynamics.

To characterize the influence of a light pulse on BZ reaction oscillations in our experimental setup, a sequence of three 1.5-second pulses, separated by 1.3 seconds was applied to the beads at different phases, $\phi(t)$, according to Eq. (4.1). Prior to each light perturbation, the oscillators are allowed to oscillate for multiple cycles without perturbation, and the periods of these unperturbed oscillations are used in the calculation of the natural periods $\left(T_{0}\right)$. After each light perturbation, the phase of the oscillators will shift. The phase shifts, $\Delta \phi(\mathrm{t})$, were calculated as the difference between the periods without $\left(T_{0}\right)$ and with $\left(T_{1}\right)$ light perturbation, as shown in Eq. (4.2). The periods of the unperturbed BZ reaction oscillations are relatively stable until a light perturbation is applied, at which time the oscillation period either decreases or increases, as illustrated in Figure 4.1. 

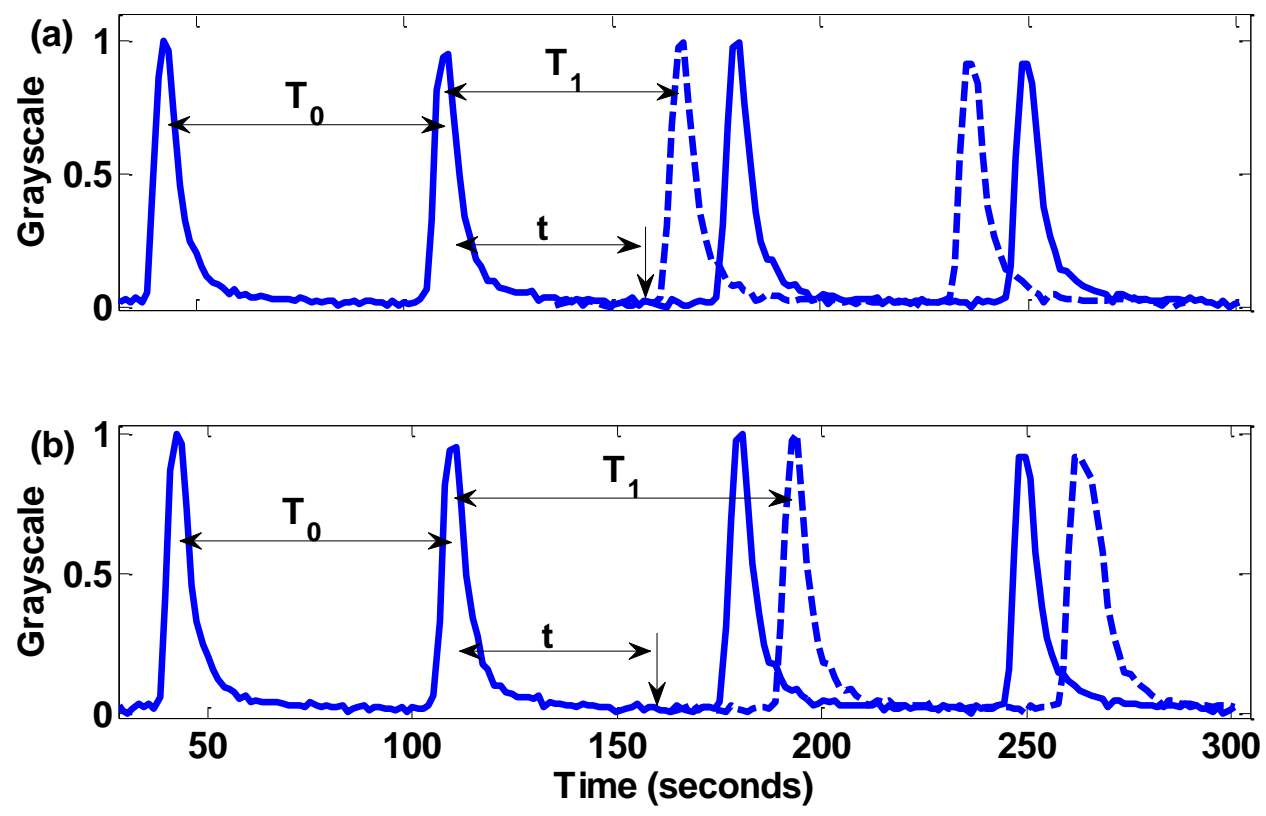

Figure 4.1: Time series plots showing phase shifts (phase advancing and phase delaying). (a) After perturbation, the period of oscillation decreases, and (b) after perturbation, the period of oscillation increases. The solid blue line shows the oscillations with the natural period, while the dotted blue line shows the course of oscillations after a perturbation at the point shown by the vertical arrow after time $\mathrm{t}$ from the previous oscillation peak.

The effect of a light perturbation on the BZ system can be explained via the production of activator and/or inhibitor in the BZ system [7-8]. The illustration in Figure 4.1(a) shows a phase advance in the photoexcitatory regime, which shortens the oscillation period after perturbation. Here, the light pulse excites the reduced form of the catalyst, and the excited catalyst, $\mathrm{Ru}(\mathrm{bpy})_{3}^{2+*}$, reacts directly with the reactant $\mathrm{BrO}_{3}^{-}$, and in a series of subsequent reactions produces the autocatalytic species, $\mathrm{HBrO}_{2}$. Additional $\mathrm{HBrO}_{2}$ typically results in a phase advance, as shown in Figure 4.1(a), although it has little effect during the excited or recovery period of the oscillation [15]. Figure 4.1(b) shows a case of phase delay in the photoinhibitory regime of the BZ reaction. After generation of the excited form of the reduced catalyst, the excited catalyst reacts directly with bromomalonic acid to produce the inhibitor, $\mathrm{Br}^{-}$. The production of $\mathrm{Br}^{-}$results in a phase delay as illustrated by Figure 4. 1(b).

In our experimental investigation of the photosensitive BZ system, we perturbed the Rucatalyzed beads using illumination from a SLM. The examples in Figure 4.1(a-b) show perturbations at a particular phase of the limit cycle, and demonstrate how the oscillator 
responds to the perturbation. However, the oscillator response might have a different magnitude if it is perturbed at a different phase. The phase of the oscillator at which the stimuli is applied, can be calculated by Eq. (4.1)

$$
\phi(t)=\frac{2 \pi t}{T_{0}} .
$$

$\mathrm{T}_{\mathrm{o}}$ is the unperturbed period, corresponding to the oxidation of the $\mathrm{Ru}(\mathrm{bpy})_{3}^{2+}$ period, and $\mathrm{t}$ is the perturbation time from the reference point, which is the previous peak of oscillation. The reference point is defined to be zero when the oscillation cycle reaches the peak. To get a complete picture of how the oscillator responds to perturbations at different phases, the phase shift at these phases is determined using Eq. (4.2)

$$
\Delta \phi(\mathrm{t})=\frac{2 \pi\left(\mathrm{T}_{0}-\mathrm{T}_{1}\right)}{\mathrm{T}_{0}}
$$

where $T_{1}$ is the period of the cycle with the perturbation. The new phase for constructing the PTC is given by

$$
\phi_{\text {new }}(\mathrm{t})=\phi(\mathrm{t})+\Delta \phi(\mathrm{t}) .
$$

The transient change of the phase of an oscillator is quantified by comparing the period difference between perturbed and unperturbed cycle. If $\Delta \phi<0$, the oscillation period lengthens after the perturbation, and this phenomenon is called phase retardation or phase delay. If $\Delta \phi>$ 0 , the oscillation period shortens after the perturbation, and this phenomenon is called phase advance. If $\Delta \phi=0$, there is no phase shift, and this usually occurs during the refractory phase of the $\mathrm{BZ}$ reaction.

The experiments were carried out using the experimental method described in chapter two. We determine the phase responses of $\mathrm{Ru}(\mathrm{bpy})_{3}^{2+}$-catalyzed $\mathrm{BZ}$ oscillators to light perturbations using forty to sixty beads dispersed in a Petri dish. The beads were perturbed at the grayscale value of 250 in a sequence of three pulses separated by 1.3 seconds, for a total of 4.5 seconds of illumination time. Five perturbations where applied during each experiment in an interval that allows at least three oscillation cycles for determining the natural periods. The recorded data are analyzed using MATLAB and calculations are carried out using Eqs. (4.1) - (4.3) to plot the PRC and PTC. 


\subsection{Experimental Results}

\subsubsection{Phase Response Curve and Phase Transition Curve}

In the experimental results, time series plots of the chemical oscillators' normalized transmitted light intensities are used to extract the periods used in the calculation of phases and phase shifts. In general, the shapes of the PRC and PTC shown below depend on the dynamics that characterize the perturbed oscillators. The phase and BZ chemical composition influences the magnitude of the phase shift and causes either oscillation phase delay or phase advance. The character of the PRC changes from predominantly excitatory to predominantly inhibitory depending on the chemical composition. Thus, the PRC shapes are measured as bromate and bromide concentrations are varied. To characterize the phase response of a wide regime of these $\mathrm{Ru}(\mathrm{bpy})_{3}^{2+}$-catalyzed $\mathrm{BZ}$ oscillators to light pulses, we varied the chemical composition of the BZ solution as specified below.

\subsubsection{The Effect of Bromate Concentration in the BZ Reaction Mixture}

In the experimental results shown below, we used a reaction mixture with the following concentrations: $[\mathrm{MA}]=0.096 \mathrm{M},\left[\mathrm{H}_{2} \mathrm{SO}_{4}\right]=0.78 \mathrm{M},\left[\mathrm{Br}^{-}\right]=0.060 \mathrm{M}$, and varied $\left[\mathrm{BrO}_{3}^{-}\right]$. In a 25.0 $\mathrm{mL}$ or $50.0 \mathrm{~mL}$ volumetric flask the reagents were added in the following order: malonic acid (MA), sodium bromate $\left(\mathrm{NaBrO}_{3}\right)$, sulfuric acid $\left(\mathrm{H}_{2} \mathrm{SO}_{4}\right)$, and sodium bromide $(\mathrm{NaBr})$. The addition of the bromide turned the solution to a brown color due to the production of bromine. After a few minutes, the brown color disappeared and the solution became colorless due to the bromination of malonic acid. Water was then added to the volumetric flask to the mark. The reaction mixture was tuned by varying the bromate concentration so that the BZ reaction changed from a predominantly inhibitory regime to a predominantly excitatory regime.

The bromination of MA during the induction period establishes the actual initial concentrations. The formation of BrMA follows these stoichiometric equations:

$$
\begin{gathered}
\mathrm{BrO}_{3}^{-}+5 \mathrm{Br}^{-}+6 \mathrm{H}^{+} \rightleftarrows 3 \mathrm{Br}_{2}+3 \mathrm{H}_{2} \mathrm{O} \\
\left(\mathrm{MA}+\mathrm{Br}_{2} \rightleftarrows \mathrm{BrMA}+\mathrm{Br}^{-}+\mathrm{H}^{+}\right) \times 3
\end{gathered}
$$

$$
\mathrm{BrO}_{3}^{-}+2 \mathrm{Br}^{-}+3 \mathrm{MA}+3 \mathrm{H}^{+} \rightleftarrows 3 \mathrm{BrMA}+3 \mathrm{H}_{2} \mathrm{O}
$$



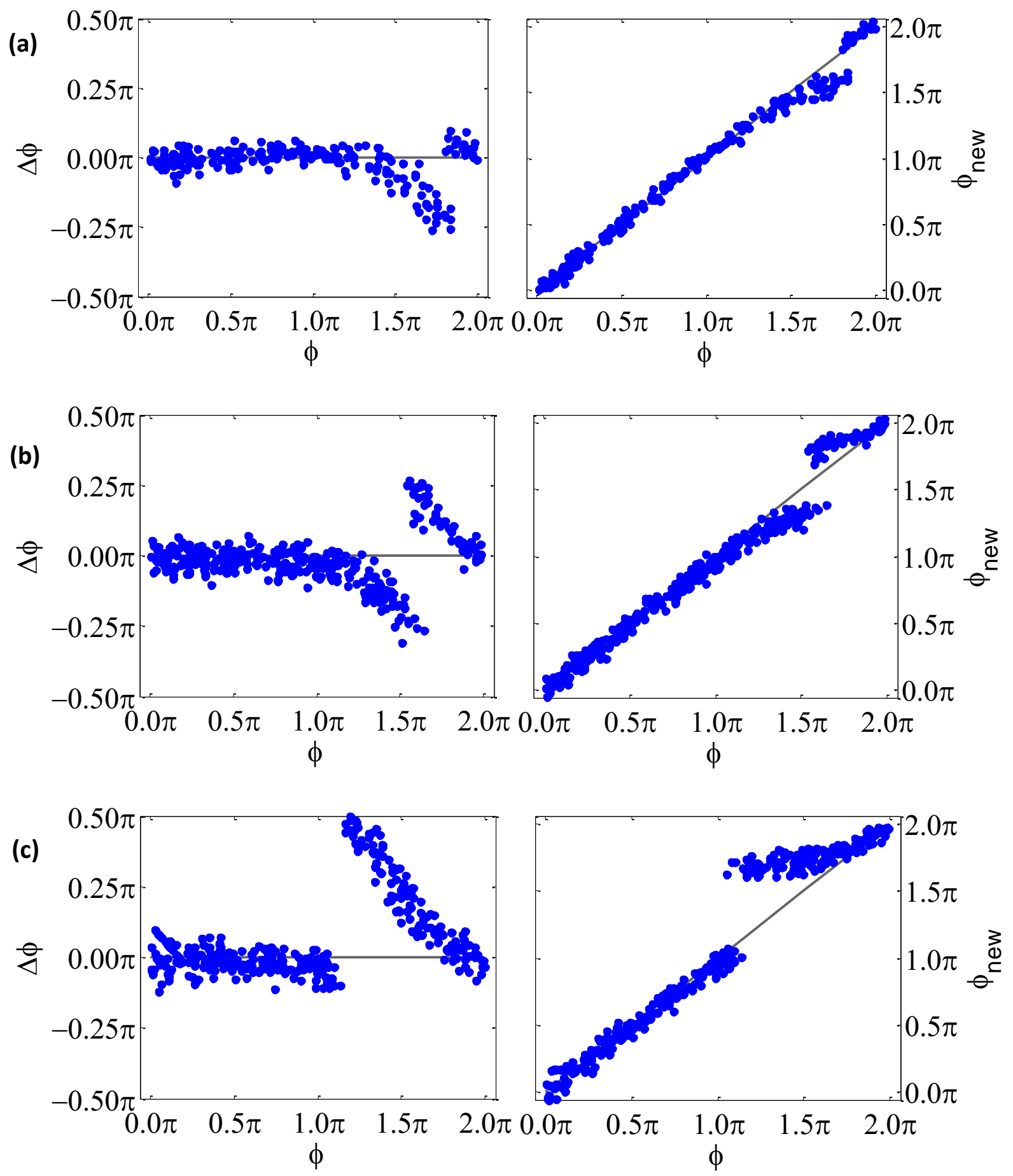

Figure 4.2: Phase response curves for the BZ reaction that show the transition from inhibitory to excitatory regimes as bromate concentration is increased. Experimental PRCs (left) and PTCs (corresponding right) of $\mathrm{Ru}(\mathrm{bpy})_{3}^{2+}$-catalyzed $\mathrm{BZ}$ oscillators. (a) $\left[\mathrm{BrO}_{3}^{-}\right]=0.29 \mathrm{M}$ (b) $\left[\mathrm{BrO}_{3}^{-}\right]=0.41 \mathrm{M}$ (c) $\left[\mathrm{BrO}_{3}^{-}\right]=0.61 \mathrm{M}$. 
Equations (4.4) show the formation of $\mathrm{BrMA}$ from the reaction of $\mathrm{Br}^{-}$with $\mathrm{BrO}_{3}^{-}$in acidic condition to form $\mathrm{Br}_{2}$, which then is followed by the bromination of MA to give BrMA. Using the stoichiometry, the established initial concentrations are: $[\mathrm{MA}]=0.006 \mathrm{M},[\mathrm{BrMA}]=0.090 \mathrm{M}$, $\left[\mathrm{H}_{2} \mathrm{SO}_{4}\right] \approx 0.78 \mathrm{M}$ and varied $\left[\mathrm{BrO}_{3}^{-}\right]$.

\subsubsection{The Effect of Bromomalonic Acid Concentration in the BZ Reaction Mixture}

A catalyst-free BZ solution preparation procedure similar to that described above was used with the following concentrations: $\left[\mathrm{H}_{2} \mathrm{SO}_{4}\right] \approx 0.78 \mathrm{M},\left[\mathrm{BrO}_{3}^{-}\right]=0.44 \mathrm{M}$, and varied $[\mathrm{BrMA}]$. The reaction mixture was tuned by varying the bromomalonic acid concentration so that the BZ reaction changed from a predominantly inhibitory regime to a predominantly excitatory regime, see Figure 4.3.

The PRC shapes of $\mathrm{Ru}(\mathrm{bpy})_{3}^{2+}$-catalyzed $\mathrm{BZ}$ oscillators are influenced by the reaction solution composition, which determines the dominant channel in the photochemical mechanism (see section 2.4.2 for details). A systematic increase in bromate concentration resulted in the PRC shapes to change from a predominantly inhibitory to a predominantly excitatory regime, whereas a systematic increase in bromomalonic acid concentration resulted in the PRC shapes to change from a predominantly excitatory to a predominantly inhibitory regime. Between the extreme ends of the excitatory-inhibitory regimes, the BZ oscillators' PRCs are characterized by both phase advancement and phase delay in different regions of the plot, separated by a sharp transition. A transition from phase delay to phase advance occurs at this point. Increasing bromate concentration in the BZ solution results in a shift to lower phase of the transition in the PRC plot, whereas increasing bromomalonic acid concentration in the BZ solution results in a shift to higher phase of the transition in the PRC plot. 

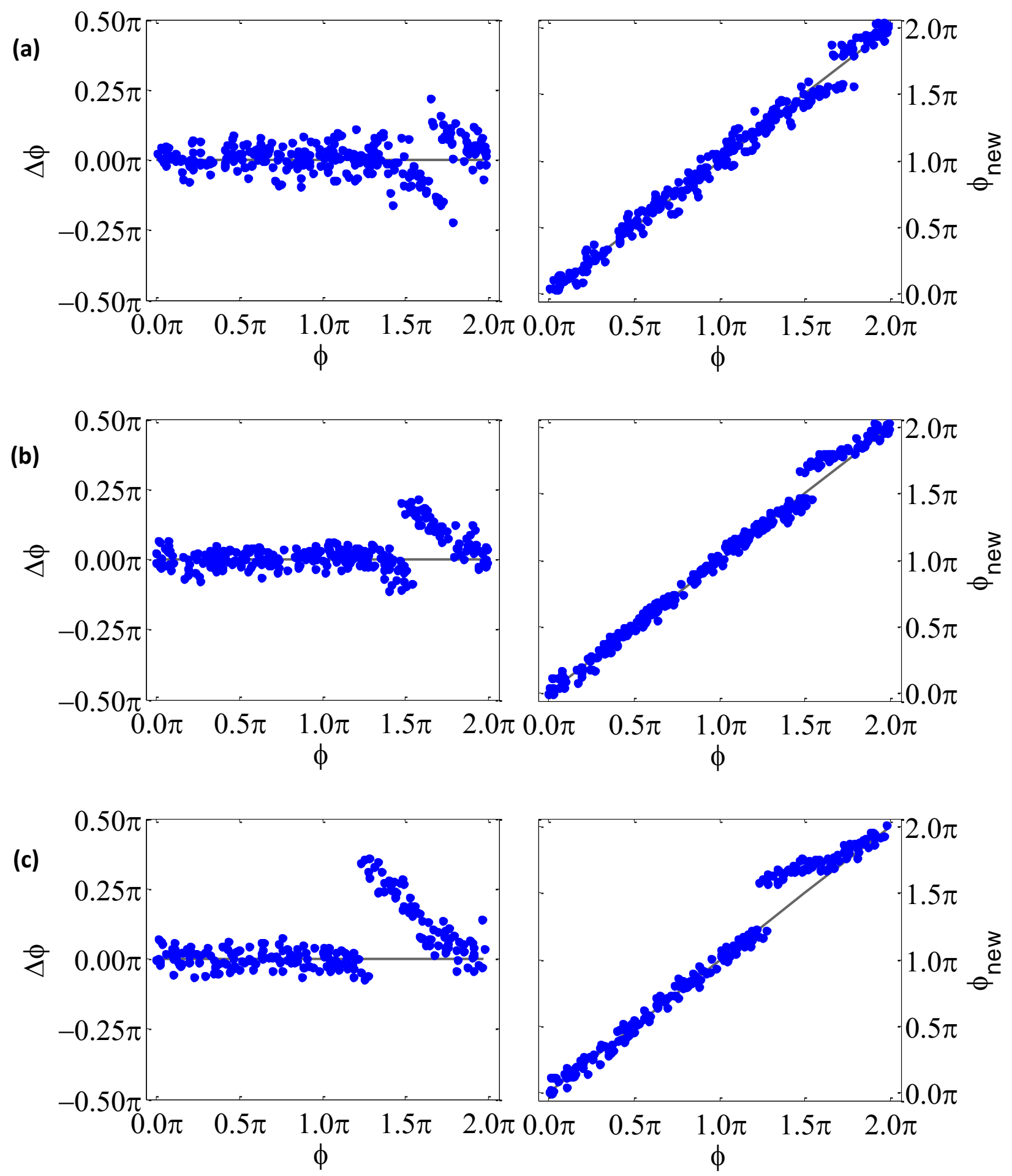

Figure 4.3: PRC and PTC plots that show the transition from inhibitory to excitatory regimes as bromide concentration is decreased. Experimental PRCs (left) and PTCs (corresponding right) of Ru(bpy) ${ }_{3}^{2+}$ catalyzed BZ oscillators. (a) $[\mathrm{BrMA}]=0.096 \mathrm{M},[\mathrm{MA}]=0.000 \mathrm{M},\left[\mathrm{Br}^{-}\right]=0.026$ (b) $[\mathrm{BrMA}]=0.096 \mathrm{M},[\mathrm{MA}]=$ $0.000 \mathrm{M}(\mathrm{c})[\mathrm{BrMA}]=0.03 \mathrm{M},[\mathrm{MA}]=0.066 \mathrm{M}$. 


\subsection{PRC Numerical Simulations Results}

Simulations were based on Eq. (2.22) to determine the PRCs of the oscillators using the perturbation $\phi=0.075$. The simulation results compare well with our experimental observations. Similar to the experimental observation, the simulation results below show the PRC plots when $\alpha$ is varied.

(a)
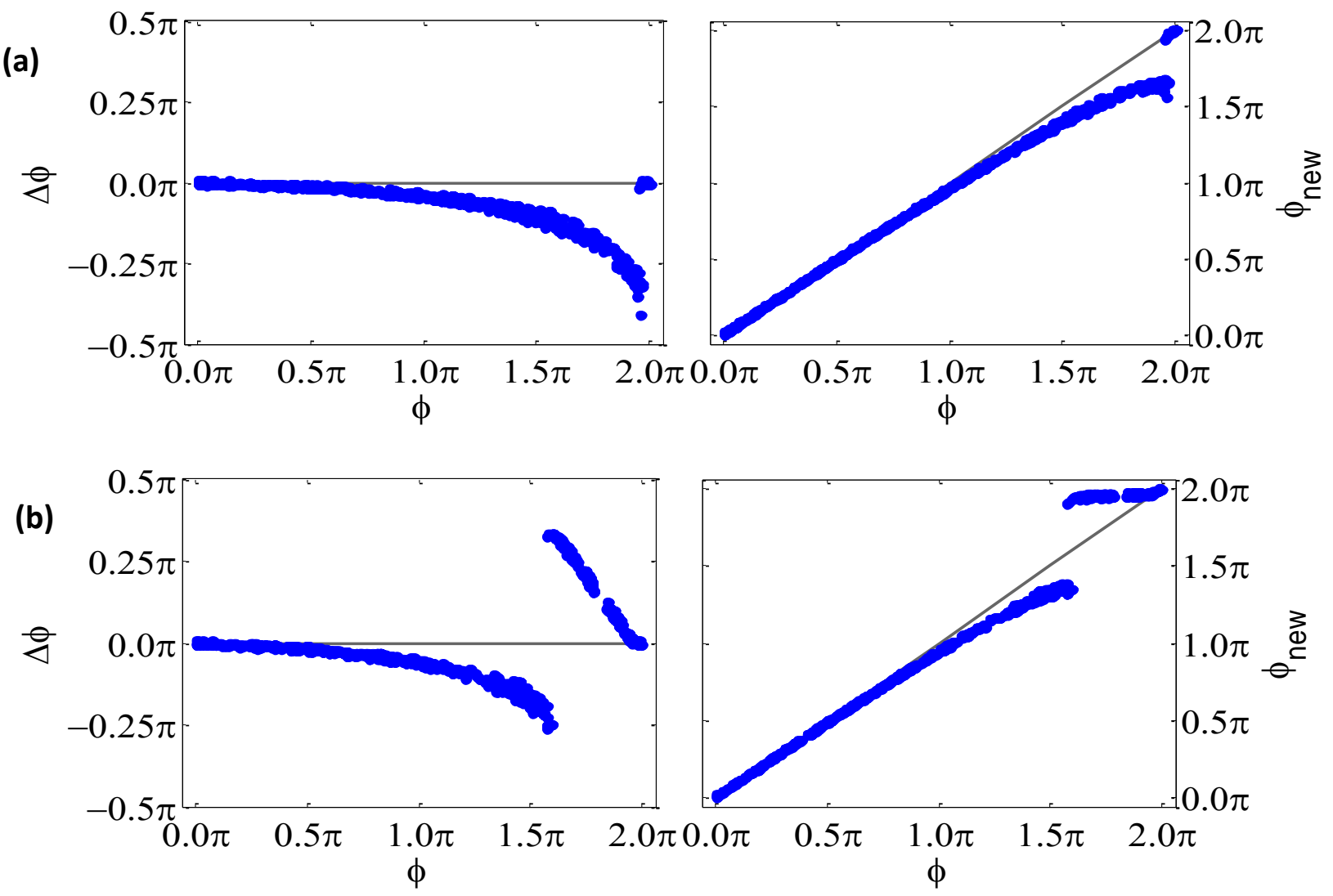

(c)
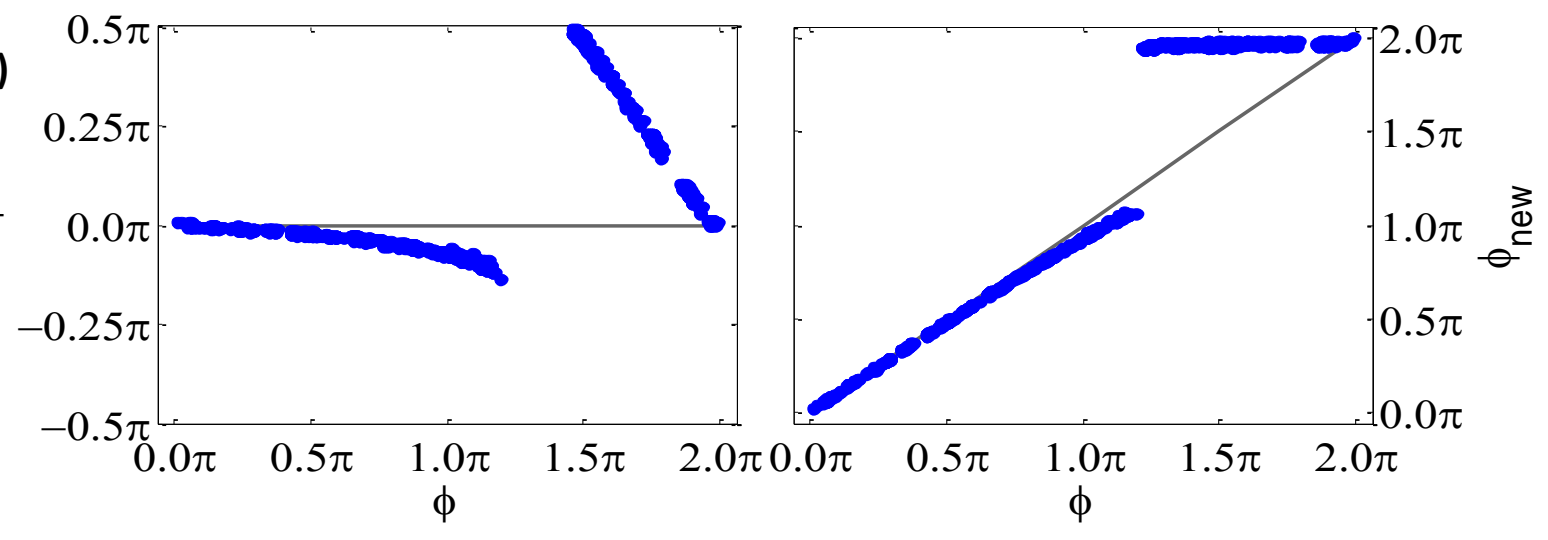

Figure 4.4: Simulations of the PRC and PTC using the modified ZBKE model. The plots show the transition from the inhibitory regime to excitatory regime as $\alpha$ increases. The plots on the left are the PRCs and the plots on the right are the PTCs described by Eqs. (4.1)-(4.3). (a) $\alpha_{0}=0.10$ (b) $\alpha_{0}=0.50$ (c) $\alpha_{0}=0.70$. 
The PRC of an oscillator can be used as a guide to gain insights into the entrainment mechanism, to classify oscillator responses, and it is helpful in understanding or predicting the collective behaviors in coupled pairs of oscillators. After characterizing the effects of varying the BZ reaction mixture composition from the excitatory to the inhibitory regime, we can use the different regimes to implement photochemical coupling in a network of Ru-catalyzed BZ oscillators.

\subsection{Coupling Pair of BZ Oscillators}

Interacting oscillatory systems, such as yeast cells or BZ oscillators, tend to exhibit a collective rhythm. The interactions occur by mutual signaling in the form of stimuli that alters the behavior of the oscillators. Two coupled oscillators can exhibit a variety of dynamical behaviors, ranging from synchronization to aperiodic oscillations [9-10]. Here, we examine the effect of the parameters such as the heterogeneity in period, quantified here as period ratio of the two oscillators, the excitatory-inhibitory regimes, and the coupling strength on the dynamical behavior of coupled BZ oscillators. The aim is to characterize the influence of the period heterogeneity and the inhibitory-excitatory regimes on the synchronization patterns of two coupled BZ oscillators. We quantify the synchronization patterns using the phase lag and the periods of the two oscillators during coupling. We begin by presenting experimental results describing phase-locked synchronization patterns followed by switching patterns in a pair of coupled BZ oscillators. We explore further the effect of frequency (period) heterogeneity and the excitatory-inhibitory regimes on the observed experimental synchronization behavior in simulations of coupled BZ oscillators. The simulations reveal four regions of synchronization patterns: (i) exclusively in-phase synchronization, (ii) exclusively out-of-phase synchronization, (iii) bistable with coexisting in-phase and out-of-phase synchronization, which depends on initial phase conditions, and (iv) switching patterns with periodic and "aperiodic" switching.

In the experimental setup, two BZ oscillators were coupled bidirectionally for various BZ reaction mixture compositions and heterogeneous period distributions. The oscillators are coupled using the difference of their normalized grayscale values, $\left(Z_{i}-Z_{j}\right)$. The feedback is calculated by Eq. (3.3). The interaction between the two oscillators is illustrated in Figure 4.5. 

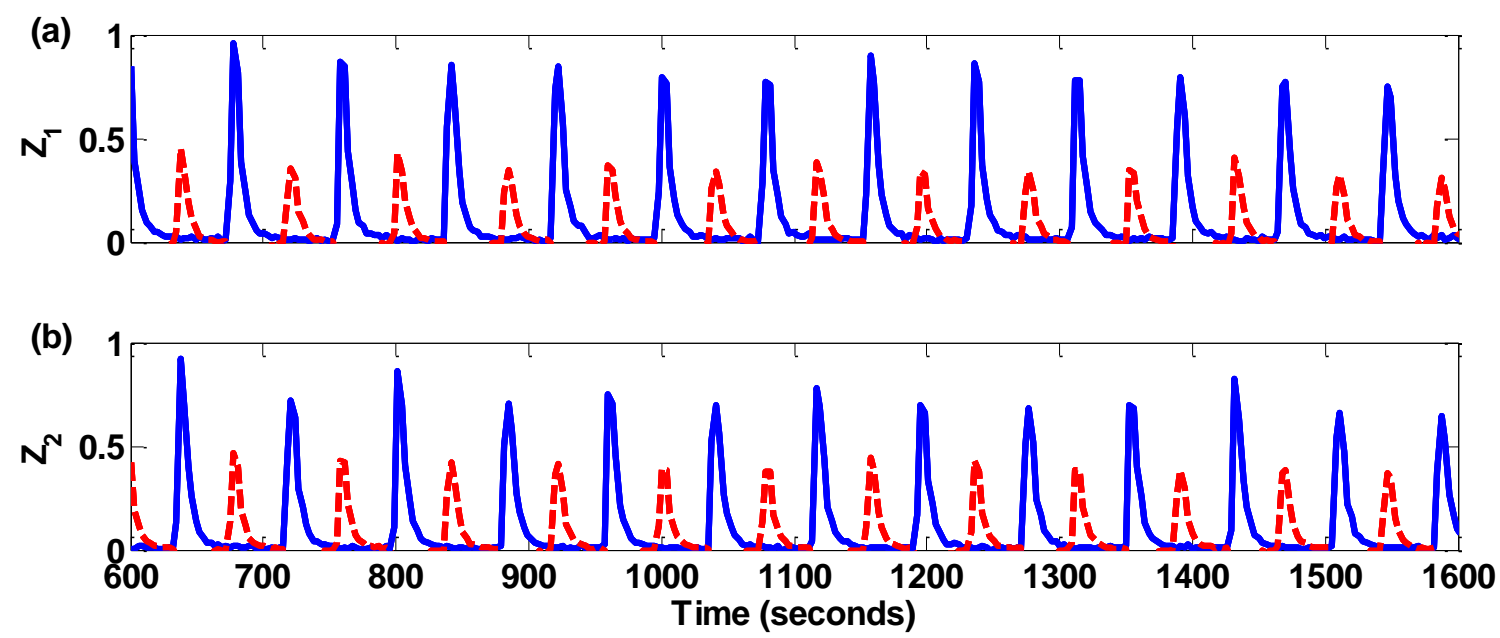

Figure 4.5: Time series (blue line) showing oscillations of interacting oscillators (1) and (2). The interaction signals are shown by the dotted red line.

The normalized grayscale values $Z_{i}$ and $Z_{j}$ for oscillators $i$ and $j$ are obtained from the transmitted light intensity of the oscillators, which is proportional to the concentration of $\mathrm{Ru}(\text { bipy })_{3}{ }^{3+}$.

In the experimental plots shown in Figure 4.6, a BZ system in a mixed excitatory-inhibitory regime was used to obtain the observed synchronization patterns. The BZ solution composition produces a PRC with both inhibitory and excitatory region has the following concentrations: $[\mathrm{MA}]=0.006 \mathrm{M},[\mathrm{BrMA}]=0.09 \mathrm{M},\left[\mathrm{BrO}_{3}^{-}\right]=0.45 \mathrm{M}$, and $\left[\mathrm{H}_{2} \mathrm{SO}_{4}\right] \approx 0.78 \mathrm{M}$.

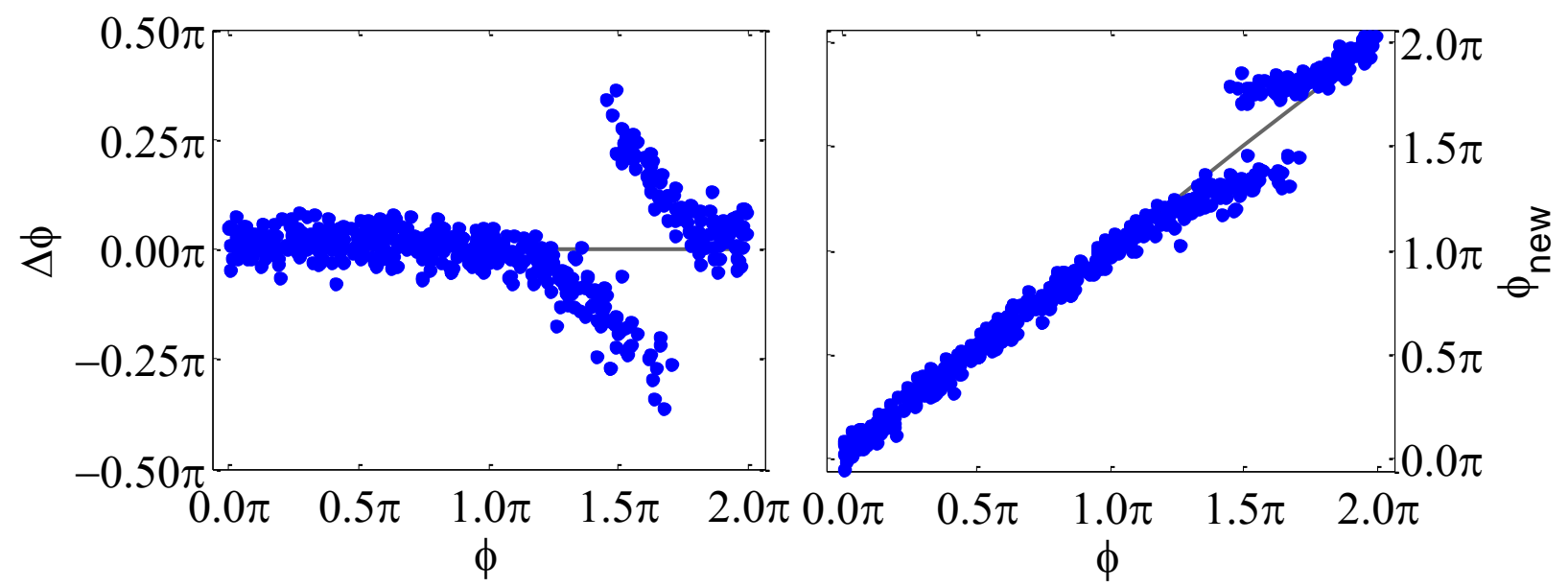

Figure 4.6: PRC and PTC plots for coupled BZ oscillators with competing inhibitory and excitatory regimes. 
The behavior between the two oscillators can be quantitatively described by calculating the phase lag (also corresponding to perturbation phase) and the oscillations periods. The phase of the oscillator at the time of the perturbation is calculated using

$$
\phi_{i}=2 \pi \frac{\delta t}{T_{o i}},
$$

where $\phi_{i}$ is the perturbation phase, $\delta t$ is the time difference between the two oscillators' peaks, and $T_{o i}$ is the natural period for oscillator $i$.

The following results highlight the influence of heterogeneity and the PRC at a given coupling strength, $k=10$. Under the experimental conditions, we observed phase-locked synchronization and switching synchronization patterns. Phase-locked synchronization describes both in-phase and out-of-phase synchronization, where a constant phase difference is maintained during the interaction of the oscillators. In the switching synchronization patterns, the pair of oscillators alternate between in-phase and out-of-phase during the interaction of the oscillators. The results of the corresponding simulations producing switching synchronization patterns are shown below, and it is followed by a numerical analysis. In the experimental work, we can adjust the coupling strength and BZ solution composition; however, the natural periods and initial phase difference cannot be adjusted. The effect of the initial phase difference between the oscillators and the heterogeneous period distributions on the dynamical behaviors can be addressed using numerical simulations.

\subsubsection{Phase-locked Synchronization}

The experimentally measured gray level for two oscillators as a function of time is show in Figures (4.7) and (4.8). After the coupling is switched on at approximately 500 seconds, the two oscillators begin to synchronize, and the oscillators fire with a constant phase difference of approximately 180 degrees for out-of-phase synchronization. For in-phase synchronization, the oscillators fire nearly simultaneously. In order to quantify and classify the synchronization patterns, we plot the periods and the perturbation phases when each oscillator receives a light pulse as a function of time, as shown in Figures 4.7 and 4.8. The phase-locked synchronization is characterized by a constant phase lag and similar periods. 
(a)

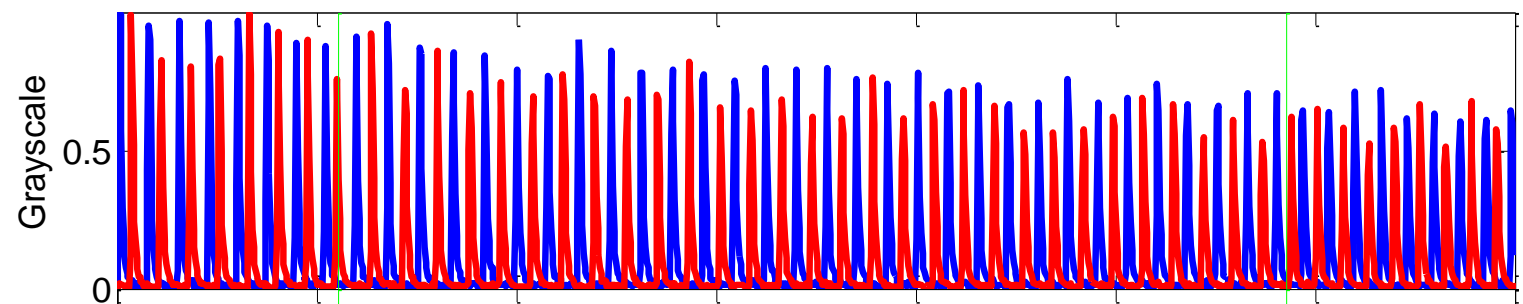

(b)

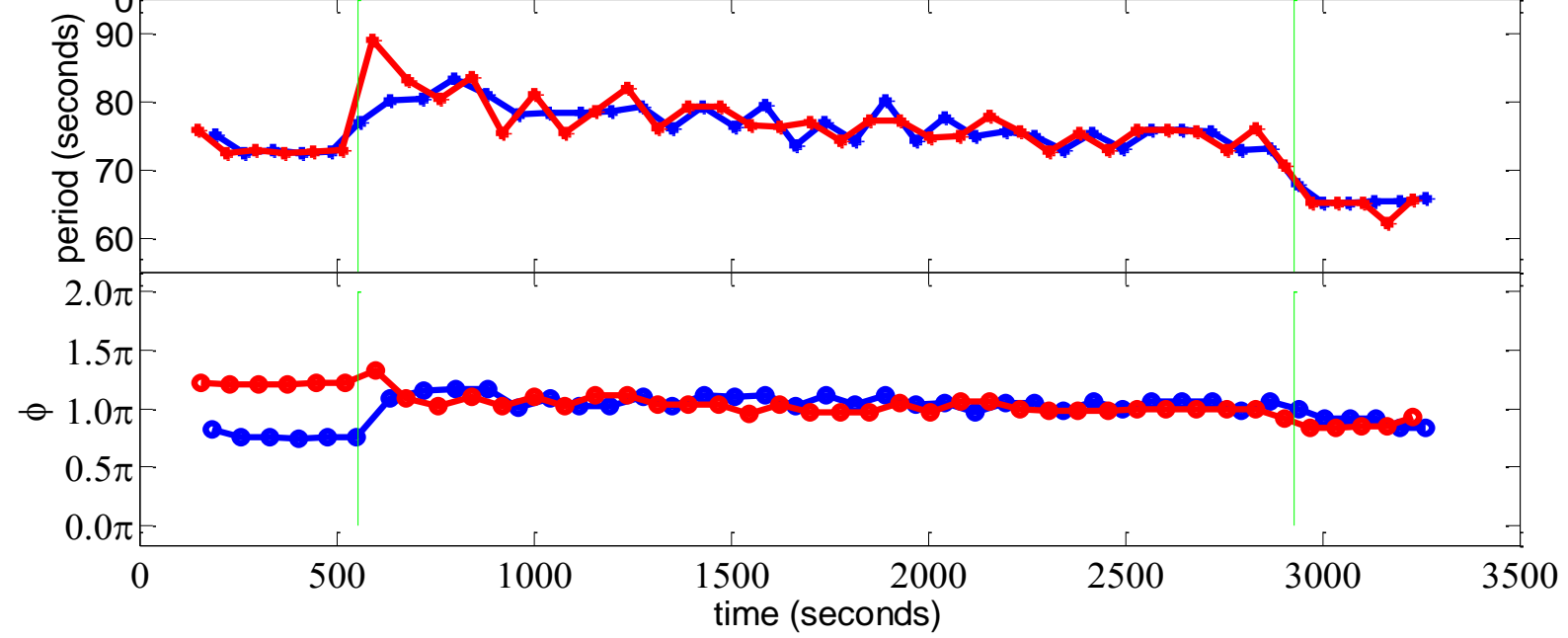

Figure 4.7: A pair of coupled Ru-catalyzed BZ oscillators, displaying anti-phase synchronization: (a) time series, (b) calculated periods, and (c) perturbation phases. Coupling is turned on after 500 seconds and turned off close to 3000 seconds; the experiment runs for approximately one hour. Coupling is applied between the green lines. Initial concentrations: $[\mathrm{MA}]=0.006 \mathrm{M},[\mathrm{BrMA}]=0.09 \mathrm{M},\left[\mathrm{BrO}_{3}^{-}\right]=0.45 \mathrm{M}$, and $\left[\mathrm{H}_{2} \mathrm{SO}_{4}\right] \approx 0.78 \mathrm{M}$.

For out-of-phase oscillations, the phase difference between the two oscillators is close to 180 degrees, which is within the inhibitory phase region in Figure 4.6. The inhibitory effect from perturbations received by each oscillator at the phase shown in Figure 4.7 (c) leads to each oscillator entraining the other oscillator. The natural periods of the two oscillators are nearly the same before and after the coupling is on; the periods of both oscillators increase during perturbation when coupling is on. This is because the perturbation phase falls on the inhibitory region of the phase response curve, and therefore causes a phase delay. 
(a)

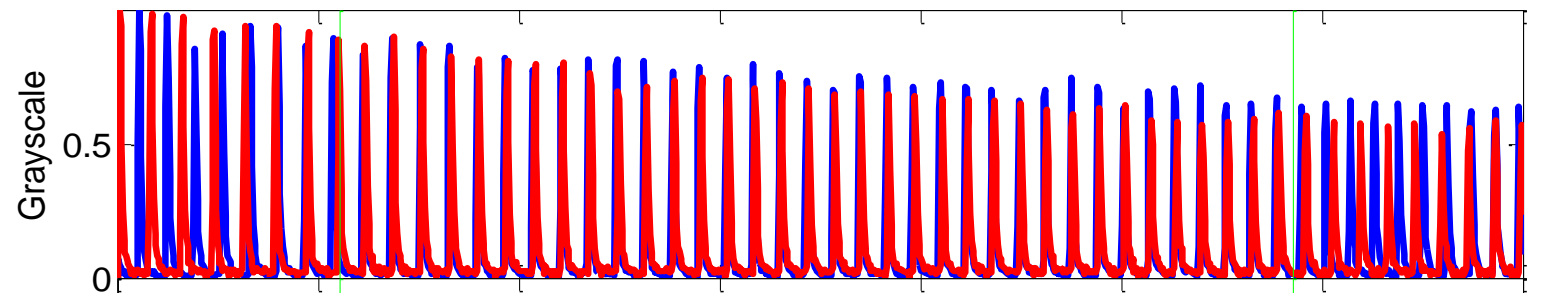

(b)
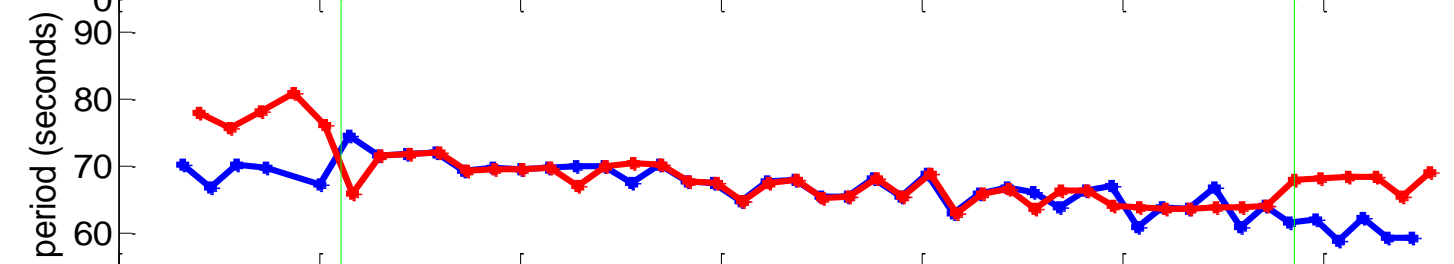

$2.0 \pi$

$1.5 \pi$

(c)

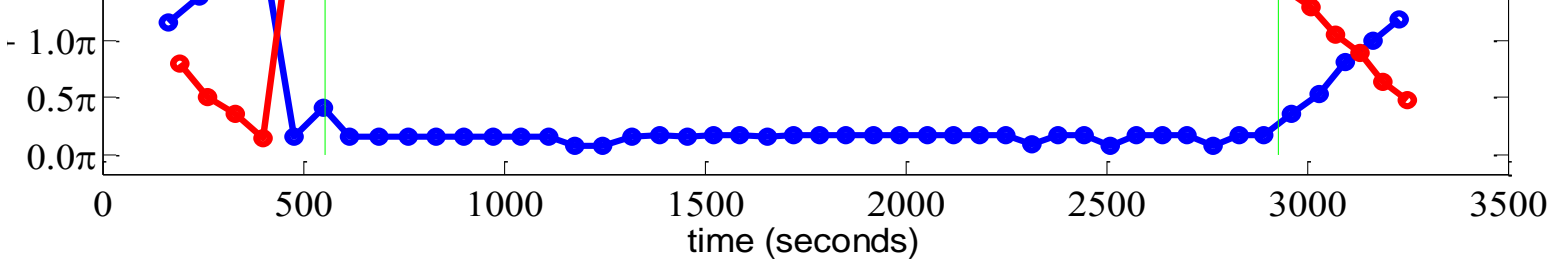

Figure 4.8: A pair of coupled Ru-catalyzed BZ oscillators, displaying in-phase synchronization: (a) time series, (b) calculated periods, (c) perturbation phases. Coupling is applied between the green lines. Initial concentrations: $[\mathrm{MA}]=0.006 \mathrm{M},[\mathrm{BrMA}]=0.09 \mathrm{M},\left[\mathrm{BrO}_{3}^{-}\right]=0.45 \mathrm{M}$, and $\left[\mathrm{H}_{2} \mathrm{SO}_{4}\right] \approx 0.78 \mathrm{M}$.

For in-phase oscillations, the phase difference between the two oscillators is close to 0 degrees, and places the perturbation phase for one oscillator in the excitatory phase region. The oscillator with the higher frequency drives the oscillations. Even though the natural periods of the oscillators are different, when coupling is switched on, the periods of both oscillators become nearly the same and close in length to the natural period of the oscillator with the higher frequency. This can be attributed to the perturbation phase falling in the excitatory region of the phase response curve. The oscillator with the higher frequency acts as the pacemaker driving the oscillations.

\subsubsection{Autonomously Switching Pattern}

In addition to the phase-locked synchronization, we also observe switching patterns. In switching patterns, the synchronization shifts between the two phase-locked synchronization patterns. The time trace of the perturbation phases shows that the phase lag of the two 
oscillators is not constant. The drift in phase lag corresponds to the perturbation alternating between the excitatory and inhibitory regions in the phase response curve, shown in Figure 4.6. The switching pattern from in-phase to out-of-phase and vice versa is likely caused by an attractor (chain recurrent) which passes through the sharp transition in the PRC. The attractor is a function of initial phase difference and the difference in period or heterogeneity between the two oscillators. This results in perturbations moving into either the excitatory or inhibitory regions.

(a)

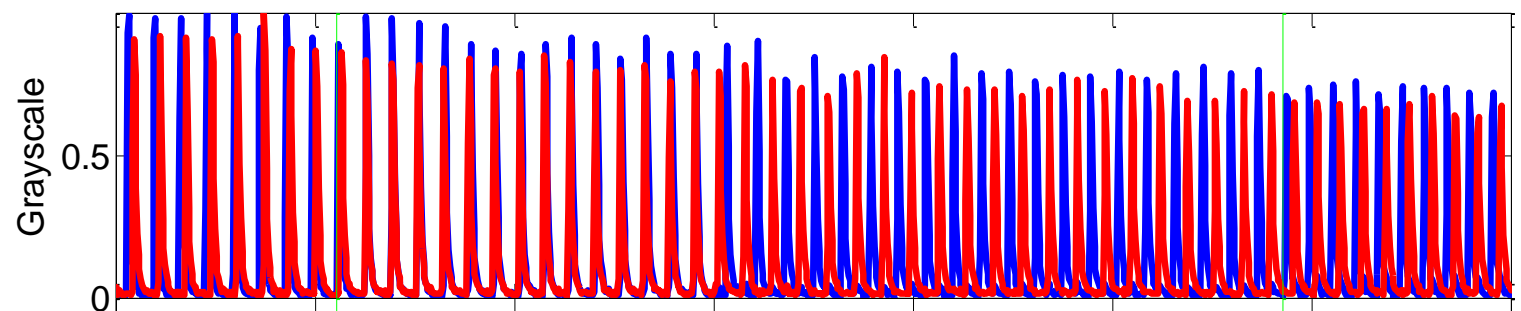

(b)

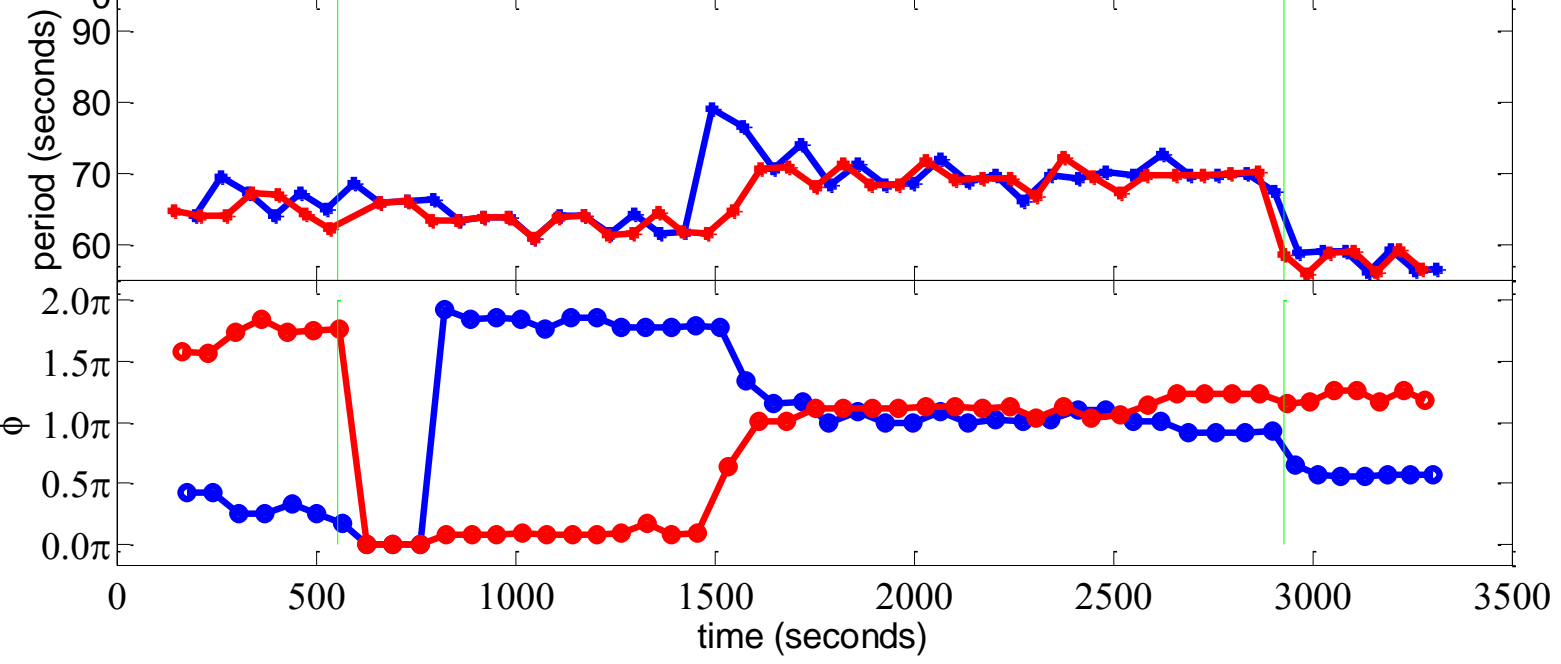

Figure 4.9: A pair of coupled Ru-catalyzed BZ oscillators displaying switching patterns from in-phase to out-of-phase synchronization: (a) time series, (b) calculated periods, and (c) perturbation phases. Coupling is applied between the green lines. Initial concentrations: $[\mathrm{MA}]=0.006 \mathrm{M},[\mathrm{BrMA}]=0.09 \mathrm{M}$, $\left[\mathrm{BrO}_{3}^{-}\right]=0.45 \mathrm{M}$, and $\left[\mathrm{H}_{2} \mathrm{SO}_{4}\right] \approx 0.78 \mathrm{M}$.

The synchronization switches from in-phase to out-of-phase because the perturbation phase shifts to the inhibitory phase region, which is shown by the initial higher period jump. 


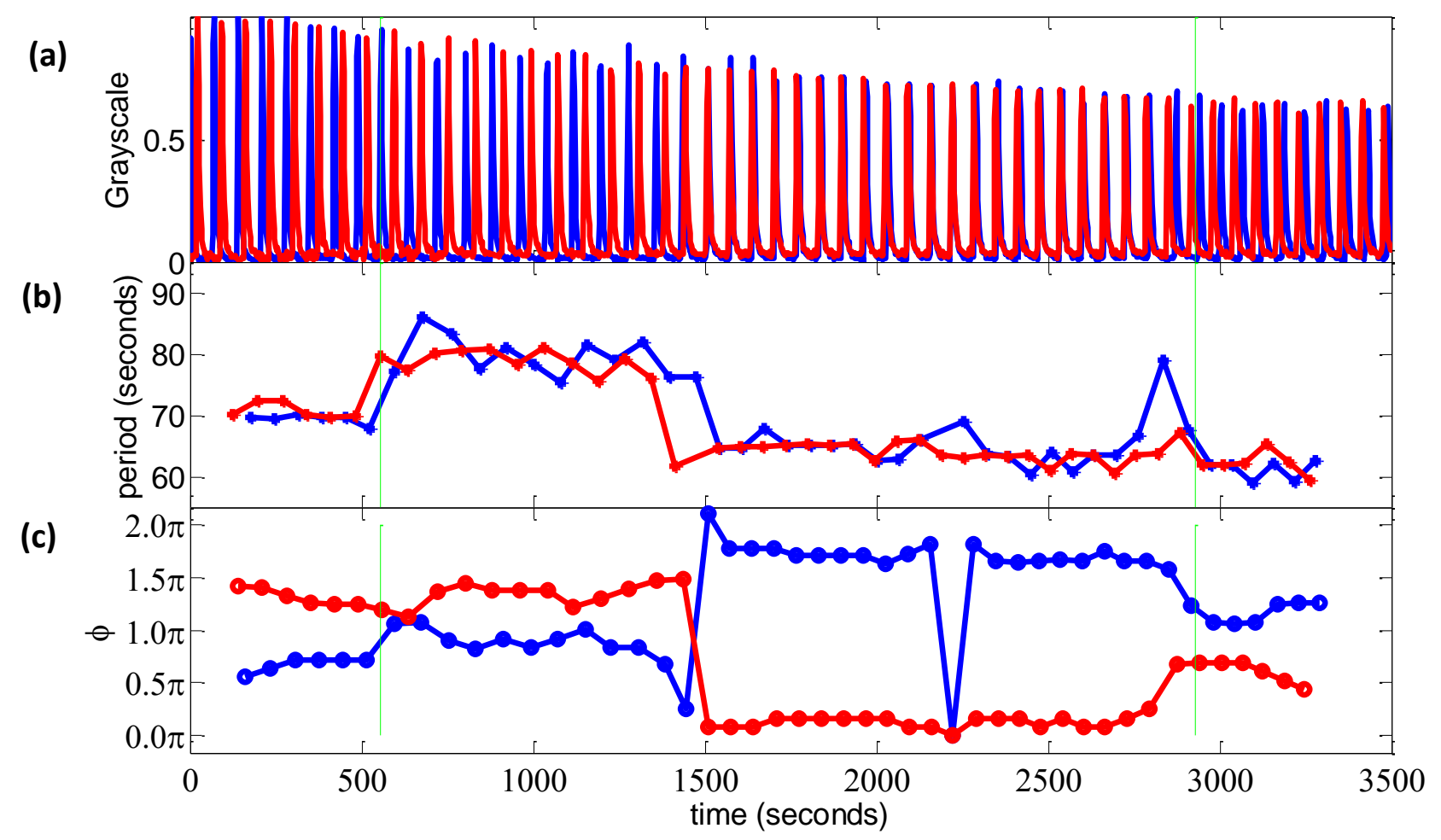

Figure 4.10: A pair of coupled Ru-catalyzed BZ oscillators displaying switching patterns from out-ofphase to in-phase synchronization: (a) time series, (b) calculated periods, and (c) perturbation phases. Coupling is applied between the green lines. Initial concentrations: $[\mathrm{MA}]=0.006 \mathrm{M},[\mathrm{BrMA}]=0.09 \mathrm{M}$, $\left[\mathrm{BrO}_{3}^{-}\right]=0.45 \mathrm{M}$, and $\left[\mathrm{H}_{2} \mathrm{SO}_{4}\right] \approx 0.78 \mathrm{M}$.

The synchronization switches from out-of-phase to in-phase because the perturbation phase drifts into the excitatory phase region. For the inhibitory perturbation phases, successive cycles result in perturbations at different phases of the excitatory-inhibitory regime. In this particular case, this results in drifting of the perturbations into the excitatory region. 
(a)

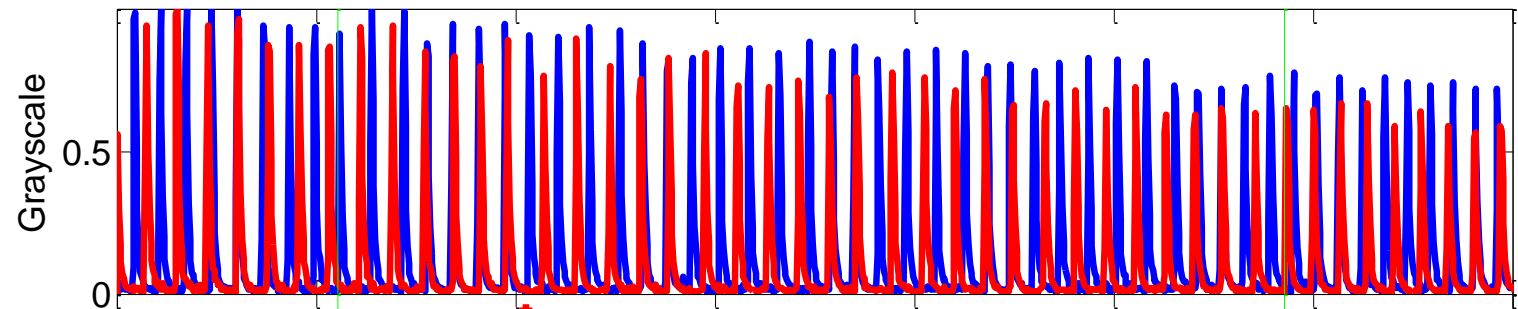

(b)

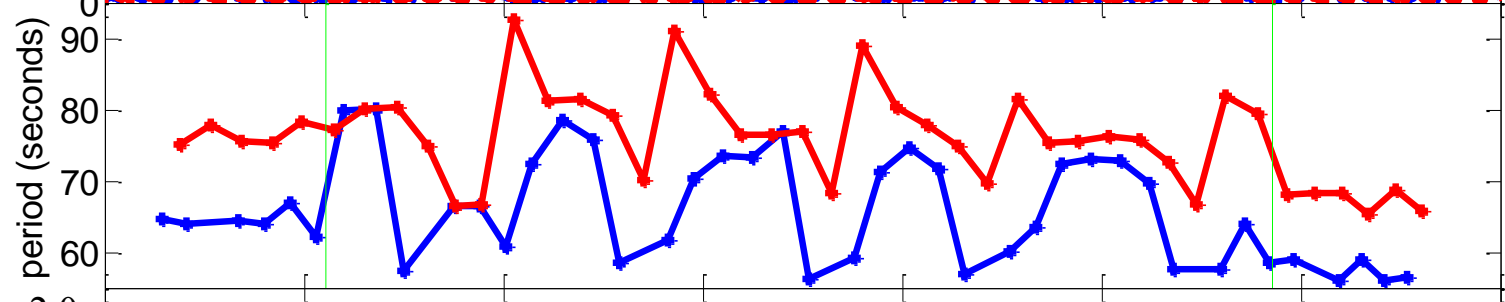

(c)

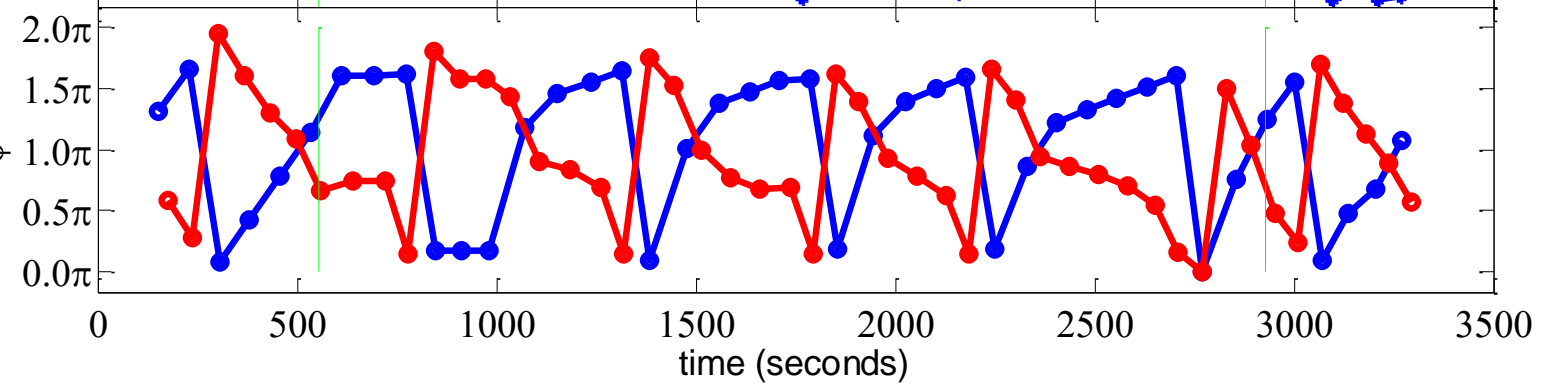

Figure 4.11: A pair of coupled Ru-catalyzed BZ oscillators displaying switching synchronization: (a) time series, (b) calculated periods, and (c) perturbation phases. Coupling is applied between the green lines. Initial concentrations: $[\mathrm{MA}]=0.006 \mathrm{M},[\mathrm{BrMA}]=0.09 \mathrm{M},\left[\mathrm{BrO}_{3}^{-}\right]=0.45 \mathrm{M}$, and $\left[\mathrm{H}_{2} \mathrm{SO}_{4}\right] \approx 0.78 \mathrm{M}$.

Perturbations in successive cycles may result in repeated switching of synchronization, which often occurs at different phase positions. This results in drifting of the phase perturbations into either the excitatory or the inhibitory region.

\subsection{Numerical Calculation Results}

We use a two-channel modified ZBKE model in Eq. (3.4) to carry out numerical simulations to compare with the observed experimental behavior. The parameter $\alpha_{0}$ is introduced to specify the weights of the excitatory and the inhibitory channels in the photosensitive Rucatalyzed BZ reaction. The system is purely excitatory for $\alpha_{0}=1.00$ and purely inhibitory for $\alpha_{0}=0.00$. 
(a)

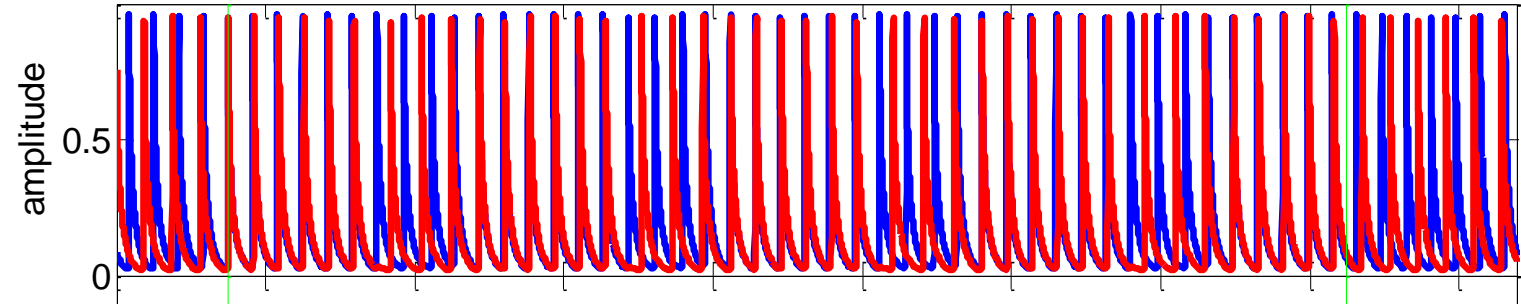

(b)

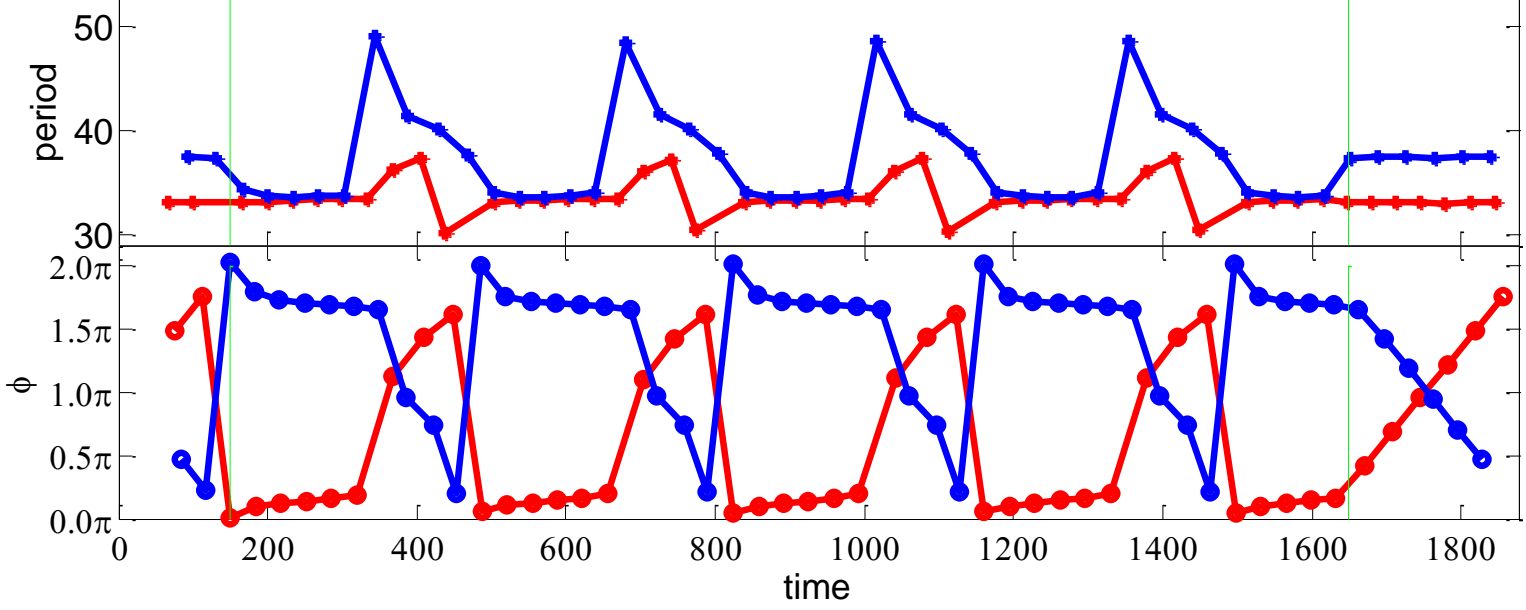

Figure 4.12: A pair of coupled oscillators displaying switching synchronization: (a) time series, (b) calculated periods, and (c) perturbation phases. Coupling is applied between the green lines. See section 3.3.1 for the modified ZBKE model with general parameters, additional specific parameters are: $\alpha_{0}=0.45, \mathrm{q}_{1}=0.97284, \mathrm{q}_{2}=0.74387$, and $\mathrm{k}=2.53 \times 10^{-3}$.

Panel (c) shows the phases at which the oscillators receive perturbations during the coupling interaction. The effect of the perturbations on the oscillator periods and the oscillation time series is shown in panels (b) and (a), respectively.

We investigate the periodicity of the switching pattern by plotting the periods as a function of time. Plotting consecutive periods allows distinguishing between periodic and aperiodic switching patterns. 


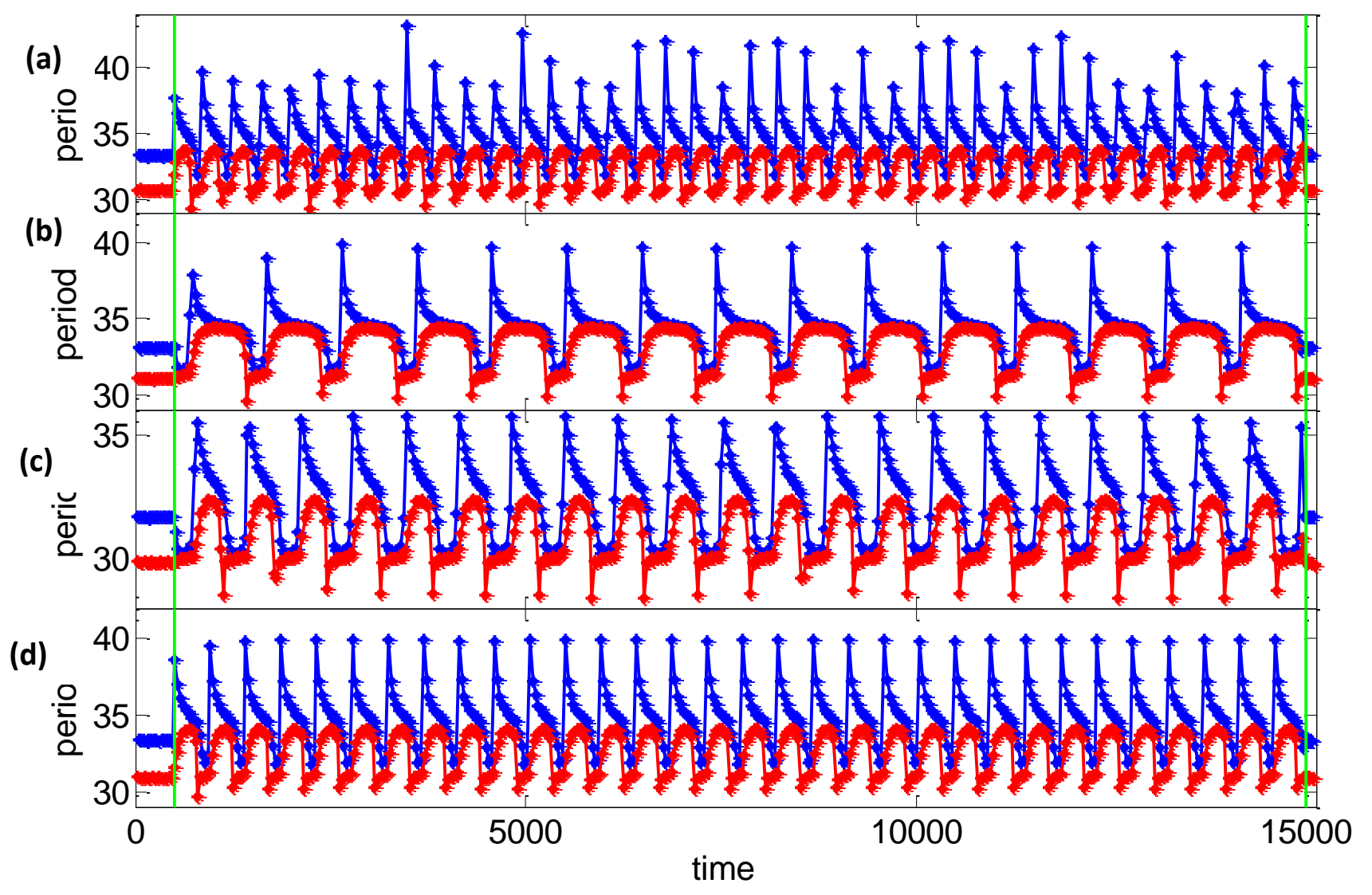

Figure 4.13: Switching synchronization patterns. Coupling is applied between the green lines. See section 3.3.1 for the modified ZBKE model with general parameters, additional specific parameters are: $\alpha_{0}=0.45$, (a) $\mathrm{q}_{1}=0.6673, \mathrm{q}_{2}=0.5426\left(\mathrm{~T}_{1}=33.1, \mathrm{~T}_{2}=30.4\right.$ ), (b) $\mathrm{q}_{1}=0.6528, \mathrm{q}_{2}=0.5598$ ( $\mathrm{T}_{1}=32.8, \mathrm{~T}_{2}=30.8$ ), (c) $\mathrm{q}_{1}=0.5875, \mathrm{q}_{2}=0.5026\left(\mathrm{~T}_{1}=31.4, \mathrm{~T}_{2}=29.6\right)$, and (d) $\mathrm{q}_{1}=0.6673$, $\mathrm{q}_{2}=0.5526\left(\mathrm{~T}_{1}=33.1, \mathrm{~T}_{2}=30.7\right)$. 


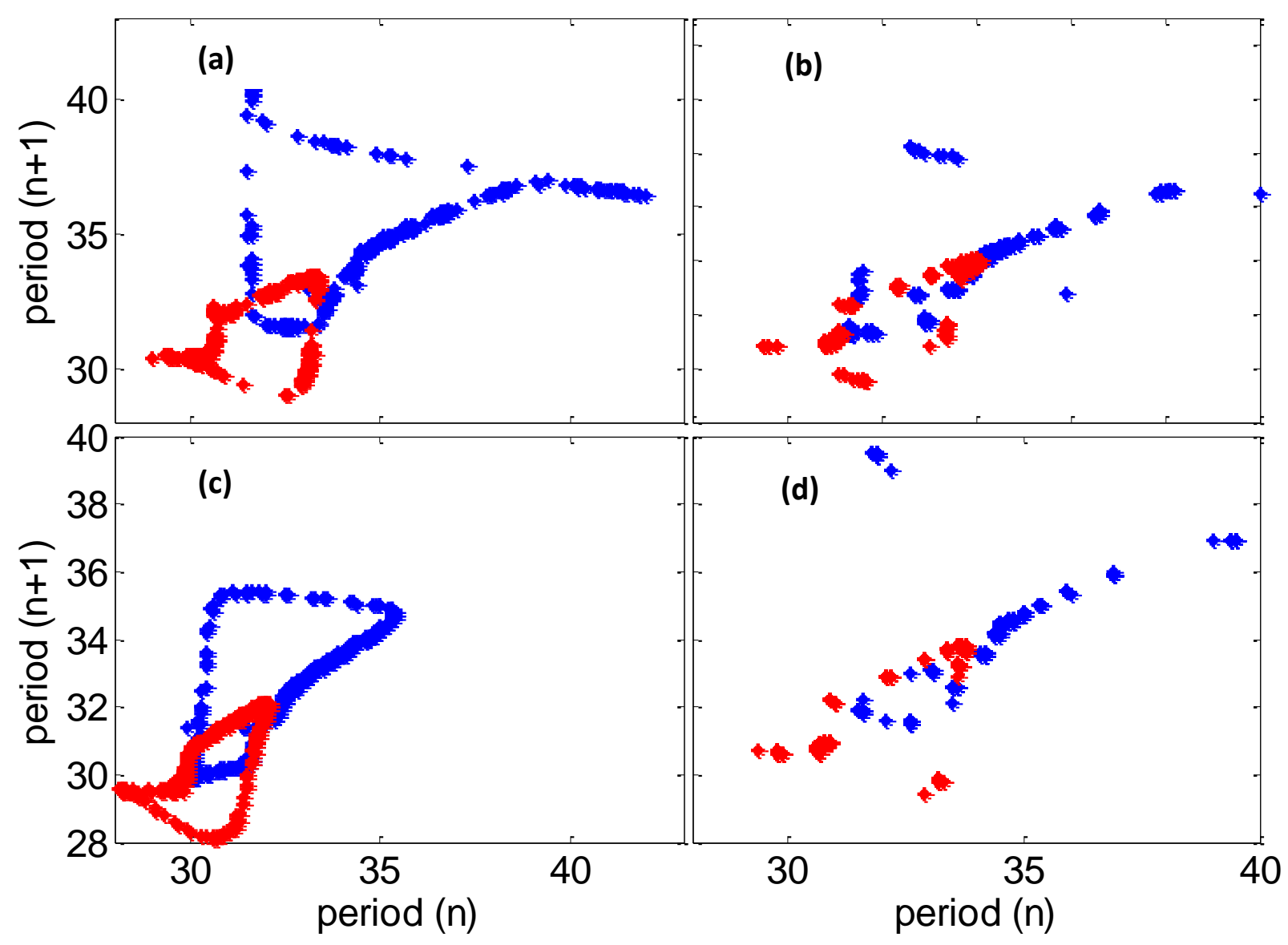

Figure 4.14: Period $(n+1)$ as a function of period $(n)$ for the switching synchronized patterns. The parameters for panels in Figure 4.14 correspond to the parameters for panels in Figure 4.13.

The plotted points follow a cycle, with panels (b) and (d) showing periodic jumps defining each cycle. The periodic pattern for (b) has 28-29 points, and (d) has 13-14 points. The aperiodic patterns for (a) and (c) have aperiodic jumps for each cycle. The plots below show the PRC and PTC. 

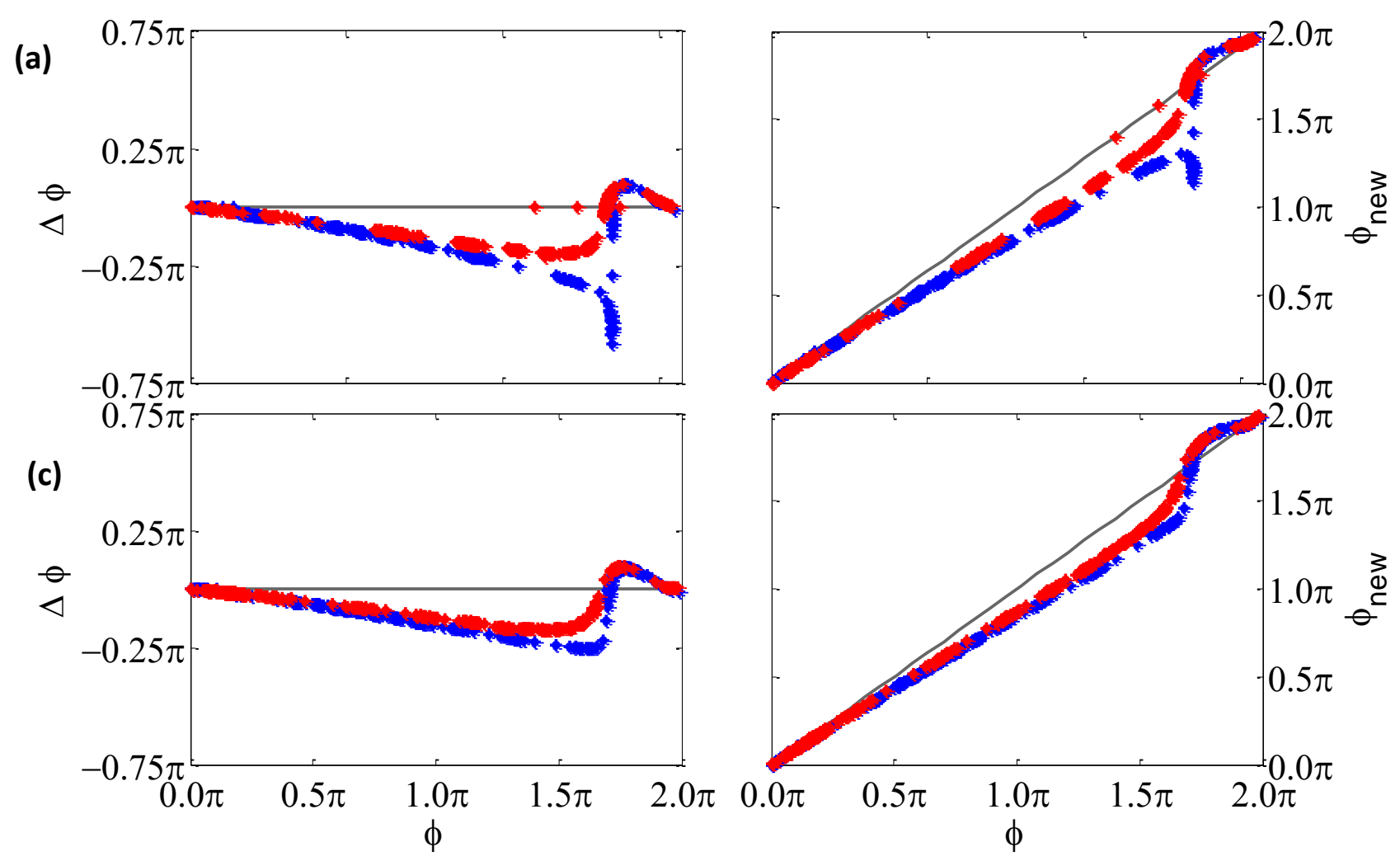

Figure 4.15: Iterative phase map (PRC and PTC). The perturbation phase of the light pulse from the coupled oscillators' interaction. The PRC shows shifting up or down according to whether the oscillators are being phase advanced or phase delayed. The parameters for panels in Figure 4.15 correspond to the parameters for panels in Figure 4.13.

Using a continuation method in numerical simulations, we carried out further calculations to investigate the effects of parameters such as the period heterogeneity, the degree of the inhibitory and the excitatory regimes, and coupling strength on the synchronization patterns. A phase diagram showing the qualitative dynamics of the system at different values of the parameters is plotted below. The phase diagram allows us to predict the presence of multistable patterns when the qualitative dynamics such as synchronization patterns changes as parameters are varied. Figure 4.16 summarizes the continuation method simulations in the form of a phase diagram, where oscillator period heterogeneity and the degree of inhibitory-excitatory regimes are varied. 


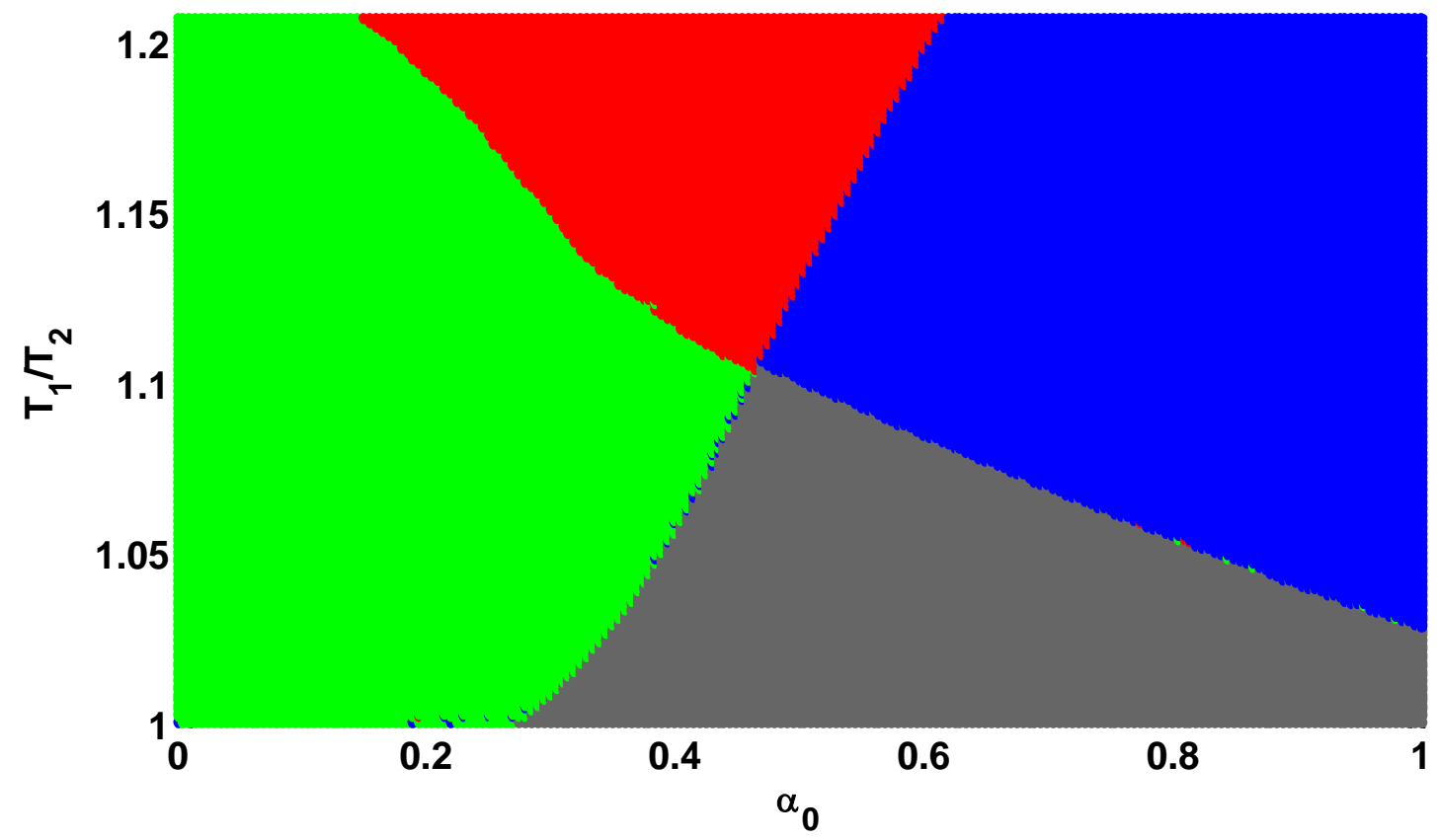

Figure 4.16: Simulations with a modified ZBKE model of heterogeneous, photosensitive BZ oscillators. The synchronization patterns with in-phase (dark blue), out-of-phase (green) synchronization patterns, bistable synchronizations (grey), and switching patterns (red) as a function of the heterogeneous oscillator period distribution and the inhibitory-excitatory regimes weight. See section 3.3.1 for the modified ZBKE model with general parameters; additional specific parameters are: $\alpha_{0}=\left[\begin{array}{ll}0.0 & 1.0\end{array}\right]$, $q_{1}=0.8580, q_{2}=\left[\begin{array}{ll}0.8543 & 0.6993\end{array}\right], k=2.53 \times 10^{-3}$.

The heterogeneity of the two oscillators and the excitatory-inhibitory regime of the BZ system play a role in influencing the resultant behaviors in two coupled BZ oscillators. Figure 4.15 above reveals four synchronization pattern regions: (i) exclusively in-phase synchronization region (dark blue), (ii) exclusively out-of-phase synchronization region (green), (iii) bistable region (grey) with coexisting in-phase and out-of-phase synchronization (depending on initial phase conditions) and (iv) switching behavior (red) with periodic and "aperiodic" patterns. Two curves separate these four regions. The in-phase region is located at the upper right of the phase diagram. The out-of-phase region is on the left of the phase diagram (green color). The bistable region is found at the lower right and is colored grey.

Simulations were carried out similarly to that described above except with varying oscillator period heterogeneity and coupling strength k, Figure 4.17. 


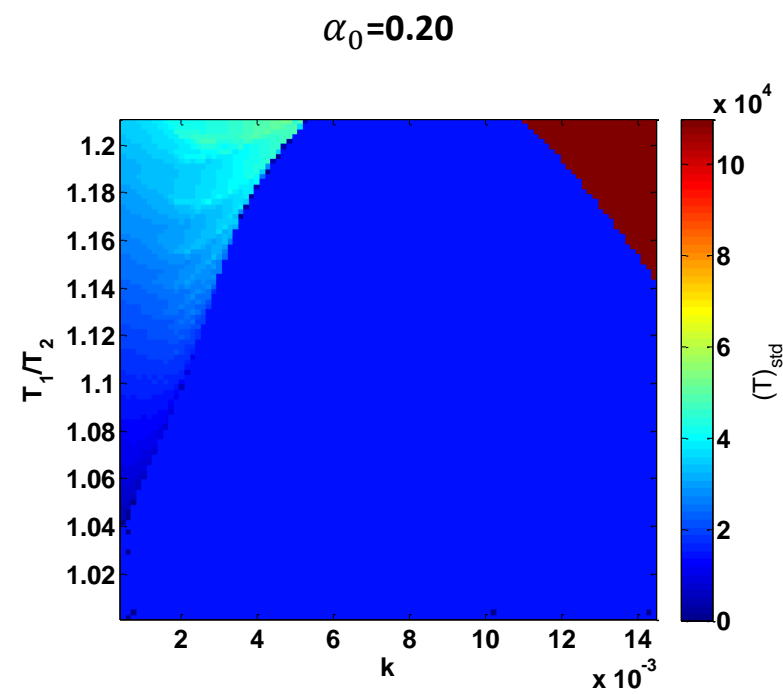

$\alpha_{0}=0.70$

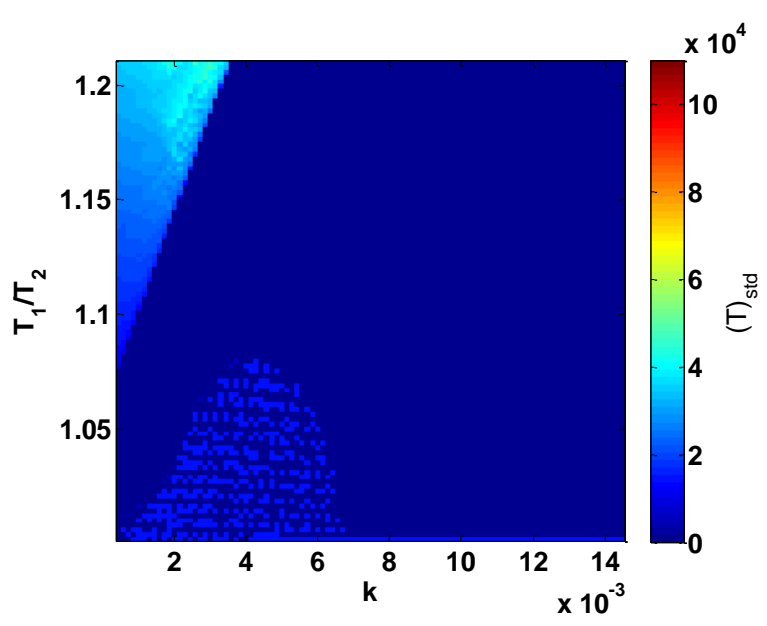

$\alpha_{0}=0.55$

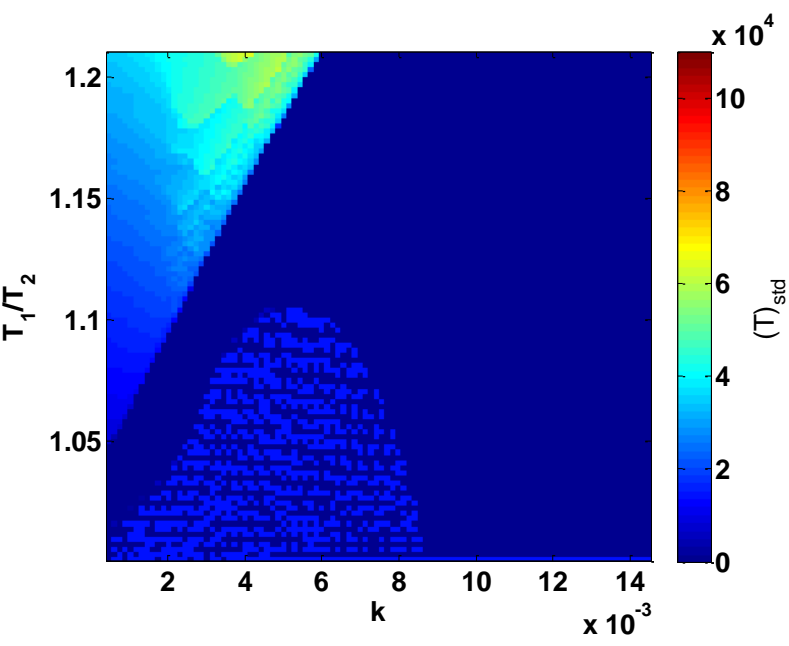

$\alpha_{0}=0.95$

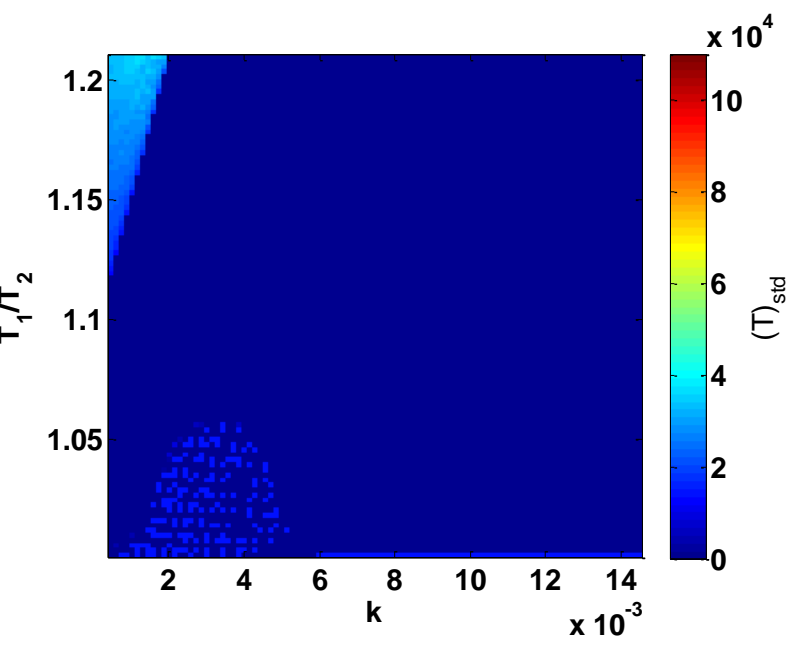

Figure 4.17: Simulations with a modified ZBKE model of heterogeneous, photosensitive BZ oscillators. The synchronization patterns with in-phase (dark blue), out-of-phase (light blue) synchronization patterns, and switching patterns (other colors) as a function of the oscillator period distribution and coupling strength, $\mathrm{k}$, in three different inhibitory-excitatory regimes weight, $\alpha_{0}$. See section 3.3 .1 for the modified ZBKE model with general parameters; additional specific parameters are: $q_{1}=0.8580$, $q_{2}=\left[\begin{array}{ll}0.8543 & 0.6993\end{array}\right]$.

Phase response curves and the associated phase transition curves are useful in understanding the dynamical behavior of coupled oscillators. Heterogeneity of the natural period of the coupled oscillators and coupling strength are also essential system parameters in characterizing the synchronization behavior. 


\subsection{Summary}

In this chapter, we examined and characterized the phase response curves and the different synchronization patterns observed when two BZ oscillators are photochemically coupled. The characterization of the BZ reaction's PRC reveals various possible degrees of different photochemical coupling channels where new dynamical behaviors can be observed. In the coupled pairs of the BZ oscillators, different regions of synchronization patterns are observed as we vary a combination of parameters such as heterogeneity, weight of inhibitory-excitatory regimes and coupling strength (shown in Figures 4.15 and 4.16). This study highlighted the importance of the PRC in providing insights into oscillator entrainment mechanisms and the effect of varying intrinsic parameters on dynamical behaviors of the coupled oscillators. We have presented experimental findings followed by numerical simulations using the modified ZBKE model. We examined the dynamics of the coupled oscillators and were able to categorize the behavior as phase-locked synchronization and switching patterns. We found both periodic and aperiodic behavior in the switching patterns.

Using numerical simulations, we investigated the effects of parameters such as period heterogeneity, degree of inhibitory-excitatory regimes, and coupling strength on the synchronization patterns observed in pairs of coupled BZ oscillators. We performed continuation method calculations to map out the regions of the dynamical behaviors observed in a phase diagram in which oscillator period heterogeneity and the degree of inhibitoryexcitatory regimes (or coupling strength) are varied. 


\section{References}

[1] P. Uhlhaas, G. Pipa, B. Lima, L. Melloni, S. Neuenschwander, D. Nikolic, and W. Singer, "Neural synchrony in cortical networks: history, concept and current status." Front. Integr. Neurosci. 3, 17 (2009).

[2] C. C. Canavier and S. Achuthan, "Pulse coupled oscillators and the phase resetting curve." Math. Biosci. 226, 77-96 (2010).

[3] K. M. Hannay, V. Booth, and D. B. Forger, "Collective phase response curves for heterogeneous coupled oscillators." Phys. Rev. E 92, 022923 (2015).

[4] J. A. Goldberg, C. A. Deister, and C. J. Wilson, "Response properties and synchronization of rhythmically firing dendritic neurons." J. Neurophysiol, 97, 208-219 (2007).

[5] Y. Tsubo, Y. Isomura, and T. Fukai, "Neural dynamics and information representation in microcircuits of motor cortex." Frontiers Syst. Neurosci. 7, 85 (2013).

[6] C. J. Wilson, B. Beverlin II, and T. Netoff, "Chaotic desynchronization as the therapeutic mechanism of deep brain stimulation." Frontiers Syst. Neurosci. 5, 50 (2011).

[7] S. Kádár, T. Amemiya, and K. Showalter, "Reaction mechanism for light sensitivity of the Ru(bpy) -catalyzed Belousov-Zhabotinsky reaction." J. Phys. Chem. A 101, 8200-8206 (1997).

[8] L. Treindl, D. Knudsen, T. Nakamura, T. Matsumura-Inoue, K. B. Jorgensen, and P. Ruoff, "The light-perturbed Ru-catalyzed Belousov-Zhabotinsky reaction: evidence for photochemically produced bromous acid and bromide ions by phase response analysis." J. Phys. Chem. A 104, 10783-10788 (2000).

[9] A. I. Lavrova and V. K. Vanag, "Two pulse-coupled non-identical, frequency-different BZ oscillators with time delay." Phys. Chem. Chem. Phys. 16, 6764-6772 (2014).

[10] V. Horvath, D. J. Kutner, J. T. Chavis III, and I. R. Epstein, "Pulse-coupled BZ oscillators with unequal coupling strengths." Phys. Chem. Chem. Phys. 17, 4664-4676 (2015).

[11] T. Sekiguchi, Y. Mori, N. Okazaki, and I. Hanazaki "Photoinduction and photoinhibition of chemical oscillations in the tris(2,2'-bipyridine) ruthenium (II)-catalyzed minimal bromate oscillator." Chem. Phys. Lett. 219, 81-85 (1994).

[12] I. Hanazaki, Y. Mori, T. Sekiguchi, and G. Rabai, "Photo-response of chemical oscillators." Phys. D 84, 228-237 (1995). 
[13] V. Gáspár, G. Bazas, and M. T. Beck, "The influence of visible light on the BelousovZhabotinskii oscillating reactions applying different catalysts." Z. Phys. Chem. (Leipzig) 264, 4348 (1983).

[14] S. Kádár, T. Amemiya, and K. Showalter, "Reaction mechanism for light sensitivity of the $\mathrm{Ru}(\mathrm{bpy})_{3}{ }^{2+}$-catalyzed Belousov-Zhabotinsky reaction. " J. Phys. Chem. A 101, 8200-8206 (1997).

[15] J. Totz, R. Snari, D. Yengi, M. R. Tinsley, H. Engel, and K. Showalter, "Phase-lag synchronization in networks of coupled chemical oscillators.", Phys. Rev. E 92, 022819 (2015).

[16] M. R. Tinsley, S. Nkomo, and K. Showalter, "Chimera and phase-cluster states in populations of coupled chemical oscillators." Nat. Phys 8, 662-665 (2012).

[17] R. Snari, M. R. Tinsley, D. Wilson, S. Faramarzi, T. I. Netoff, J. Moehlis, and K. Showalter, "Desynchronization of stochastically synchronized chemical oscillators." Chaos 25, 123116 (2015).

[18] T. Chen, M. R. Tinsley, E. Ott, and K. Showalter, "Echo behavior in large populations of chemical oscillators." Phys. Rev. X 6, 041054 (2016).

[19] A. M. Zhabotinsky, F. Buchholtz, A. B. Kiyatkin, and I. R. Epstein, "Oscillations and waves in metal-ion-catalyzed bromate oscillating reactions in highly oxidized states." J. Phys. Chem. 97, 7578 (1993). 


\section{Chapter Five}

\section{Collective Behavior of Self-Propelled Particles}

Micro-swimmers such as bacteria exhibit various types of collective motion. The dynamical behavior of the individual synthetic micro-swimmers, such as platinum-silica particles in hydrogen peroxide solutions, has been experimentally studied. However, there are not many experimental studies focused on the collective motion of agglomerated microswimmers. In this chapter, we report on experiments carried out with silica particles that were asymmetrically coated with Pt and immersed in hydrogen peroxide solutions, which is similar to the system studied by Howse et al. [4, 8] and Ke et al. [2]. However, in this study, we report on the effect of the agglomerated particles' orientation on the resultant motion pattern. The mean square displacement, step length distribution, and mean squared angular displacement are used to quantify the resultant motion dynamics.

Understanding the influence of orientation of particles in agglomerates on the motion pattern is important in exploiting the agglomerate micro-swimmers. We report experimental observations of the agglomerate motion and carry out numerical simulations of the agglomerates under different orientations of the particles. We find that motion patterns such as linear or circular trajectories emerge in the agglomerates depending on the particle orientation. 


\subsection{Introduction}

The potential industrial and medical applications of micro-swimmers have been advanced in studies of self-propelled particles in recent decades. Self-propelled particles display directed motion by harvesting energy from the surroundings. Biological systems that exhibit selfpropulsion include bacteria, algae, and biomotors, which have inspired the fabrication of nonbiological self-propelled particles such as $\mathrm{Pt}_{-} \mathrm{SiO}_{2}$ and $\mathrm{Pt}-\mathrm{Au}$ particles in solutions of hydrogen peroxide [14, 28]. However, with decreasing micro-swimmer length scale, viscous forces dominate over inertial forces. Since conventional swimming methods become ineffective at low Reynolds number, new self-propulsion mechanisms must be devised for the motion of nonbiological micro-swimmers. The microscale nonbiological self-propelled particles must overcome the effects of Brownian motion and viscosity. Researchers have carried out theoretical and experimental studies on the motion of self-propelled particles, such as spherical $\mathrm{Pt}_{-} \mathrm{SiO}_{2}$ particles in hydrogen peroxide solution $[3,4,13,25,28]$ and iridium-silica particles in $\mathrm{N}_{2} \mathrm{H}_{4}$ solution [25]. Because these particles are half-coated with a metal, they are referred to as Janus particles, named after the two-faced Roman god, Janus [9]. During de Genne's Nobel Prize lecture [9], he used the concept of "Janus grains" for the first time to describe particles with two equal surface areas with different chemical properties, such as polar and nonpolar, hydrophilic and hydrophobic. These particles can be made with different sizes and shapes. Janus particles are characterized by different chemical compositions of the two halves of the particle. The different chemical properties play a vital role in directing the motion of the selfpropelled particles.

Microorganisms, such as bacteria, direct their motion using flagella or cilia in the fluid medium [12, 14]. Kinesin, a biomotor, uses ATP hydrolysis for motion along a microtubule [24]. For synthetic micro-swimmers such as Janus particles, the motion is due to the chemical energy obtained from the surrounding solution. The motion of some Janus particles arises from chemical gradients in a process called diffusiophoresis $[2,4,8,25]$. Other chemical reactions result in propulsion mechanisms such as interfacial gradients and electrophoresis. In our experimental studies, we used platinum coated silica particles dispersed in a solution of hydrogen peroxide. The directed motion of these particles depends on the asymmetry of the 
concentration gradient created by the reaction. Agglomerates of the self-propelled particles tend to form on their dispersion in a solution of hydrogen peroxide due to volume exclusion. The dynamics of the agglomerates in a solution of hydrogen peroxide is less well understood than the dynamics of single particles. We experimentally studied the motion of self-propelled agglomerates of $\mathrm{Pt}_{-} \mathrm{SiO}_{2}$ particles in a solution of hydrogen peroxide. The investigation focuses on the influence that the orientation of the Janus particles in trimer agglomerates has on the resultant motion. We observe both linear and circular trajectories, which depend on the orientations of the particles. Using the orientations and the experimental data, we used modified Langevin equations [32] to simulate two-dimensional trajectories of the agglomerates. The complex motions are explained with the dynamic quantities extracted from the trajectory analysis of the agglomerates in a two-dimensional plane.

\subsection{Background}

In this section, we will review the challenges in designing self-propelled particles successfully, notably the effect of Brownian motion and viscous forces. We will also discuss selected experimental studies on the dynamic behavior of individual $\mathrm{Pt}_{-} \mathrm{SiO}_{2}$ particles suspended in hydrogen peroxide solutions.

Brownian motion results from random collisions between microscopic particles and the molecules of the surrounding medium $[10,20]$. The erratic motion of particles due to random collisions is named after the British botanist, Robert Brown, who discovered Brownian motion in 1827 while studying pollen grains suspended in water using a microscope. He observed that the microscopic particles were in random motion. In 1905, Albert Einstein [31, 32] explained Brownian motion in terms of random forces moving particles backward and forward in random motion. Hence, the diffusion of passive microscopic particles surrounded by a fluid medium is a result of random thermal forces acting on the microscopic particles $[18,20]$. The StokesEinstein equation gives the spatial diffusion coefficient of a particle undergoing Brownian motion:

$$
D_{t}=\frac{k_{B} T}{6 \pi \eta R}
$$


where $k_{B} T$ is the thermal energy, $\eta$ is the viscosity of the solution, and $\mathrm{R}$ is the radius of the particle. The particle also has a rotational diffusion coefficient, given by

$$
D_{r}=\frac{k_{B} T}{8 \pi \eta R^{3}},
$$

with the characteristic rotational time scale given by $\tau_{R}=\frac{8 \pi \eta R^{3}}{k_{B} T}=\frac{1}{D_{r}}$.

Theoretical investigations of Brownian motion have shown that the mean square displacement (MSD) of a Brownian particle in two-dimensions is given by

$$
<[r(\Delta t)-r(t=0)]^{2}>=\left\langle\Delta r^{2}(\Delta t)>=4 D_{t} \Delta t\right.
$$

where $r(\Delta t)$ denotes the position, $\Delta t$ the time step, and $D_{t}$ the spatial diffusion coefficient, which is the mean displacement of one Brownian particle [20]. Thus, the spatial diffusion coefficient, $D_{t}$, can be determined using the MSD and describes the strength of the stochastic force $[10,20]$.

\subsubsection{Solving the Challenges: Overcoming Brownian Motion in Microscale Particles}

A microorganism's lack of sufficient inertial force to overcome viscous resistance and the random forces due to Brownian motion make the generation of constant directed motion difficult. Therefore, micro-swimmers must possess suitable steering mechanisms to maintain orientation or use an external bias to support their motion [4]. For example, the bacterium E. coli uses rotating or wave propagating flagella to direct its motion, as depicted in Figure 5.1 [8, 14].

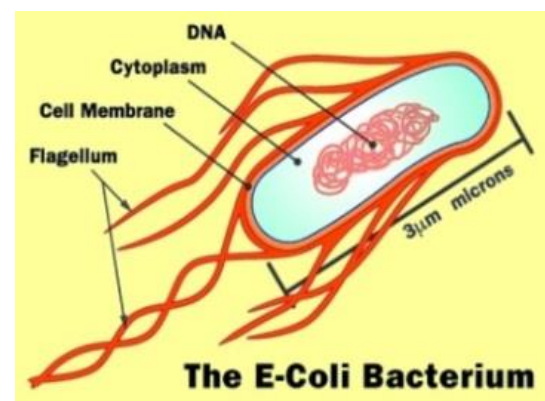

Figure 5.1: E. coli Bacterium. From Marshall Brain, HowStuffWorks.com. 
The motion of most synthetic micro-swimmers is driven by external power sources or fields, such as magnetic and electric fields [13]. Although theoretical models have been proposed to provide alternative methods for overcoming viscous resistance and the random forces due to Brownian motion, few of these models are successful without using external fields [13, 14]. Most synthetic microscale self-propelled particles using external fields are ineffective due to the lack of suitable power sources as well as the complexity of the environment. This problem calls for an alternative method that is effective and sustainable to direct particle motion autonomously.

\subsubsection{Reaction-Driven Propulsion}

The motion of micro-swimmers can be directed using an external force, but better alternative methods can be explored. The alternative method should be able to direct microswimming transporters autonomously without requiring an external driving force $[3,8]$. For particles to direct their motion, they need to generate their local fields, such as electrostatic potential, temperature, or concentration gradients in otherwise homogeneous media [8]. Most biological systems use the hydrolysis of adenosine triphosphate (ATP) to adenosine diphosphate (ADP) as a source of chemical energy to power biological motors [16-17]. The use of chemical reactions to power the motion of nonbiological micro-swimmers provides an attractive mechanism for propulsion. Possible propulsion mechanisms powered by chemical reactions include diffusiophoresis, electrophoresis, and interfacial tension gradients.

\subsubsection{Propulsion by Broken Symmetry}

Myosin and kinesin are examples of biological motors that depend on ATP hydrolysis and structural asymmetry of their filaments to direct movement [16-17]. Therefore, to successfully design self-motile devices, one must combine the concepts of autonomous chemical reaction and asymmetry. In 2005, Golestainian et al. [3] modeled a self-propelling spherical particle of radius $R$, as shown in Figure 5.2, driven by a chemical reaction on its surface. 


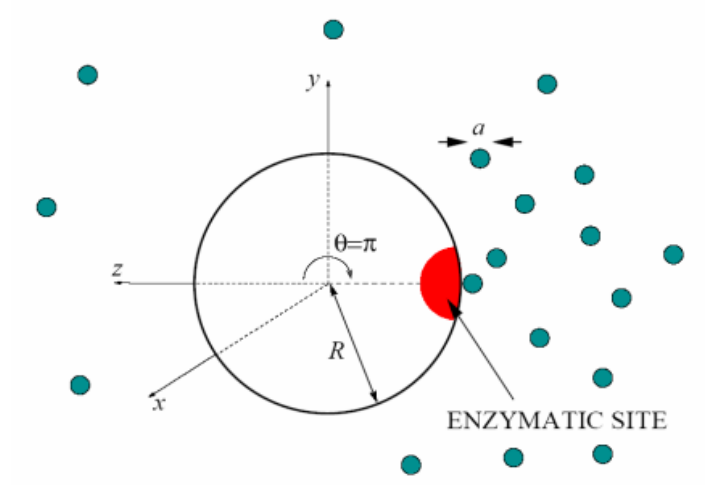

Figure 5.2: A schematic representation of a spherical particle of radius $R$ with the enzymatic/catalytic site on the surface. Figure is from Ref. [3].

If the particle is propelled by a driving force generated by a chemical reaction on its surface, the instantaneous direction of motion is parallel to the driving force. The driving force accounts for the self-propulsion, and the Janus particle receives a continuous push from the catalytic (enzymatic) site. The continuous push gives rise to directed motion with a constant velocity, $\mathrm{V}$. Using quantities from the driven particle such as the velocity, $\mathrm{V}$, and accounting for the influence of translational and rotational fluctuations, we can use Langevin equations [33] to describe the particles propulsion in a two-dimensional plane $(x-y)$, as shown below $[23,26,27]$ :

$$
\begin{aligned}
& \frac{d x(t)}{d t}=V \cos (\theta(t))+\sqrt{2 D_{t}} \xi_{1}(t), \\
& \frac{d y(t)}{d t}=V \sin (\theta(t))+\sqrt{2 D_{t}} \xi_{2}(t), \\
& \frac{d \theta(t)}{d t}=\sqrt{2 D_{r}} \xi_{r}(t),
\end{aligned}
$$

where $(x, y)$ is the position of the particle center of mass, $\xi_{1}$ and $\xi_{2}$ are terms for translational fluctuations, $\xi_{r}$ is the term for rotational fluctuations, and $\theta$ is the angle of orientation.

Using the model illustrated in Figure 5.2, Howse et al. [4] synthesized self-propelled particles powered by the process of diffusiophoresis, which arises when the catalytic decomposition of hydrogen peroxide occurs on the platinum coated silica particle. The Langevin equations can be used to simulate the self-propulsion of the particle. 


\subsubsection{Hydrogen Peroxide Fueled Platinum-Silica Janus Particles}

Experimental studies of Janus particles dispersed in a solution of hydrogen peroxide provide insights into the dynamical properties of self-propelled particles. Platinum catalyzes the decomposition of hydrogen peroxide into oxygen and water,

$$
2 \mathrm{H}_{2} \mathrm{O}_{2} \stackrel{\mathrm{Pt}}{\rightarrow} \mathrm{O}_{2}+2 \mathrm{H}_{2} \mathrm{O},
$$

on one-half of the spherical particle, thereby generating a concentration gradient in the interfacial region [1, 2, 4, 8, 29]. The reaction shown by Eq. (5) occurs as illustrated in Figure 5.3 , and is responsible for the propulsion of the particle. Self-propelled motion has been observed with the catalytic region at the trailing end of the sphere $[2,4,8]$.

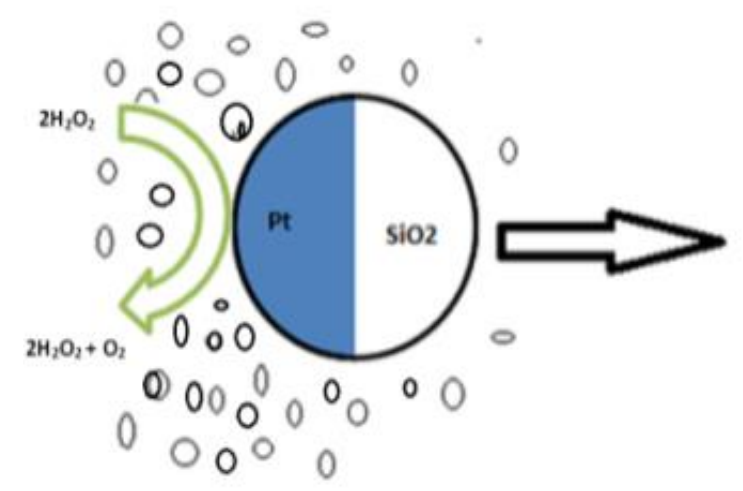

Figure 5.3: Schematic representation of Janus $\mathrm{Pt}_{-} \mathrm{SiO}_{2}$ particle undergoing diffusiophoresis.

Howse et al. $[1,4,8]$ investigated the motion of the self-propelled particles by dispersing platinum coated polystyrene spheres in a hydrogen peroxide solution. Propulsion was due to diffusiophoresis, which occurs when the particle is subjected to a concentration gradient in the solution, as illustrated in Figure 5.3. In the diffusiophoresis mechanism, the reaction of platinum with hydrogen peroxide induces a local asymmetric production of oxygen that leads to a spatial concentration gradient. The local asymmetric production of oxygen gives rise to the propulsion of the $\mathrm{Pt}_{-} \mathrm{SiO}_{2}$ particles. The propulsive motion strongly depends on the concentration of hydrogen peroxide. 


\subsection{Experimental Method}

\subsubsection{Platinum-Silica Particles Fabrication}

An aqueous suspension of silica microspheres ( $1 \mu \mathrm{m}$ diameter) purchased from Bangs Laboratories was deposited on a glass slide. The glass slide, now covered in a closely packed silica micro-sphere monolayer, is allowed to dry at room temperature overnight. After drying, the monolayer can be coated with platinum metal using electron beam vacuum evaporation, as illustrated in Figure 5.4 [1]. The microspheres were half-coated with a platinum film approximately $5 \mathrm{~nm}$ in thickness. The asymmetric particles that formed on the glass slides were immersed in deionized water and removed by sonication.

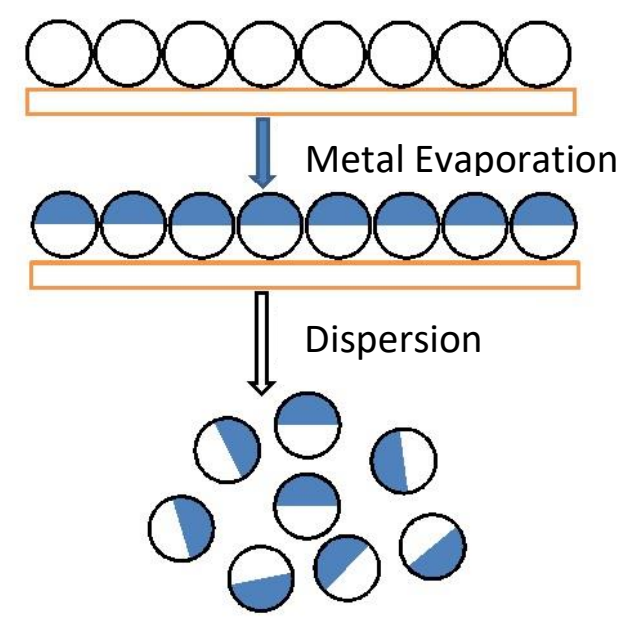

Figure 5.4: A schematic illustration of the preparation of Janus particles.

\subsubsection{Experimental Procedure}

Self-propelled particles made of silica spheres ( $1 \mu \mathrm{m}$ in diameter) and half coated with platinum were dispersed in water during sonication. The particles suspended in water were mixed with hydrogen peroxide solution to obtain a dispersion with the desired concentration. The dispersion was placed on a glass slide. The glass slide, with a thickness of $0.18 \mathrm{~mm}$, was used to position the suspended particles on an inverted microscope (Lecia, 100× objective lens with immersion oil) connected to a high-resolution color digital camera (SPOT 100), as shown in the setup in Figure 5.5. On the surface of the glass slide, two-dimensional motion is exhibited 
by the particles. A small region was selected from the field view containing particles to maximize the frame capture rate. For our instrumentation setup below, images were typically collected at 9-16 fps. The videos contained the frame-to-frame information for movement and orientation of the particles. The positions of the particles in the two dimensions with respect to time were determined frame to frame. The two-dimensional $(x-y)$ trajectories and particles' orientation from the image sequences were processed using ImageJ software for detailed analysis with MATLAB. Deionized water was used for all experiments and solution preparations. All experiments were carried out at room temperature.

\subsubsection{Instrumentation}

The experimental set-up in Figure 5.5 consists of a color digital camera (Spot Insight) to capture two-dimensional image sequences, using an inverted microscope (Leica DM IRB) with a 100x objective. The platinum-silica particles in a solution of hydrogen peroxide were dispersed on glass slides. The image sequences were collected and were stored in the computer (PC).

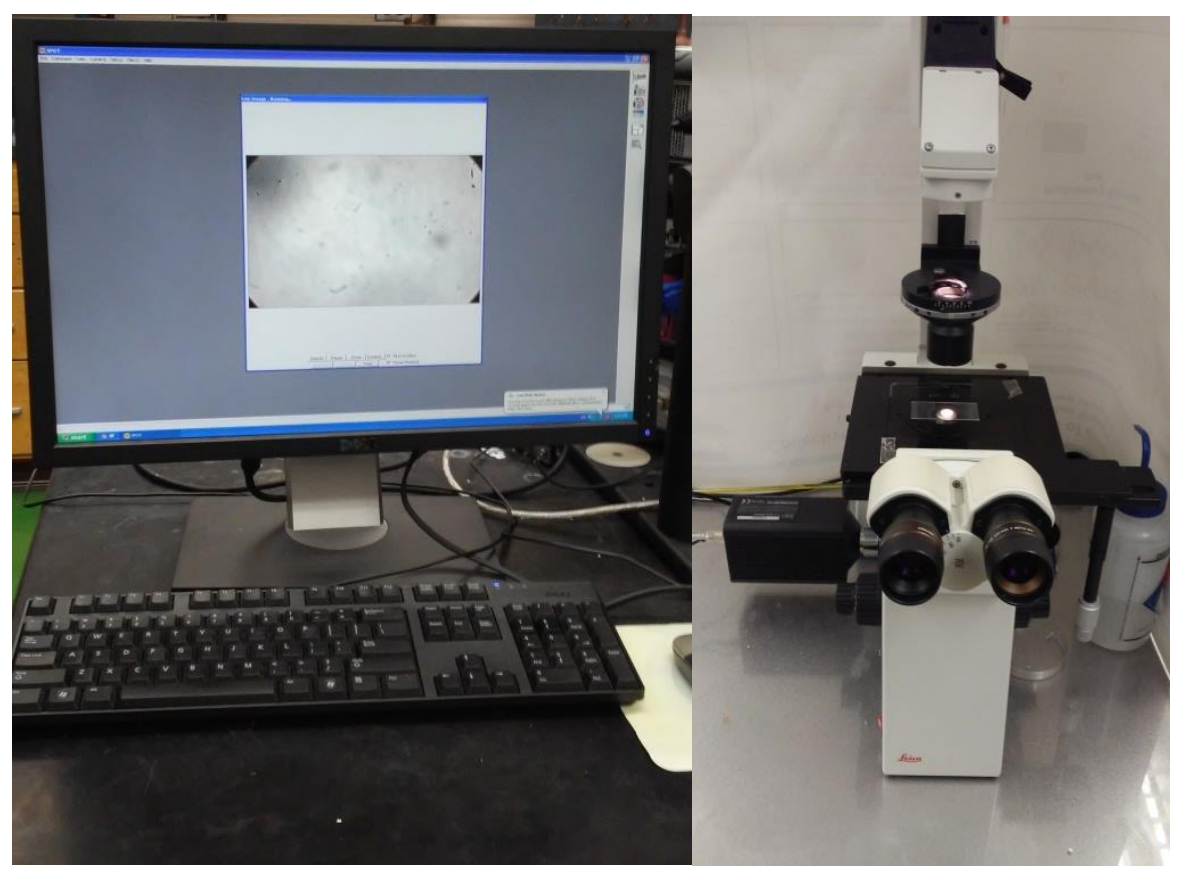

Figure 5.5: Experimental set up. 


\subsection{Analysis of the motion of self-propelled particles}

The frame-to-frame tracking and measurement of the position and orientation of the particles on sequential images at constant time intervals allow for the determination of dynamic quantities that characterize the motion. Two methods have been utilized to quantify the dynamics of self-propelled particles in previous work, the mean square displacement (MSD) and the step length distribution. Previous work on single self-propelled particles is described to illustrate how these methods quantify and extract motion information. We apply these methods to analyze experimental and numerical simulation data for agglomerates of these particles in our study.

Figure 5.6 shows a comparison of the trajectories of $\mathrm{Pt}_{-} \mathrm{SiO}_{2}$ particles with equivalent pure silica spheres serving as the control at different hydrogen peroxide concentrations $[4,8]$.

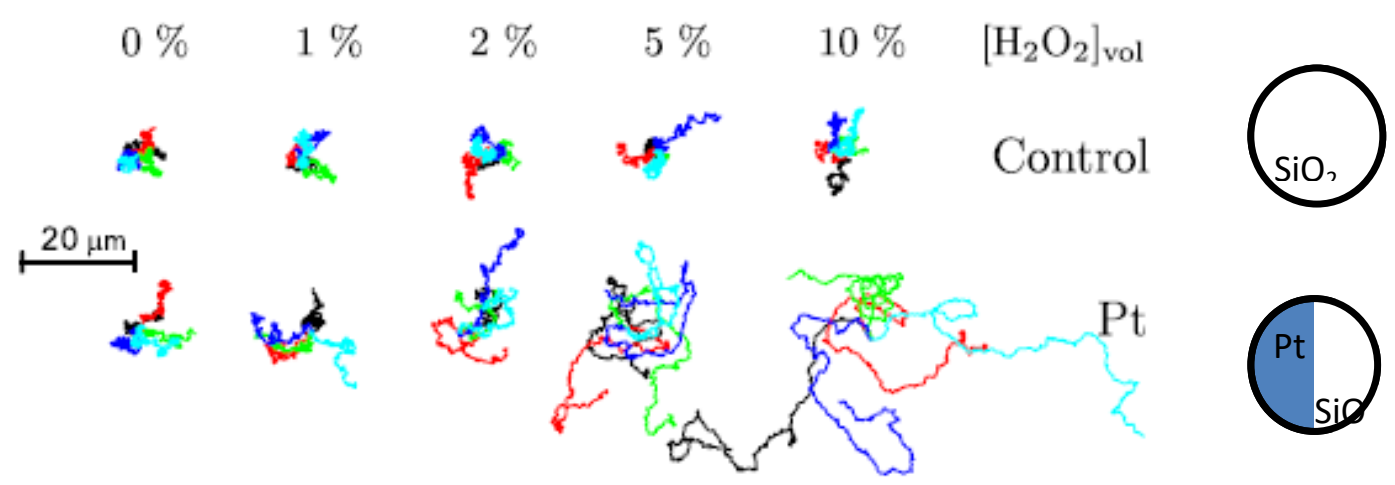

Figure 5.6: Trajectories of $\mathrm{Pt}-\mathrm{SiO}_{2}$ and control pure silica particles at different hydrogen peroxide concentrations. Figure adapted from Ref. [4].

The mean-squared displacement can characterize the dynamics of the $\mathrm{Pt}_{-} \mathrm{SiO}_{2}$ particles in different concentrations of hydrogen peroxide. In a two-dimensional space, the MSD is given by

$$
\Delta L_{(\Delta t)}^{2}=<\left(\Delta x_{(\Delta t)}^{2}+\Delta y_{(\Delta t)}^{2}\right)>
$$

where $\Delta x_{(\Delta t)}=x_{(t+\tau)}-x_{(\tau)}$, and $\Delta y_{(\Delta t)}=y_{(t+\tau)}-y_{(\tau)}$.

As the concentration of the hydrogen peroxide increases, the motion of the selfpropelled particles leads to characteristic deviations of the MSD in comparison to the ordinary Brownian motion. It is clear from Figure 5.7 that with increasing hydrogen peroxide concentration, the plots of MSD against time become increasingly exponential. The deviation of 
the MSD arises from the propulsive component of the particle motion, which increases with increasing hydrogen peroxide concentration $[4,8]$.

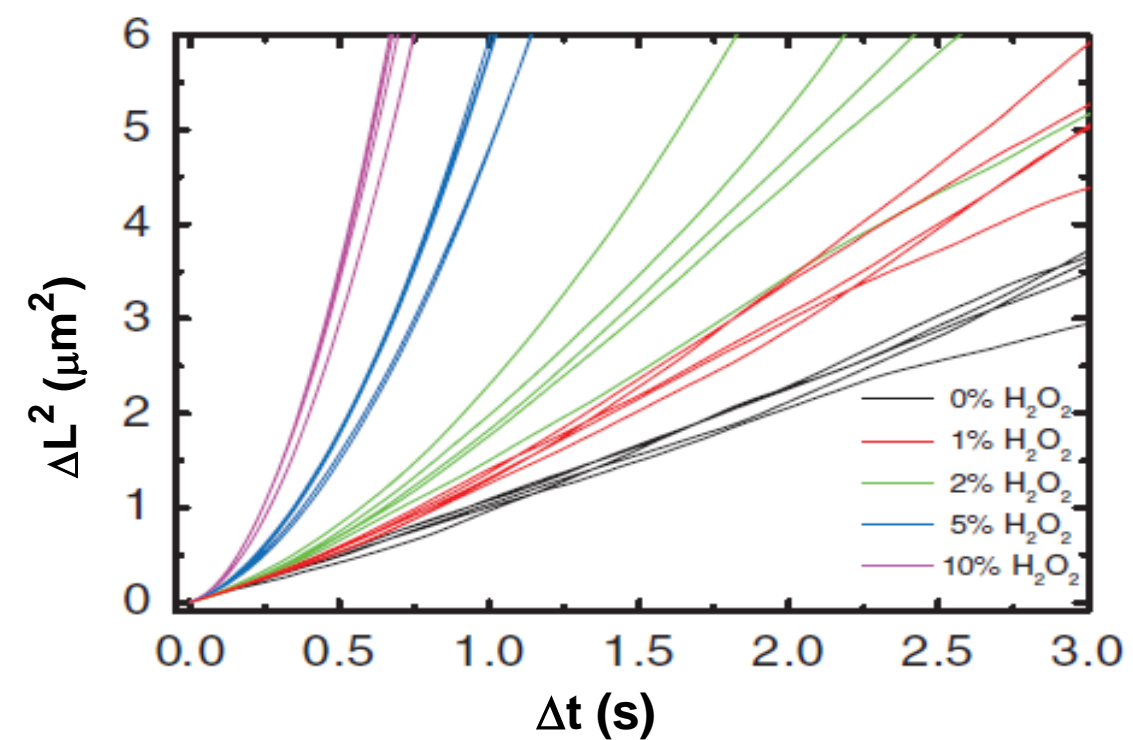

Figure 5.7: Plot of mean-square displacement $\left(\Delta \mathrm{L}^{2}\right)$ as a function of time $(\Delta t)$ of trajectories in Figure 5.6. Figure adapted from Ref. [8].

The two-dimensional MSD can be related to the dynamics of propulsion by the equation below $[4,8,27]$.

$$
\Delta L_{(\Delta t)}^{2}=4 D_{t} \Delta t+\frac{v^{2} \tau_{R}^{2}}{2}\left(\frac{2 \Delta t}{\tau_{R}}+e^{\frac{-2 \Delta t}{\tau_{R}}}-1\right),
$$

Howse et al. in their analysis of experimental data have shown that at short time steps, $\Delta t<$ $\tau_{R}$, the mean-squared displacement plot can be fitted by a quadratic equation

$$
\Delta L_{(\Delta t)}^{2}=4 D_{t} \Delta t+v^{2} \Delta t^{2},
$$

and the characteristic motion of the particle becomes Brownian-like at a long time step, $\Delta t>\tau_{R}$, which can be fitted by a linear equation

$$
\Delta L_{(\Delta t)}^{2}=4\left(D_{t}+\frac{1}{4} v^{2} \tau_{R}\right) \Delta t
$$

Alternatively, the mean square angular displacement (MSAD) can be utilized for a dominant rotational motion, to extract dynamic quantities.

The step length distribution of a particle trajectory in the $x$ and $y$ directions for a given observation time interval, $\Delta t$, can be used to quantify the dynamics of the particle motions. The frame-to-frame measurements of the particle trajectory allow us to determine the influence of 
$\Delta t$ on the apparent motion of the particles. The probability density for a one-dimensional displacement $\Delta$ is given by

$$
P(\Delta, \Delta t)=\exp ^{\left(\frac{-\Delta^{2}}{2 \sigma^{2}}\right)}
$$

where $\sigma=\sqrt{2 D_{\text {eff }} \Delta t}$.

The value of $D_{\text {eff }}$ can be determined by fitting the probability density from Eq. 10 to the step length $\Delta$ normal distribution for a varied time interval, $\Delta t$. Ke et al. have demonstrated that at a short time interval, $\Delta t<\tau_{R}$, the effective diffusion constant is given by a linear equation

$$
D_{\text {eff }}=D_{t}+\frac{1}{4} v^{2} \Delta t \text {. }
$$

The characteristic motion of the particle becomes Brownian-like at a long time interval, $\Delta t>$ $\tau_{R}$, and the horizontal fit on the graph plateau corresponds to the effective diffusion constant,

$$
D_{\text {eff }}=D_{t}+\frac{1}{4} v^{2} \tau_{R}
$$

where $v$ is the particle velocity of propulsion, $D_{t}$ is the diffusion constant for purely Brownian motion, and $\tau_{R}$ is the rotational diffusion time, which can be estimated as the initial time at which effective diffusion $D_{e f f}$ plateaus to a constant value. The effective diffusion, $D_{e f f}$, increases with increasing hydrogen peroxide concentrations. The dynamic quantities extracted by the analysis based on the step length distribution, mean square displacement (MSD), and mean square angular displacement (MSAD) can be compared for consistency.

Most previous studies have focused on the dynamical behavior of single self-propelled particles. Here, we examined experimentally and numerically the collective motion in agglomerates of asymmetric particles such as trimers and tetramers. The investigation focuses on interacting particles that are in contact, known as agglomerates. We examine how the relative orientation of Janus particles in trimers, tetramers, and higher agglomerates can influence the resultant motion. We present an analysis of self-propelled-particle agglomerate motion. The analysis allows us to quantify the motion pattern by extracting the diffusion constant, the rotational diffusion constant, rotational time, velocity and angular velocity using MSD, the step length distributions in the $x$ and $y$ directions, and MSAD. 


\subsection{Experimental Results}

At sufficiently high densities of $\mathrm{Pt}-\mathrm{SiO}_{2}$ particles in hydrogen peroxide solutions, the motions of particles are accompanied by the formation of agglomerates, as shown in Fig. 5.8. This clumping is thought to be due to volume exclusion, which results in adhesive interactions between the particles.

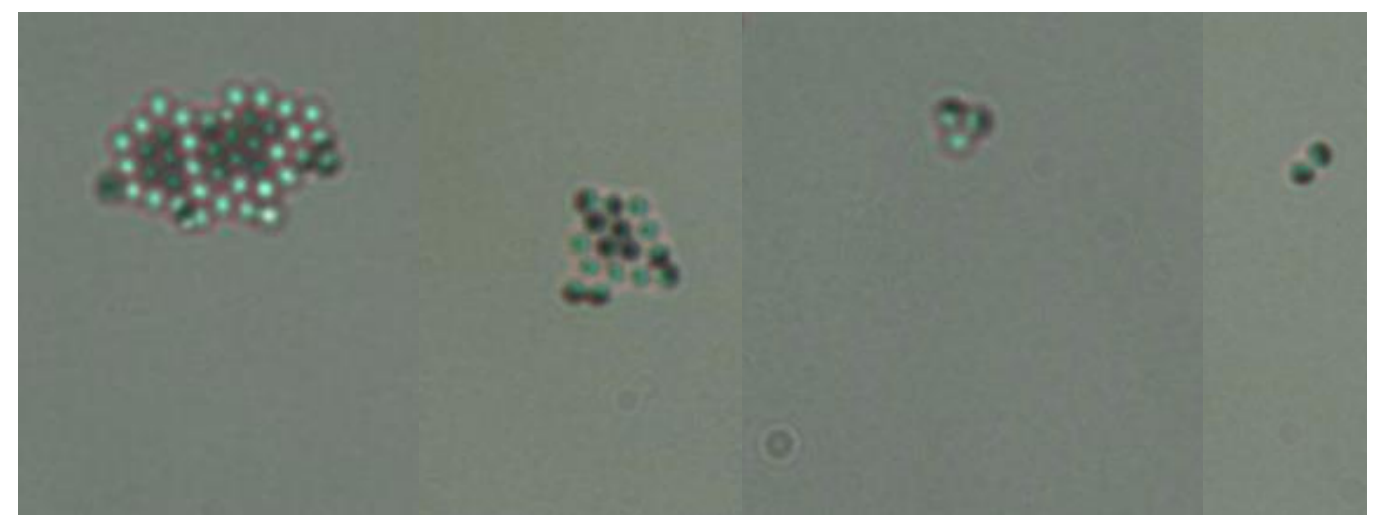

Figure 5.8: Experimental images showing $\mathrm{Pt}_{-} \mathrm{SiO}_{2}$ particles agglomerates.

Figure 5.9 shows a schematic illustration of the agglomerates formed with various orientations of the constituent particles. In the hydrogen peroxide solution, the agglomerate is propelled near the interfaces of the solution with catalytic sites due to chemical gradients generated at the individual sites. Depending on the orientation of the agglomerate, the chemical gradients developed at the multiple sites determine the motion pattern. The multiple chemical gradients, coupled with the interactions of these gradients can explain the complex motion of the agglomerates. Experimental measurements show that the agglomerates display a variety of trajectories, such as circular, rotational, spiral, and orbital, as well as linear and Brownian motion. We demonstrate here how the orientations of the $\mathrm{Pt}_{-} \mathrm{SiO}_{2}$ particles in the agglomerates, using trimers as a case study, influence the resultant motion pattern. We experimentally and numerically show the dependence of the motion patterns in the agglomerates on the particle orientations. 


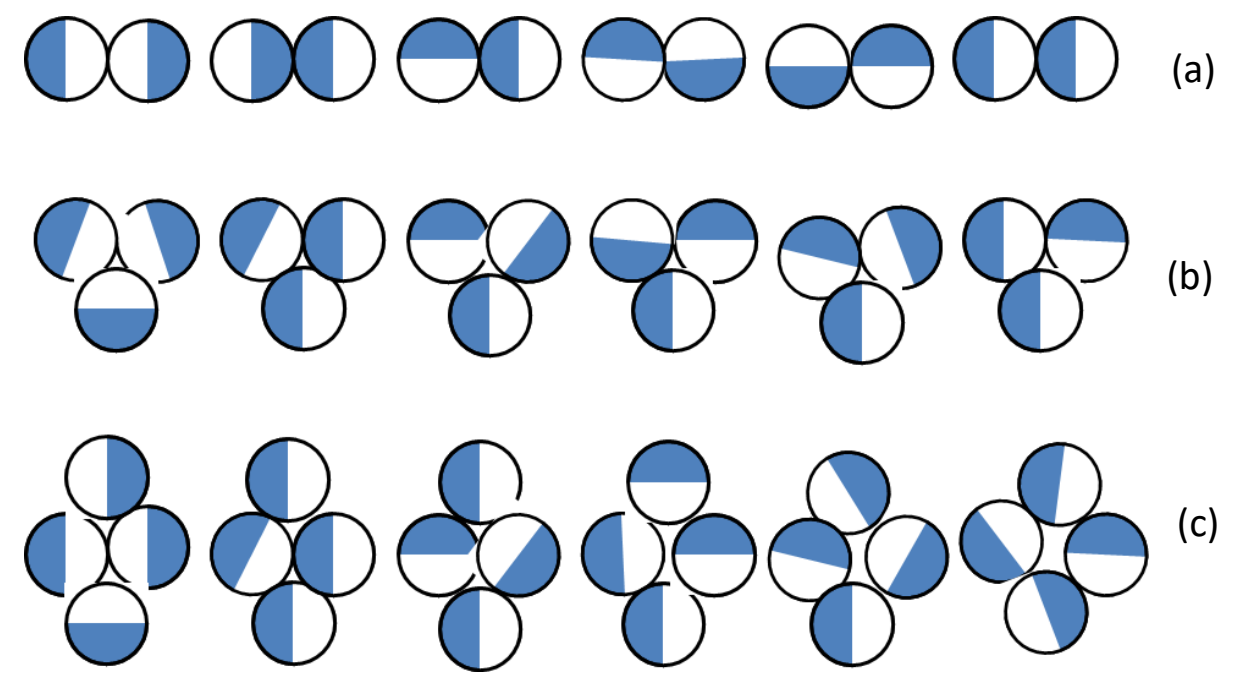

Figure 5.9: Illustration of $\mathrm{Pt}_{-} \mathrm{SiO}_{2}$ particle agglomerates, (a) dimers, (b) trimers, and (c) tetramers, showing some possible relative particle orientations. Blue and white represent platinum and silica surfaces, respectively.

The agglomeration of the self-propelled particles can be with various orientations as illustrated in Figure 5.9. We monitor the motion of these agglomerates using 2D image sequences taken by a high-resolution camera. During the analysis, we track the trajectories of the agglomerates to extract translational and rotational velocities of the motion. The pattern of motion in agglomerates of these particles can be controlled by varying the particle orientations, which modifies the relative prominence of the rotational or translational dynamics of the motion. Changing the pattern of motion by altering the orientations of the particles may allow new designs of chemically powered self-propelled particles for specialized functions.

The orientations of the self-propelled particles in agglomerate influence both the pattern and the velocities of motion. We quantify the observed resultant motions using MSD, MSAD, and the use of probability density function on the step length distribution of a selfpropelled particle in the $\mathrm{x}$ and $\mathrm{y}$ directions for varied observations time intervals. 
(a)

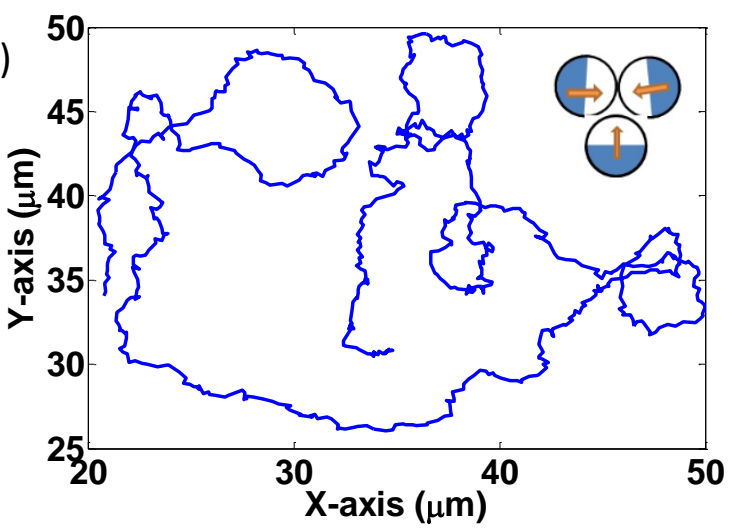

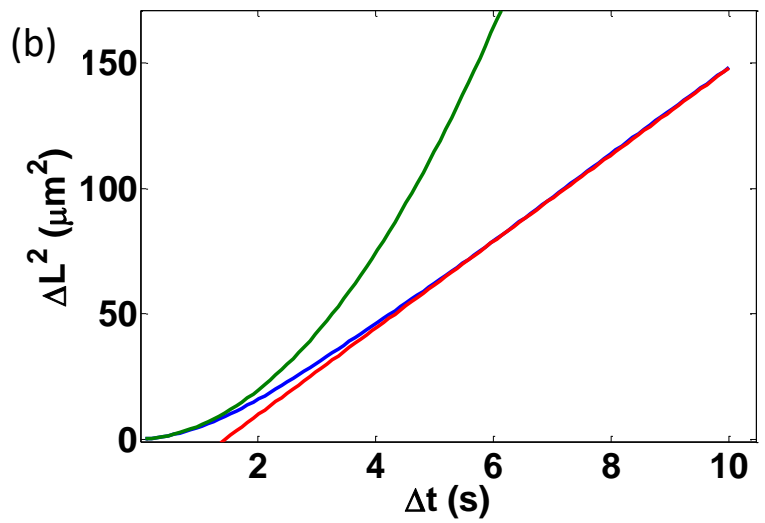

Figure 5.10: Motion of half-coated Pt-silica trimer in 5.0\% w/w hydrogen peroxide solution. The orientations of the Pt-silica particles are shown in the insert of (a). The image sequences were collected at $10.40 \mathrm{fps}$. a) The trajectory of trimer and b) mean squared displacement as a function of time steps (blue line) fitted by Eq. 7. At short times, $\Delta t<\tau_{R}$, Eq. 8 is fitted by the parabola shown by the green line, and for long times, $\Delta t>\tau_{R}$, a straight line is fitted by Eq.9 (red line). The fits yield the characteristic quantities, $v=2.10 \mu \mathrm{m} \mathrm{s}^{-1} ; D_{t}=0.221 \mu \mathrm{m}^{2} \mathrm{~s}^{-1}$, and $\tau_{R}=3.66 \mathrm{~s}$.

Using the MSD, we determine the characteristic velocity and diffusion coefficient of the particle propulsion at short times. The green curve in Figure 5.10 (b) shows a fit of the data with Eq. 8, giving $v=2.10 \mu \mathrm{ms}^{-1}$, and $D_{t}=0.221 \mu \mathrm{m}^{2} \mathrm{~s}^{-1}$. The effective diffusion, $D_{\text {eff }}$, was determined using the long time behavior of the MSD shown by the linear red fit using Eq. 9, giving $D_{\text {eff }}=D_{t}+\frac{1}{4} v^{2} \tau_{R}=4.26 \mu m^{2} s^{-1}$, and $\tau_{R}=3.66 s$.

Alternatively, we can fit the probability density function of the step length distribution of the $x$ and $y$ displacement to extract the dynamic quantities. The step length distributions are plotted for frame-to-frame 1D step lengths and normalized by dividing by the total number of steps. The orange line in Figure 5.11 (a) shows the Gaussian fit. 


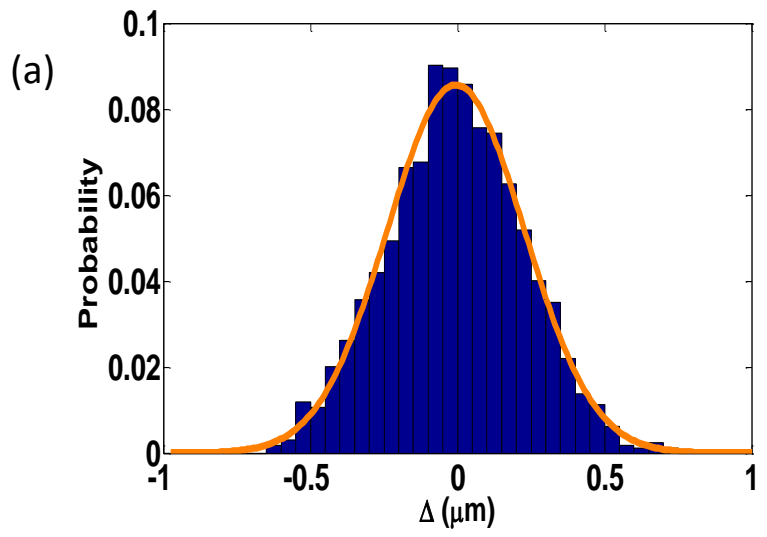

(b)

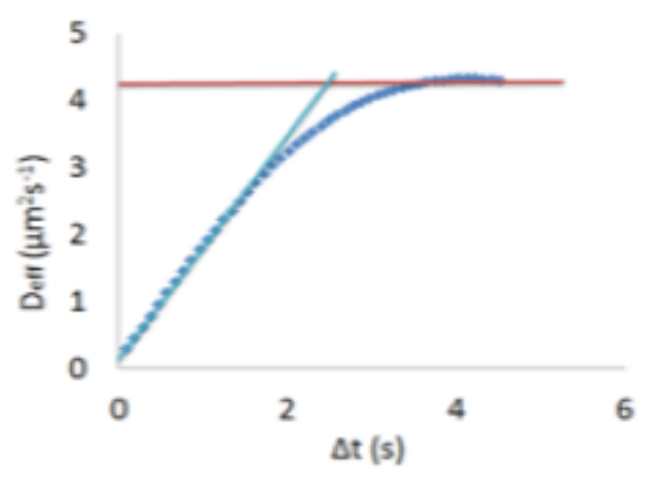

Figure 5.11: (a) Distribution of the step length $\Delta$ for trimer Pt-silica particles. (b) Effective diffusion coefficient $D_{\text {eff }}$ as a function of the observation time interval, $\Delta t$.

The dynamics quantities can be extracted using the following equations:

$$
\begin{aligned}
& \Delta t<\tau_{R}, \quad D_{e f f}=D_{t}+\frac{1}{4} v^{2} \Delta t, \text { for green line fitting. } \\
& \therefore v=2.48 \mu \mathrm{m} \mathrm{s}^{-1} ; D_{t}=0.227 \mu \mathrm{m}^{2} \mathrm{~s}^{-1} . \\
& \Delta t>\tau_{R}, \quad D_{e f f}=D_{t}+\frac{1}{4} v^{2} \tau_{R}, \text { for red line fitting. } \\
& \therefore D_{\text {eff }}=4.30 \mu \mathrm{m}^{2} \mathrm{~s}^{-1}, \tau_{R}=2.65 \mathrm{~s}, D_{r}=0.38 \mathrm{rad}^{2} \mathrm{~s}^{-1} .
\end{aligned}
$$

The values $v, D_{t}$, and $D_{\text {eff }}$ obtained from the MSD and probability density function fit for the step length distribution measurements are compared in Table 5.1,

\begin{tabular}{|l|l|l|l|l|l|}
\hline & \multicolumn{1}{|c|}{$\tau_{R}(\mathrm{~s})$} & $D_{r}\left(\mathrm{rad}^{2} \mathrm{~s}^{-1}\right)$ & $v\left(\mu \mathrm{m} \mathrm{s}^{-1}\right)$ & $D_{t}\left(\mu \mathrm{m}^{2} \mathrm{~s}^{-1}\right)$ & $D_{\text {eff }}\left(\mu \mathrm{m}^{2} \mathrm{~s}^{-1}\right)$ \\
\hline $\mathrm{MSD}$ & 3.66 & 0.27 & 2.10 & 0.22 & 4.26 \\
\hline$\Delta$ Distribution & 2.65 & 0.38 & 2.48 & 0.23 & 4.30 \\
\hline
\end{tabular}

Table 5.1: Values of $\tau_{R}, D_{r}, v, D_{t}$, and $D_{e f f}$ from MSD and step length probability density distribution $(\Delta$ Distribution) measurements.

The propelled trimer above describes linearly directed motion, and it is dominated by translational as opposed to rotational motion. The difference in the rotational diffusion coefficient, $D_{r}$, obtain by MSD and $\Delta$ Distribution might be due to a gradient field generated by the interaction of multiple catalytic sites that characterize the agglomerates. 
(a)

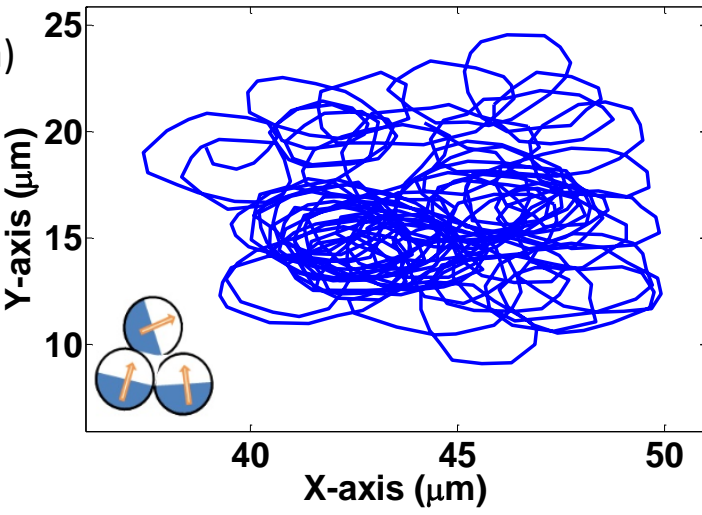

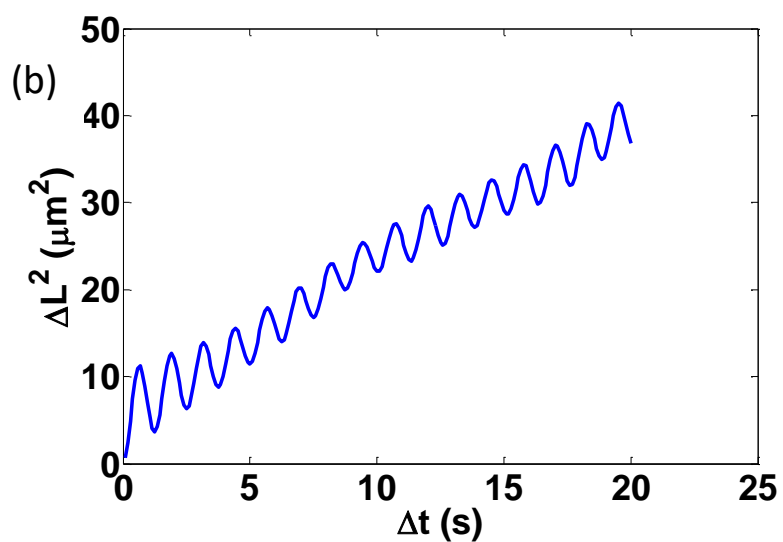

Figure 5.12: Circular trajectory and rotation motion of half-coated Pt-silica trimer in $5.0 \% \mathrm{w} / \mathrm{w}$ hydrogen peroxide solution. The orientations of the Pt-silica particles are shown in the insert at (a).The image sequences were collected at $10.40 \mathrm{fps}$. (a) The trajectory of a trimer and (b) mean square displacement.

(a)

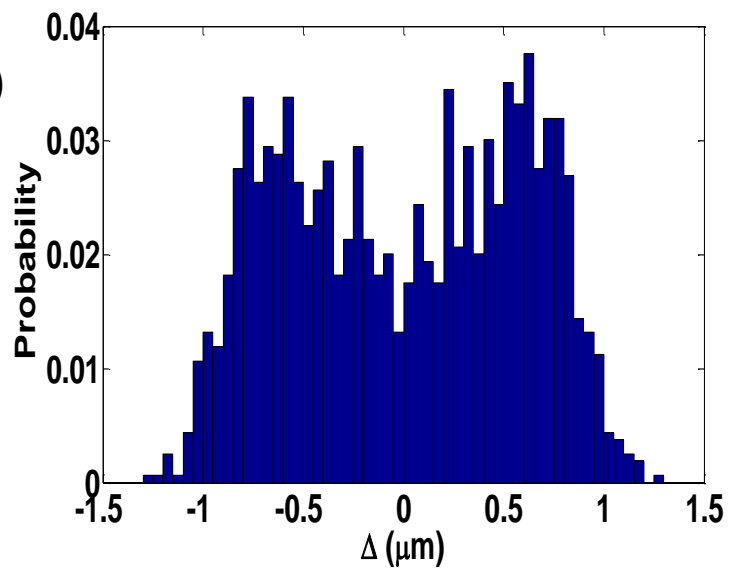

(b)

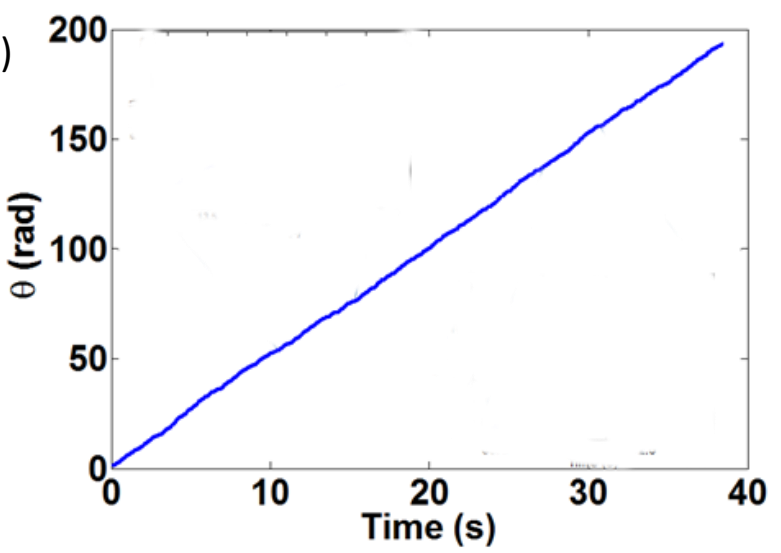

Figure 5.13: (a) Distribution of the step length $\Delta$ for trimer Pt-silica particles. (b) The time variation of $\Theta(t)$ in plots of cumulative rotational angle over time.

The time variation of $\Theta(t)$ in Figure 5.13 (b) shows that the circular motions were accompanied by monotonic rotation of the agglomerate with minor fluctuations. Oscillatory motions of the agglomerate were observed, as shown by MSD plot on Figure 5.12 (b). We can utilize an analytical fit, Eq. (13) [8], and a MSAD fit, Eq. (14) [30], to quantify the dynamics of the trajectory of motion shown in Figure 5.12 (a).

$$
\begin{aligned}
& \Delta L^{2}=4 D_{t} \Delta t+\frac{2 v^{2} D_{r} \Delta t}{D_{r}^{2}+\omega^{2}}+\frac{2 v^{2}\left(\omega^{2}-D_{r}^{2}\right)}{\left(D_{r}^{2}+\omega^{2}\right)^{2}}+\frac{2 v^{2} e^{-D_{r} \Delta t}}{\left(D_{r}^{2}+\omega^{2}\right)^{2}}\left[\left(D_{r}^{2}-\omega^{2}\right) \cos (\omega \Delta t)-2 \omega D_{r} \sin (\omega \Delta t)\right] \\
& \Delta \Theta^{2}=2 D_{r} \Delta t+\omega^{2} \Delta t^{2}
\end{aligned}
$$



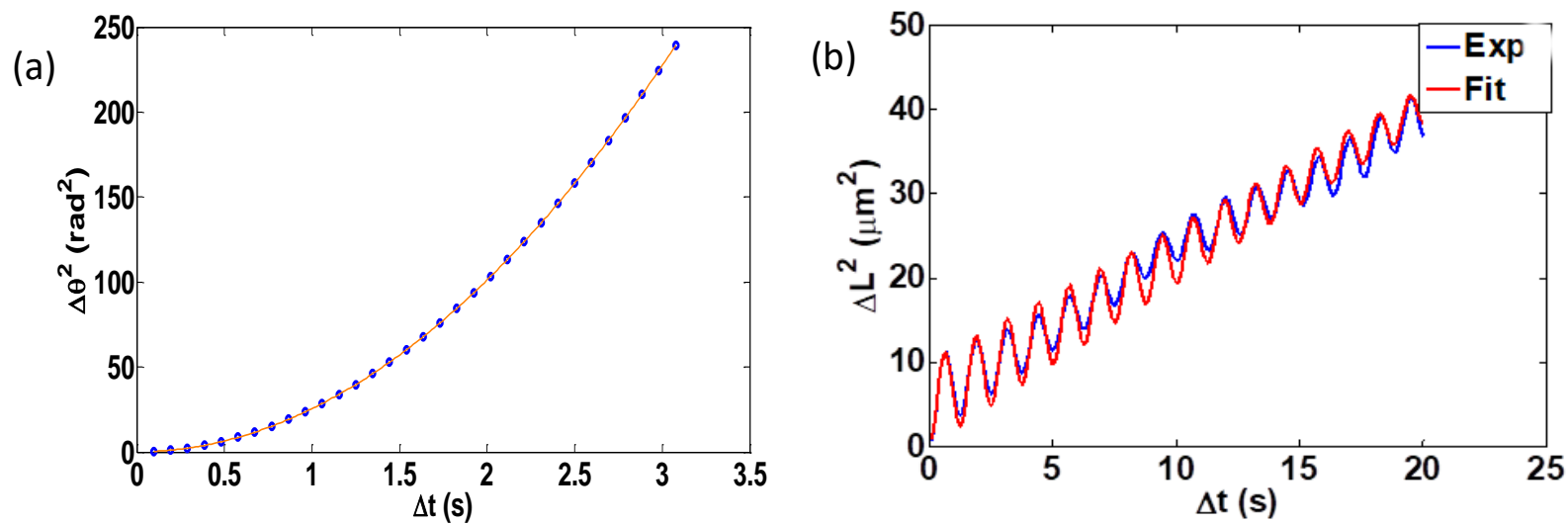

Figure 5.14: Plots of (a) mean-squared angular displacement fitted with Eq. 14 and (b) mean square displacement measurements with analytical fit of Eq. 13.

\begin{tabular}{|l|l|l|l|l|l|}
\hline & $\omega\left(\mathrm{rads}^{-1}\right)$ & $\tau_{R}(s)$ & $\mathrm{D}_{\mathrm{r}}\left(\mathrm{rad}^{2} \mathrm{~s}^{-1}\right)$ & $\mathrm{V}\left(\mu \mathrm{m} \mathrm{s}^{-1}\right)$ & $\mathrm{D}\left(\mu \mathrm{m}^{2} \mathrm{~s}^{-1}\right)$ \\
\hline Analytical MSD fit & 5.02285 & 23.2 & 0.0431 & 8.0 & 0.385 \\
\hline MSAD & 5.02285 & 23.3 & 0.0432 & $\mathrm{n} / \mathrm{a}$ & n/a \\
\hline
\end{tabular}

Table 5.2: Values of $\omega, \tau_{R}, D_{r}, v$, and $D_{t}$ from mean-square displacement fit and mean-square angular displacement measurements.

The trajectory of agglomerate motion in Figure 5.12 (a) shows circular motion with outward petals. The motion trajectory can be viewed as a combination of two circular motions, where a primary circle (with a small radius and angular velocity, $\omega$ ) orbits a secondary circle (with a large radius and angular velocity, $\omega_{s}$ ) in one direction and spins about its center in the same direction.

The circular trajectory above is characterized by a combination of primary and secondary circles. Therefore, identifying the forces acting on the agglomerate to generate the primary and the secondary circles is essential in understanding the circular trajectories. The forces acting on individual particles in agglomerates, with given orientations, can result in continuous centripetal force acting on the agglomerate, which is responsible for the trajectory along the primary circle. The centripetal force is due to the net diffusiophoretic force on the agglomerate directed far from the center of mass. The secondary circle is due to the interaction of gradient fields from the multiple catalytic sites in the agglomerate. The interaction of chemical gradients from multiple catalytic sites results in the secondary circle above. This could be due to the deformation of the concentration gradient, imparting net angular velocity $\omega_{\mathrm{s}}$. 

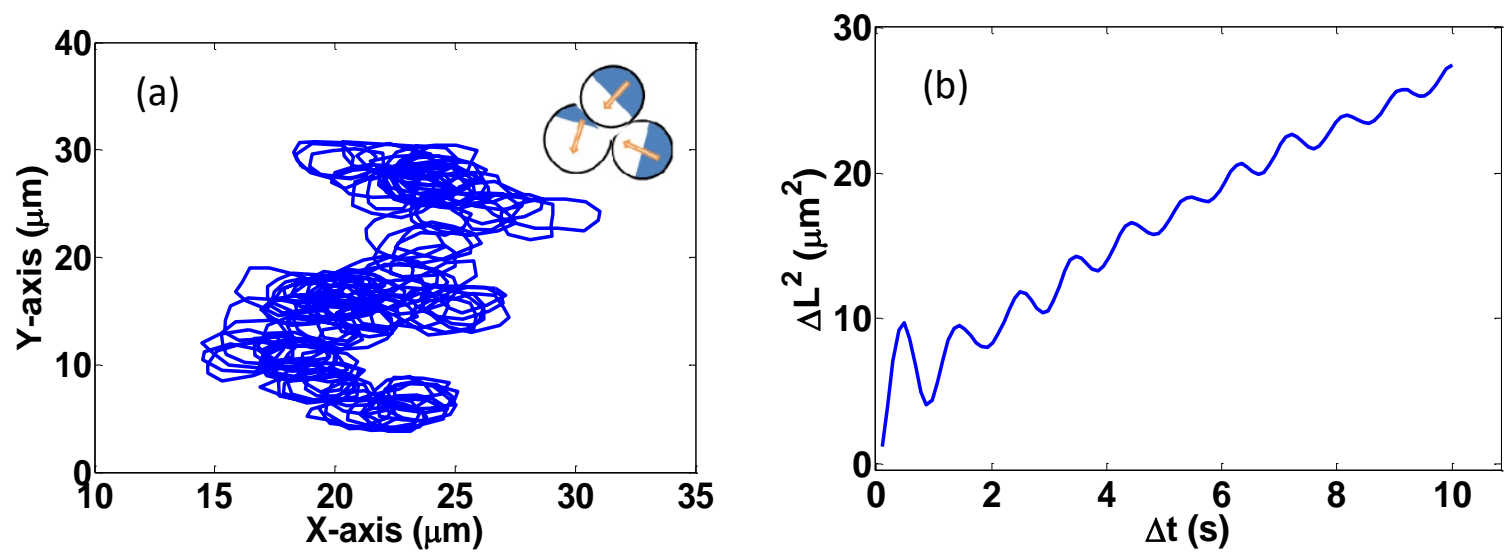

Figure 5.15: Spiral motion of half-coated Pt-silica trimer in $22.5 \% \mathrm{w} / \mathrm{w}$ hydrogen peroxide solution. The orientations of the Pt-silica particles are shown in the insert at (a). The image sequences were collected at $10.40 \mathrm{fps}$. (a) The trajectory of the trimer and (b) the mean square displacement.
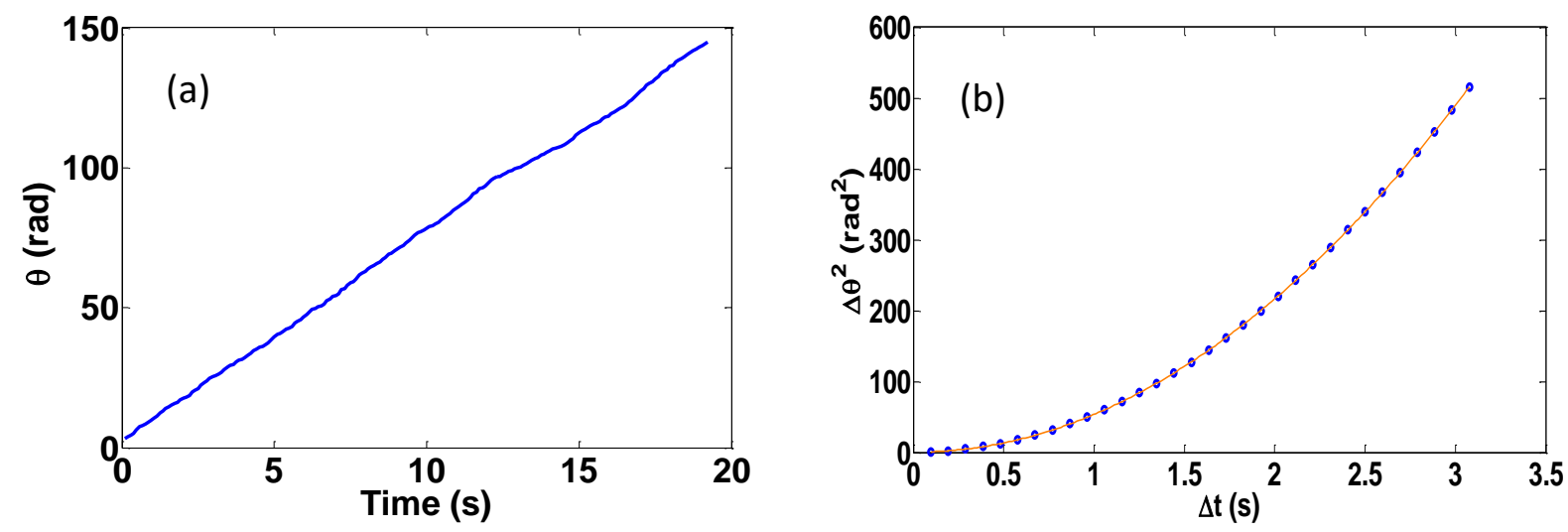

Figure 5.16: (a) Plots of cumulative rotational angle over time and (b) mean square angular displacement.
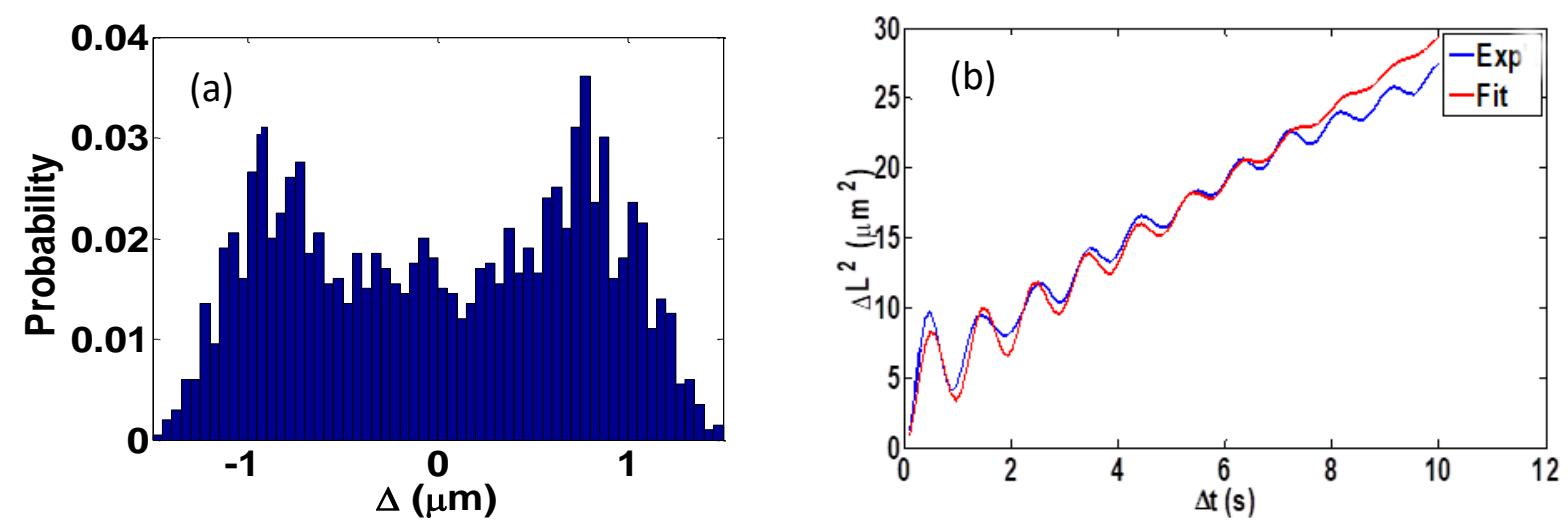

Figure 5.17: (a) Distribution of the step length, $\Delta$, for trimer Pt-silica particles. (b) Mean square displacement measurements with fit using Eq. 13. 


\begin{tabular}{|l|l|l|l|l|l|}
\hline & $\omega\left(\mathrm{rads}^{-1}\right)$ & $\tau_{R}(s)$ & $\mathrm{D}_{\mathrm{r}}\left(\mathrm{rad}^{2} \mathrm{~s}^{-1}\right)$ & $\mathrm{V}\left(\mu \mathrm{m} \mathrm{s}^{-1}\right)$ & $\mathrm{D}\left(\mu m^{2} \mathrm{~s}^{-1}\right)$ \\
\hline Analytical MSD fit & 6.5 & 3.33 & 0.3 & 9.0 & 0.35 \\
\hline MSAD & 7.45 & 3.07 & 0.3257 & $\mathrm{n} / \mathrm{a}$ & n/a \\
\hline
\end{tabular}

Table 5.3: Values of $\omega, \tau_{R}, D_{r}, v$, and $D_{t}$ from mean-square displacement fit and mean-square angular displacement measurements.

The orbiting or spiral motion pattern of these agglomerates is characterized by an oscillating mean square displacement and bimodal step length distribution. Two kinds of forces are responsible for the propulsion of agglomerates with the antisymmetric orientation of the particles. An angular force, which is directly related to the orientation of the particles and is responsible for the primary circular trajectory, while the interaction of the chemical gradients from multiple catalytic sites generates a radial force, which is responsible for a secondary circle with a stable or unstable center.

\subsection{Numerical Calculations}

The fluctuations in translational motions observed in the experiments are thought to be due to a combination of factors: orientation of the catalytic sites of the particles in the agglomerate and strength of the gradient surrounding the particles, which are influenced by Brownian motion. To investigate the behavior of motions observed in the experiments further, we carried out numerical simulations using modified Langevin equations [33] that describe the agglomerate propulsion in two-dimensional space. We focus on the influence of the agglomerate orientations on the motion patterns using dynamic quantities and orientations extracted from the experimental data.

We numerically simulated trajectories of the agglomerates with three catalytic sites by modifying Eqs. 4 with Eqs. 13, as schematically illustrated in Figure 5.18. The trajectories of the agglomerates shown below are a result of various orientated forces

$$
\begin{aligned}
& \frac{d x(t)}{d t}=v \sum_{i=1}^{n} \cos \left(\Theta(t)+\varphi_{i}\right)+\sqrt{2 D_{t}} \xi_{1}(t), \\
& \frac{d y(t)}{d t}=v \sum_{i=1}^{n} \sin \left(\Theta(t)+\varphi_{i}\right)+\sqrt{2 D_{t}} \xi_{2}(t), \\
& \left.\frac{d \Theta(t)}{d t}=\omega+\sqrt{2\left(D_{r}+\frac{\omega_{s}^{2}}{D_{r}}\right.}\right) \xi_{r}(t),
\end{aligned}
$$



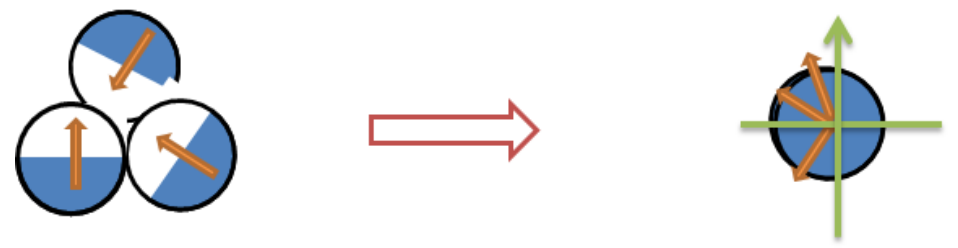

Figure 5.18: Schematic representation of Janus particle agglomerates with multiple catalytic sites oriented in different directions.

Using the dynamics quantities obtained from the experimental results, we simulated the selfpropulsion of the agglomerate with similar orientation geometry to compare the motion.

(a)

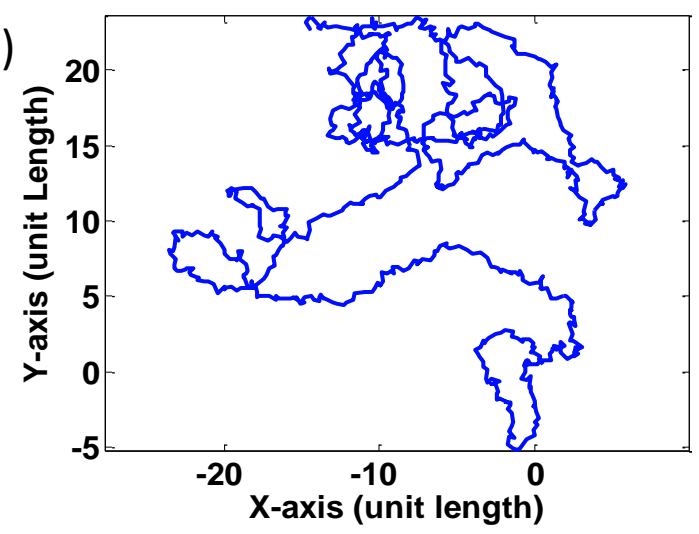

(b)

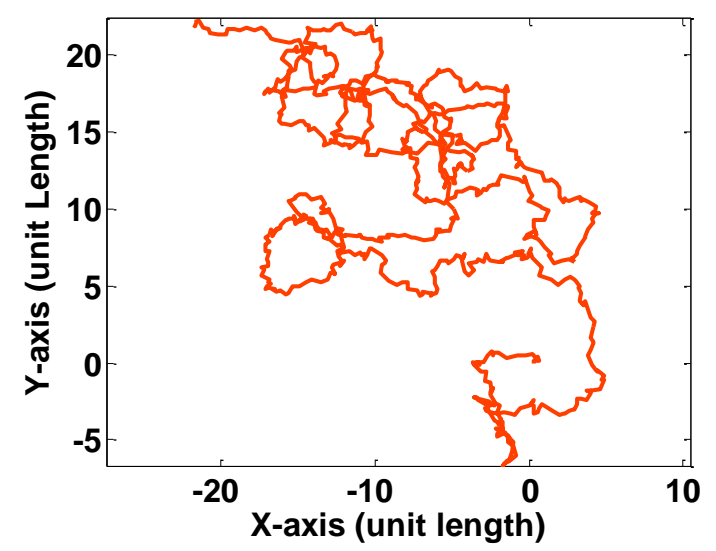

(c)

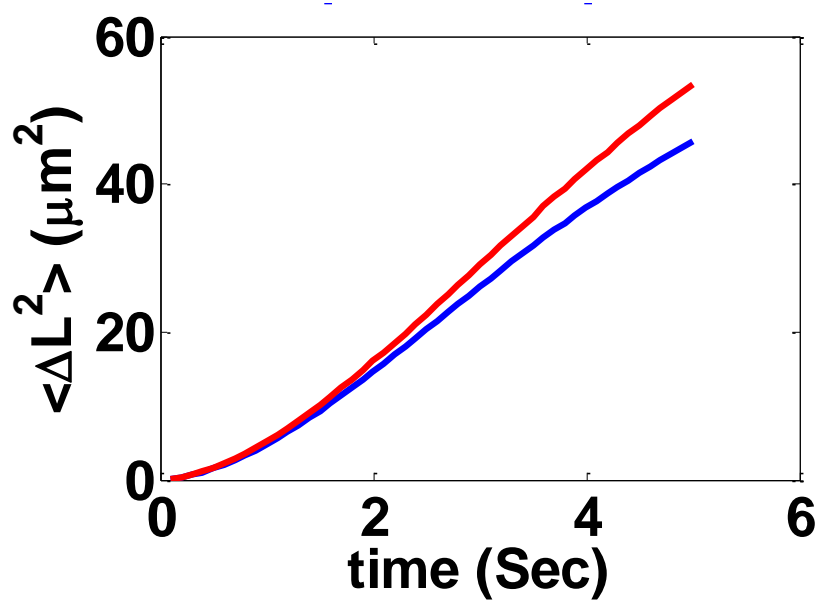

Figure 5.19: Active trimer trajectory with simulation parameters $\varphi_{1}=60^{\circ}, \varphi_{2}=240^{\circ}, \varphi_{3}=90^{\circ}, D_{t}=$ $0.22, D_{r}=0.4, \mathrm{v}=2.10$, and $\omega_{s} \leq \omega=0.5$. (a) - (b) The trajectories of the trimer and (c) mean square displacements. 

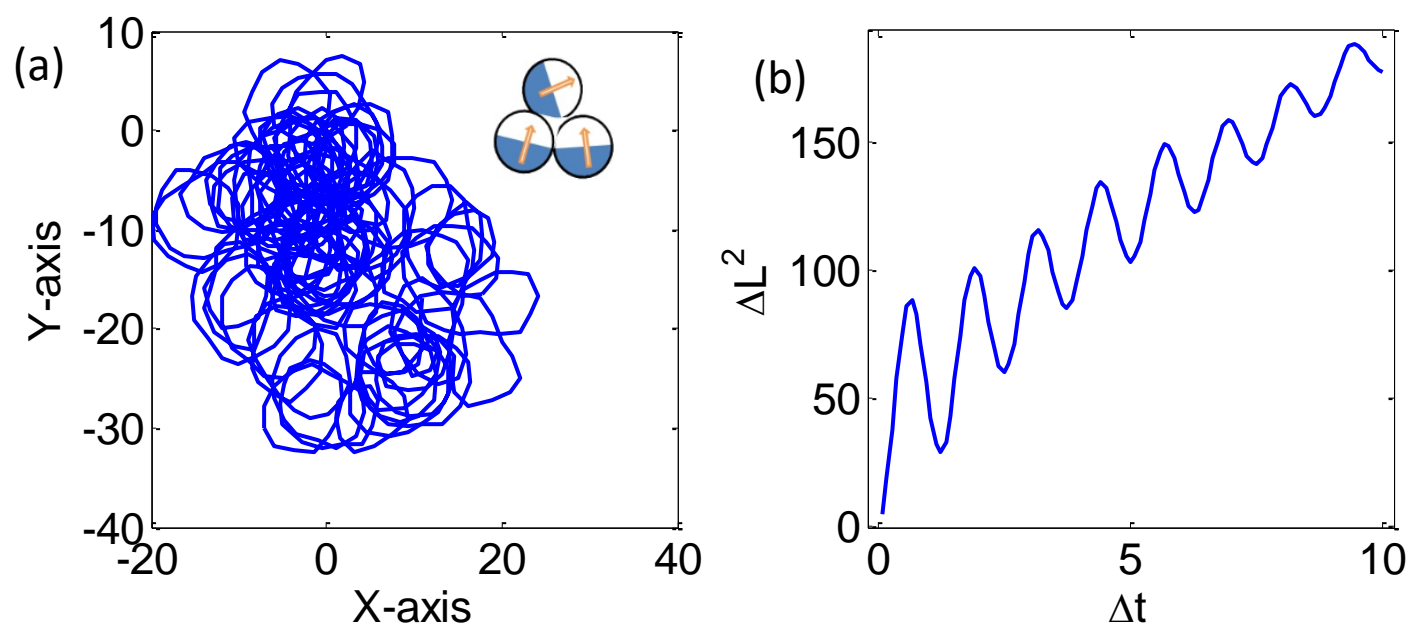

Figure 5.20: Active trimer trajectory with simulation parameters $\varphi_{1}=100^{\circ}, \varphi_{2}=80^{\circ}, \varphi_{3}=60^{\circ}, D_{t}=$ $0.385, D_{r}=0.0432, \mathrm{v}=8.0, \omega_{s}=0.36$, and $\omega=5.0$. (a) The trajectory of the trimer and (b) mean square displacement.
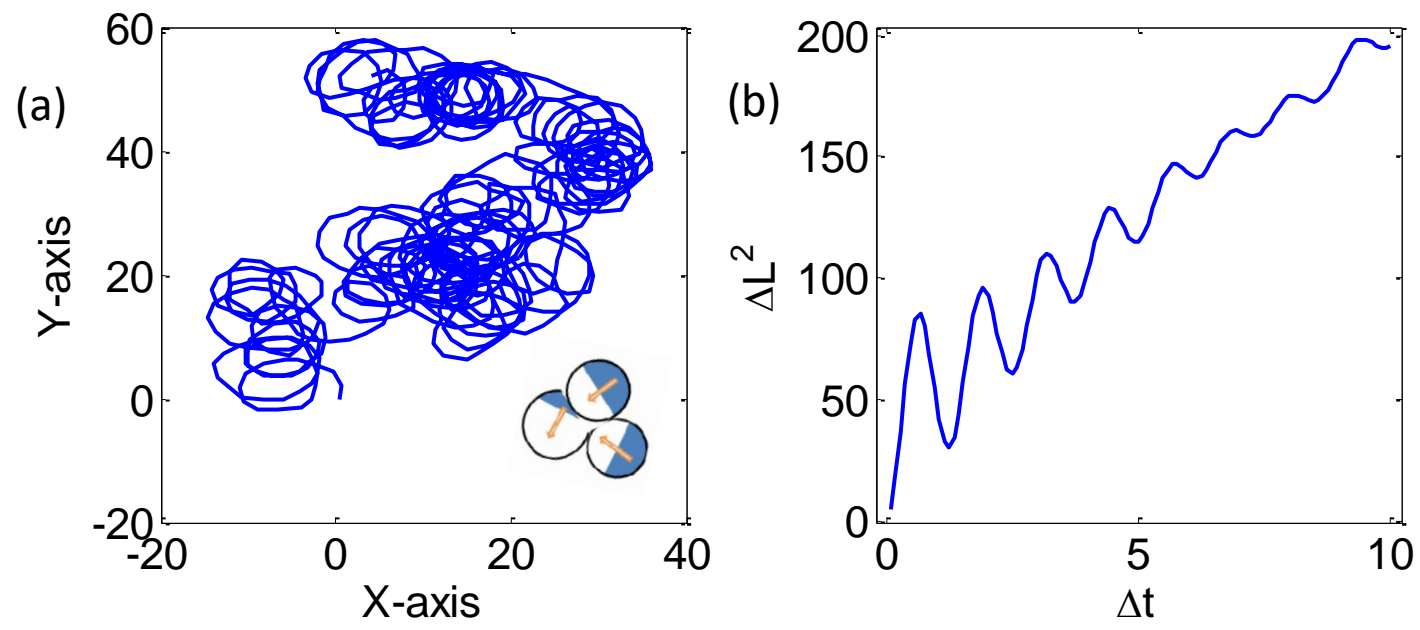

Figure 5.21: Active trimer trajectory with simulation parameters $\varphi_{1}=150^{\circ}, \varphi_{2}=100^{\circ}, \varphi_{3}=260^{\circ}$, $D_{t}=0.35, D_{r}=0.8565, \mathrm{v}=9.5, \omega_{s}=1.56$, and $\omega=4.75$. (a) The trajectory of the trimer and (b) mean square displacement.

The motion patterns vary depending on various oriented forces, $\sum_{i=1}^{n} F_{i}$, and the magnitude of $v$, as shown by the trajectories. The resultant magnitude of the oriented forces occasionally results in a translational motion not directed through the agglomerate center of mass. As a result, a circular motion emerges.

In summary, we have demonstrated experimentally that the resultant motion patterns of the agglomerates depend on the orientation of the particles. We investigated the influence of 
orientation on the motion observed in the experiments by numerical simulations of modified Langevin equations. Analysis of the experimental and model simulations of agglomerates with given particle orientations shows that the motion pattern is as a result of multiple forces acting on the agglomerates. Rotational, orbital, spiral, linear, and Brownian motion are observed experimentally and in the numerical simulations.
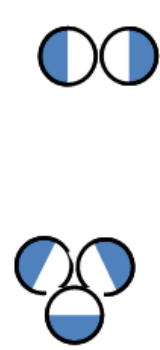
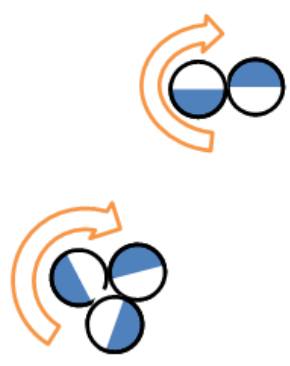
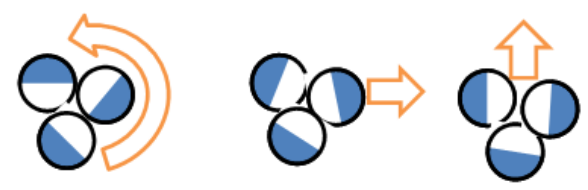

(b)

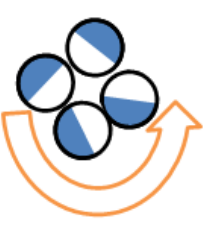

(a)

(c)

Figure 5.22: Illustration of $\mathrm{Pt}_{-} \mathrm{SiO}_{2}$ particle agglomerates with a possible resulting motion for (a) dimers, (b) trimers, and (c) tetramers.

The resultant motion is a result of multiple catalytic sites, which contribute to the forces that propel the particle agglomerates. If the net force does not pass through the center of mass, there will be net torque. The torque results in rotational, orbital or spiral trajectories. The dependence of the motion pattern on the orientation of the particles is demonstrated by the model simulations and analysis. In the absence of net force on the agglomerates, the Brownian motion of the agglomerates is observed.

In this work, we have observed the circular motions of agglomerates in solutions of hydrogen peroxide in a 2D configuration. Two forces are thought to be responsible for the circular trajectory observed. Dynamic quantities are extracted from the measured trajectories 
of motion. The circular motion in the numerical simulations corresponds to the experimental observations.

This study provides useful insights into designing motion of micro motors that optimize motion in a given environment. The experiments and numerical simulations have demonstrated that altering the orientations of the composite particles in agglomerate influences the translational and rotational components of motion. This study provides strategies to design motions of chemically propelled agglomerates with useful functions. The agglomerates, depending on particle orientations, have the capacity to change the pattern of motion depending on the environment. 


\section{References}

[1] S. Ebbens, R. A. L. Jones, A. J. Ryan, R. Golestanian, and J. R. Howse, "Self-assembled autonomous runners and tumblers," Phys. Rev. E 82, 015304 (2010).

[2] H. Ke, S. Ye, L. R. Carroll, and K. Showalter, "Motion Analysis of Self-Propelled Pt-Silica Particles in Hydrogen Peroxide Solution," J. Phys. Chem. A 114, 5462 (2010).

[3] R. Golestanian, T. B. Liverpool, and A. Ajdari, "Propulsion of a Molecular Machine by Asymmetric Distribution of Reaction Products," Phys. Rev. Lett. 94, 220801 (2005).

[4] J. R. Howse, R. A. L. Jones, A. J. Ryan, T. Gough, R. Vafabakhsh, and R. Golestanian, "SelfMotile Colloidal Particles: From Directed Propulsion to Random Walk," Phys. Rev. Lett. 99, 048102 (2007).

[5] R. Golestanian, T. B. Liverpool, and A. Ajdari, "Designing Phoretic Micro- and Nanoswimmers," New J. Phys. 9, 126-1-8 (2007).

[6] M. R. Tinsley, A. J. Steele, and K. Showalter, "Collective Behavior of Particle-Like Chemical Waves," Eur. Phys. J. Special Topics 165, 161-167 (2008).

[7] A. J. Steele, M. Tinsley, and K. Showalter, "Collective Behavior of Stabilized ReactionDiffusion Waves," CHAOS 18, 026108 (2008).

[8] S. J. Ebbens, and J. R. Howse, "In Pursuit of Propulsion at the Nanoscale," Soft Matter 6, 726738 (2010).

[10] P. G. De Gennes, Soft Matter, Nobel Lecture, December 9, 1991.

[11] P. Romanczuk, and L. Schimansky-Geier, "Brownian Motion with Active Fluctuations," Phys. Rev. Lett. 106, 230601 (2011).

[12] L. H. Cisneros, R. Cortez, C. Dombrowski, R. E. Goldstein, and J. O. Kessler, "Fluid Dynamics of Self-propelled Microorganisms, from Individuals to Concentrated Populations," Exp. Fluids. 43, 737-753 (2007).

[13] A. Sokolov, I. S. Aranson, J. O. Kessler, and R. E. Goldstein, "Concentration Dependence of the Collective Dynamics of Swimming Bacteria,"Phy. Rev. 98, 158102 (2007).

[14] A. Snezhko, I. Aranson, and M. Belkin, "Emergent Phenomena in Far-From-Equilibrium Magnetic Granular Ensembles at a Liquid-Air Interface," AIP Conf. Proc. 1145, 143 - 146 (2009). [15] E. M. J. Purcell, “Life at Low Reynolds Number," Phys. 45, 3 (1977). 
[16] S. Vogel, Life in Moving Fluids, Princeton University Press 17, 67 (1994).

[17] M. E. Fisher and A. B. Kolomeisky, "Molecular Motors and the Forces They Exert," Physica A. 274, 241-266 (1999).

[18] J. A. Spudich, “Molecular Motors, Beauty in Complexity," Science 331, 1143 (2011).

[19] A. Walter and A. H. E. Muller, "Janus Particles," Soft Matter 10, 1039 (2008).

[20] M. Wu, J. W. Roberts, S. Kim, D. L. Koch, and M. P. DeLisa, "Collective Bacterial Dynamics Revealed Using a Three-Dimensional Population-Scale Defocused Particle Tracking Technique," Appl. Environ. Microbiol. 72 (7), 4987-4994 (2006).

[21] F. Schweitzer, Brownian Agents and Active Particles, Springer 39 - 46 (2003).

[22] X. Zheng, B. ten Hagen, A. Kaiser, M. Wu, H. Cui, Z. Silber-Li, and H. Lowen, "Non-Gaussian statistics for the motion of self-propelled Janus particles: Experiment versus theory," Phys. Rev. E 88, 032304 (2013).

[23] F. J. Sevilla and M. Sandoval, "Smoluchowski diffusion equation for active Brownian swimmers."

[24] L. F. Valadares, Y. Tao, N. S. Zacharia, V. Kitaev, F. Galembeck, R. Kapral, and G. A. Ozin, "Catalytic nanomotors: Self-propelled sphere dimers," Small 6 (4), 565-572 (2010).

[25] W. Duan, W. Wang, S. Das, V. Yadav, T. E. Mallouk, and A. Sen, "Synthetic nano- and micromachines in analytical chemistry: Sensing, migration, capture, delivery, and separation," Annu. Rev. Anal. Chem. 8, 311-333 (2015).

[26] D. Takagi, A. B. Braunschweig, J. Zhang, and M. J. Shelley, "Dispersion of self-propelled rods undergoing fluctuation-driven flips," Phys. Rev. Lett. 110, 038301 (2013).

[27] G. Volpe, S. Gigan, and G. Volpe, "Simulation of the active Brownian motion of a microswimmer," Am. J. Phys. 82, 659 (2014).

[28] S. Sengupta, M. E. Ibele, and A. Sen, "Fantastic voyage: Designing self-powered nanorobots," Angew. Chem. Int. Ed. 51, 8434-8445 (2012)

[29] W. Wang, W. Duan, S. Ahmed, A. Sen, and T. E. Mallouk, "From one to many: Dynamic assembly and collective behavior of self-propelled colloidal motors," Acc. Chem. Res. 48, 1938-1946 (2015).

[30] C. Maggi, F. Saglimbeni, M. Dipalo, F. D. Angelis, and R. D. Leonardo, "Micromorors with asymmetric shape that efficiently convert light into work by thermocapillary effects," Nat. Commun. 6, 7855 (2015). 
[31] A. Einstein, "On the movement of small particles suspended in stationary liquids required by the molecular-kinetic theory of heat," Annalen der physik 322 (8), 549-560 (1905).

[32] A. Einstein, "Investigations on the theory of the Brownian movement," Dover Publications (1956).

[33] P. Langevin, "On the theory of Brownian motion," C. R. Acad. Sci. (Paris) 146, 530-533 (1908); reviewed by D. S. Lemons and A. Gythiel, Am. J. Phys. 65, 1079 (1997). 


\title{
Synchronization of Coupled Chemical Oscillators and Collective Behavior of Self-Propelled Particles
}

\author{
Desmond Tutu Soti Yengi \\ Dissertation submitted \\ to the Eberly College of Arts and Sciences \\ at West Virginia University
}

in partial fulfillment of the requirements for the degree of

Doctor of Philosophy in

Chemistry

C. Eugene Bennett Department of Chemistry

\section{APPROVAL OF THE EXAMINING COMMITTEE}

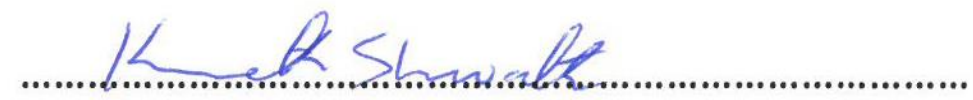

Kenneth/Showalter, Ph،D, Chair

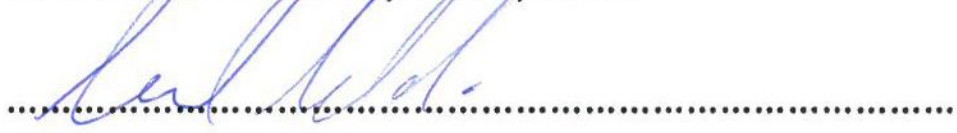

Charles Jaffé, Ph.D.

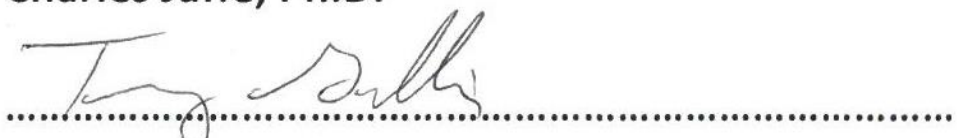

Terry Gullion, Ph.D.
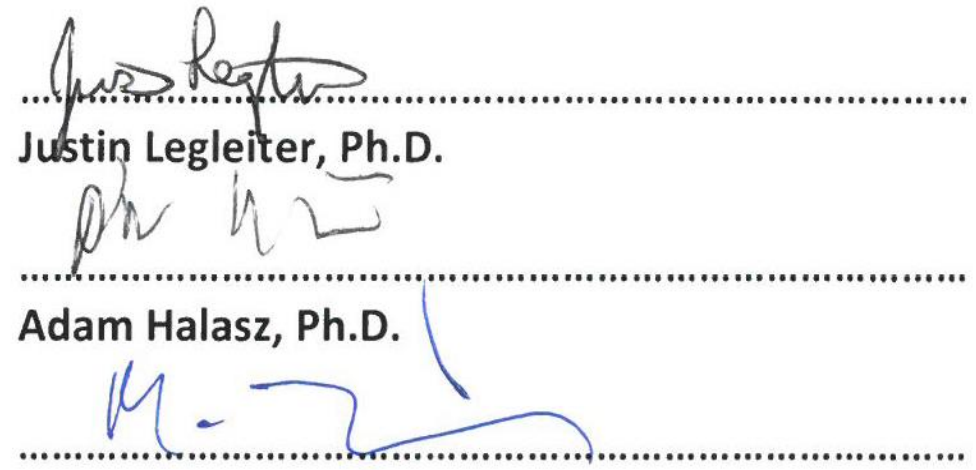

Mark Tinsley, Ph.D.

Date 